\title{
Tuning and Test of Fragmentation Models Based on Identified Particles and Precision Event Shape Data
}

\author{
DELPHI Collaboration
}

\begin{abstract}
Event shape and charged particle inclusive distributions are measured using 750000 decays of the $Z$ to hadrons from the DELPHI detector at LEP. These precise data allow a decisive confrontation with models of the hadronization process. Improved tunings of the JETSET, ARIADNE and HERWIG parton shower models and the JETSET matrix element model are obtained by fitting the models to these DELPHI data as well as to identified particle distributions from all LEP experiments. The description of the data distributions by the models is critically reviewed with special importance attributed to identified particles.
\end{abstract}


P.Abreu ${ }^{21}$, W.Adam ${ }^{50}$, T.Adye ${ }^{37}$, I.Ajinenko ${ }^{42}$, G.D.Alekseev ${ }^{16}$, R.Alemany ${ }^{49}$, P.P.Allport ${ }^{22}$, S.Almehed ${ }^{24}$, U.Amaldi $^{9}$, S.Amato ${ }^{47}$, A.Andreazza ${ }^{28}$, M.L.Andrieux ${ }^{14}$, P.Antilogus ${ }^{9}$, W-D.Apel ${ }^{17}, \quad$ B.Asman ${ }^{44}$, J-E.Augustin ${ }^{25}$, A.Augustinus ${ }^{9}$, P.Baillon ${ }^{9}$, P.Bambade ${ }^{19}$, F.Barao ${ }^{21}$, R.Barate ${ }^{14}$, M.Barbi ${ }^{47}$, D.Y.Bardin ${ }^{16}$, A.Baroncelli $^{40}$, O.Barring ${ }^{24}$, J.A.Barrio ${ }^{26}$, W.Bartl ${ }^{50}$, M.J.Bates ${ }^{37}$, M.Battaglia ${ }^{15}$, M.Baubillier ${ }^{23}$, J.Baudot ${ }^{39}$, K-H.Becks ${ }^{52}$, M.Begalli ${ }^{6}$, P.Beilliere ${ }^{8}$, Yu.Belokopytov ${ }^{9,53}$, K.Belous ${ }^{42}$, A.C.Benvenuti ${ }^{5}$, M.Berggren ${ }^{47}$,

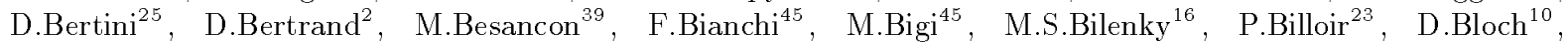
M.Blume $^{52}$, T.Bolognese ${ }^{39}$, M.Bonesini $^{28}$, W.Bonivento ${ }^{28}$, P.S.L.Booth ${ }^{22}$, C.Bosio $^{40}$, O.Botner ${ }^{48}$, E.Boudinov $^{31}$, B.Bouquet ${ }^{19}$, C.Bourdarios ${ }^{9}$, T.J.V.Bowcock ${ }^{22}$, M.Bozzo $^{13}$, P.Branchini ${ }^{40}$, K.D.Brand ${ }^{36}$, T.Brenke $^{52}$, R.A.Brenner ${ }^{15}$, C.Bricman ${ }^{2}$, R.C.A.Brown ${ }^{9}$, P.Bruckman ${ }^{18}$, J-M.Brunet ${ }^{8}$, L.Bugge ${ }^{33}$, T.Buran ${ }^{33}$, T.Burgsmueller ${ }^{52}$, P.Buschmann ${ }^{52}$, A.Buys ${ }^{9}$, S.Cabrera ${ }^{49}$, M.Caccia ${ }^{28}$, M.Calvi ${ }^{28}$, A.J.Camacho Rozas ${ }^{41}$, T.Camporesi ${ }^{9}$, V.Canale ${ }^{38}$, M.Canepa $^{13}$, K.Cankocak ${ }^{44}$, F.Cao ${ }^{2}$, F.Carena ${ }^{9}$, L.Carroll ${ }^{22}$, C.Caso ${ }^{13}$, M.V.Castillo Gimenez ${ }^{49}$, A.Cattai ${ }^{9}$, F.R.Cavallo ${ }^{5}$, V.Chabaud ${ }^{9}$, Ph.Charpentier ${ }^{9}$, L.Chaussard $^{25}$, P.Checchia $^{36}$, G.A.Chelkov ${ }^{16}$, M.Chen ${ }^{2}$, R.Chierici ${ }^{45}$, P.Chliapnikov ${ }^{42}$, P.Chochula ${ }^{7}$, V.Chorowicz ${ }^{9}$, J.Chudoba ${ }^{30}$, V.Cindro ${ }^{43}$, P.Collins $^{9}$, J.L.Contreras ${ }^{19}$, R.Contri ${ }^{13}$, E.Cortina ${ }^{49}$, G.Cosme ${ }^{19}$, F.Cossutti ${ }^{46}$, J-H.Cowell ${ }^{22}$, H.B.Crawley ${ }^{1}$, D.Crennell $^{37}$, G.Crosetti ${ }^{13}$, J.Cuevas Maestro ${ }^{34}$, S.Czellar ${ }^{15}$, E.Dahl-Jensen ${ }^{29}$, J.Dahm ${ }^{52}$, B.Dalmagne ${ }^{19}$, M.Dam $^{29}$, G.Damgaard ${ }^{29}$, P.D.Dauncey ${ }^{37}$, M.Davenport ${ }^{9}$, W.Da Silva ${ }^{23}$, C.Defoix ${ }^{8}$, A.Deghorain ${ }^{2}$, G.Della Ricca ${ }^{46}$, P.Delpierre ${ }^{27}$, N.Demaria ${ }^{35}$, A.De Angelis ${ }^{9}$, W.De Boer ${ }^{17}$, S.De Brabandere ${ }^{2}$, C.De Clercq ${ }^{2}$, C.De La Vaissiere ${ }^{23}$, B.De Lotto ${ }^{46}$, A.De Min $^{36}$, L.De Paula ${ }^{47}$, C.De Saint-Jean ${ }^{39}$, H.Dijkstra ${ }^{9}$, L.Di Ciaccio ${ }^{38}$, A.Di Diodato ${ }^{38}$, F.Djama ${ }^{10}$, J.Dolbeau ${ }^{8}$, M.Donszelmann ${ }^{9}$, K.Doroba ${ }^{51}$, M.Dracos ${ }^{10}$, J.Drees ${ }^{52}$, K.-A.Drees ${ }^{52}$, M.Dris $^{32}$, J-D.Durand ${ }^{25}$, D.Edsall ${ }^{1}$, R.Ehret ${ }^{17}$, G.Eigen ${ }^{4}$, T.Ekelof ${ }^{48}$, G.Ekspong ${ }^{44}$, M.Elsing ${ }^{52}$, J-P.Engel ${ }^{10}$, B.Erzen ${ }^{43}$, M.Espirito Santo ${ }^{21}$,E.Falk ${ }^{24}$, D.Fassouliotis ${ }^{32}$, M.Feindt ${ }^{9}$, A.Ferrer ${ }^{49}$, S.Fichet ${ }^{23}$, T.A.Filippas ${ }^{32}$, A.Firestone $^{1}$, P.-A.Fischer ${ }^{10}$, H.Foeth ${ }^{9}$, E.Fokitis ${ }^{32}$, F.Fontanelli ${ }^{13}$, F.Formenti ${ }^{9}$, B.Franek ${ }^{37}$, P.Frenkiel ${ }^{8}$, D.C.Fries ${ }^{17}$, A.G.Frodesen ${ }^{4}$, R.Fruhwirth ${ }^{50}$, F.Fulda-Quenzer ${ }^{19}$, J.Fuster ${ }^{49}$, A.Galloni ${ }^{22}$, D.Gamba $^{45}$, M.Gandelman ${ }^{6}$, C.Garcia ${ }^{49}$, J.Garcia ${ }^{41}$, C.Gaspar ${ }^{9}$, U.Gasparini ${ }^{36}$, Ph.Gavillet ${ }^{9}$, E.N.Gazis ${ }^{32}$, D.Gele ${ }^{10}$, J-P.Gerber ${ }^{10}$, R.Gokieli ${ }^{51}$, B.Golob ${ }^{43}$, G.Gopal ${ }^{37}$, L.Gorn ${ }^{1}$, M.Gorski ${ }^{51}$, Yu.Gouz ${ }^{45,53}$, V.Gracco ${ }^{13}$, E.Graziani ${ }^{40}$, C.Green ${ }^{22}$, A.Grefrath ${ }^{52}$, P.Gris ${ }^{39}$, G.Grosdidier ${ }^{19}$, K.Grzelak ${ }^{51}$, S.Gumenyuk ${ }^{28,53}$, P.Gunnarsson ${ }^{44}$, M.Gunther ${ }^{48}$, J.Guy ${ }^{37}$, F.Hahn ${ }^{9}$, S.Hahn ${ }^{52}$, Z.Hajduk ${ }^{18}$, A.Hallgren ${ }^{48}$, K.Hamacher ${ }^{52}$, F.J.Harris ${ }^{35}$, V.Hedberg ${ }^{24}$, R.Henriques ${ }^{21}$, J.J.Hernandez ${ }^{49}$, P.Herquet ${ }^{2}$, H.Herr ${ }^{9}$, T.L.Hessing ${ }^{35}$, E.Higon ${ }^{49}$, H.J.Hilke ${ }^{9}$, T.S.Hill ${ }^{1}$, S-O.Holmgren ${ }^{44}$, P.J.Holt ${ }^{35}$, D.Holthuizen ${ }^{31}$, S.Hoorelbeke ${ }^{2}$, M.Houlden ${ }^{22}$, J.Hrubec ${ }^{50}$, K.Huet $^{2}$, K.Hultqvist ${ }^{44}$, J.N.Jackson ${ }^{22}$, R.Jacobsson ${ }^{44}$, P.Jalocha ${ }^{18}$, R.Janik ${ }^{7}$, Ch.Jarlskog ${ }^{24}$, G.Jarlskog ${ }^{24}$, P.Jarry $^{39}$, B.Jean-Marie ${ }^{19}$, E.K.Johansson ${ }^{44}$, L.Jonsson ${ }^{24}$, P.Jonsson ${ }^{24}$, C.Joram ${ }^{9}$, P.Juillot ${ }^{10}$, M.Kaiser $^{17}$, F.Kapusta $^{23}$, K.Karafasoulis ${ }^{11}$, M.Karlsson ${ }^{44}$, E.Karvelas ${ }^{11}$, S.Katsanevas ${ }^{3}$, E.C.Katsoufis ${ }^{32}$, R.Keranen ${ }^{4}$, Yu.Khokhlov $^{42}$, B.A.Khomenko ${ }^{16}$, N.N.Khovanski ${ }^{16}$, B.King ${ }^{22}$, N.J.Kjaer ${ }^{31}$, O.Klapp ${ }^{52}$, H.Klein ${ }^{9}$, A.Klovning ${ }^{4}$, P.Kluit $^{31}$, B.Koene ${ }^{31}$, P.Kokkinias ${ }^{11}$, M.Koratzinos ${ }^{9}$, K.Korcyl ${ }^{18}$, V.Kostioukhine ${ }^{42}$, C.Kourkoumelis ${ }^{3}$, O.Kouznetsov $^{13,16}$, C.Kreuter ${ }^{17}$, I.Kronkvist ${ }^{24}$, Z.Krumstein ${ }^{16}$, W.Krupinski ${ }^{18}$, P.Kubinec ${ }^{7}$, W.Kucewicz ${ }^{18}$, K.Kurvinen $^{15}$, C.Lacasta ${ }^{49}$, I.Laktineh ${ }^{25}$, J.W.Lamsa ${ }^{1}$, L.Lanceri ${ }^{46}$, D.W.Lane ${ }^{1}$, P.Langefeld ${ }^{52}$, V.Lapin ${ }^{42}$, J-P.Laugier $^{39}$, R.Lauhakangas ${ }^{15}$, G.Leder ${ }^{50}$, F.Ledroit ${ }^{14}$, V.Lefebure ${ }^{2}$, C.K.Legan ${ }^{1}$, R.Leitner ${ }^{30}$, J.Lemonne ${ }^{2}$, G.Lenzen $^{52}$, V.Lepeltier ${ }^{19}$, T.Lesiak ${ }^{18}$, J.Libby ${ }^{35}$, D.Liko ${ }^{50}$, R.Lindner ${ }^{52}$, A.Lipniacka $^{44}$, I.Lippi $^{36}$, B.Loerstad $^{24}$, J.G.Loken ${ }^{35}$, J.M.Lopez ${ }^{41}$, D.Loukas ${ }^{11}$, P.Lutz ${ }^{39}$, L.Lyons ${ }^{35}$, J.MacNaughton ${ }^{50}$, G.Maehlum ${ }^{17}$,

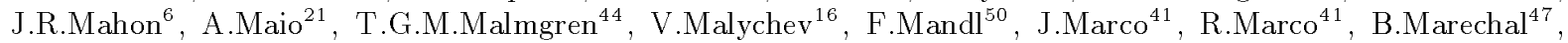
M.Margoni $^{36}$, J-C.Marin ${ }^{9}$, C.Mariotti ${ }^{40}$, A.Markou ${ }^{11}$, C.Martinez-Rivero ${ }^{41}$, F.Martinez-Vidal ${ }^{49}{ }^{\text {, }}$, S.Marti i Garcia ${ }^{22}$, J.Masik ${ }^{30}$, F.Matorras ${ }^{41}$, C.Matteuzzi ${ }^{28}$, G.Matthiae ${ }^{38}$, M.Mazzucato ${ }^{36}$, M.Mc Cubbin ${ }^{9}$, R.Mc Kay ${ }^{1}$, R.Mc Nulty ${ }^{22}$, J.Medbo ${ }^{48}$, M.Merk $^{31}$, C.Meroni $^{28}{ }^{2}$ S.Meyer $^{17}$, W.T.Meyer ${ }^{1}$, A.Miagkov $^{42}$, M.Michelotto $^{36}$, E.Migliore ${ }^{45}$, L.Mirabito ${ }^{25}$, W.A.Mitaroff ${ }^{50}$, U.Mjoernmark ${ }^{24}$, T.Moa ${ }^{44}$, R.Moeller ${ }^{29}$, K.Moenig $^{52}$, M.R.Monge ${ }^{13}$, P.Morettini ${ }^{13}$, H.Mueller ${ }^{17}$, M.Mulders ${ }^{31}$, L.M.Mundim ${ }^{6}$, W.J.Murray ${ }^{37}$, B.Muryn $^{18}$, G.Myatt ${ }^{35}$, F.Naraghi ${ }^{14}$, F.L.Navarria ${ }^{5}$, S.Navas ${ }^{49}$, K.Nawrocki ${ }^{51}$, P.Negri ${ }^{28}$, W.Neumann ${ }^{52}$, N.Neumeister $^{50}$, R.Nicolaidou ${ }^{3}$, B.S.Nielsen ${ }^{29}$, M.Nieuwenhuizen ${ }^{31}$, V.Nikolaenko ${ }^{10}$, P.Niss ${ }^{44}$, A.Nomerotski ${ }^{36}$, A.Normand $^{35}$, W.Oberschulte-Beckmann ${ }^{17}$, V.Obraztsov ${ }^{42}$, A.G.Olshevski ${ }^{16}$, A.Onofre ${ }^{21}$, R.Orava ${ }^{15}$, K.Osterberg $^{15}$, A.Ouraou ${ }^{39}$, P.Paganini ${ }^{19}$, M.Paganoni ${ }^{9,28}$, P.Pages ${ }^{10}$, R.Pain ${ }^{23}$, H.Palka ${ }^{18}$, Th.D.Papadopoulou ${ }^{32}$, K.Papageorgiou ${ }^{11}$, L.Pape ${ }^{9}$, C.Parkes $^{35}$, F.Parodi ${ }^{13}$, A.Passeri ${ }^{40}$, M.Pegoraro $^{36}$, L.Peralta $^{21}$, H.Pernegger ${ }^{50}$, M.Pernicka ${ }^{50}$, A.Perrotta ${ }^{5}$, C.Petridou ${ }^{46}$, A.Petrolini ${ }^{13}$, M.Petrovyck ${ }^{42}$, H.T.Phillips ${ }^{37}$, G.Piana ${ }^{13}$, F.Pierre ${ }^{39}$, S.Plaszczynski ${ }^{19}$, O.Podobrin ${ }^{17}$, M.E.Pol ${ }^{6}$, G.Polok ${ }^{18}$, P.Poropat $^{46}$, V.Pozdniakov $^{16}$, P.Privitera ${ }^{38}$, N.Pukhaeva ${ }^{16}$, A.Pullia ${ }^{28}$, D.Radojicic ${ }^{35}$, S.Ragazzi ${ }^{28}$, H.Rahmani ${ }^{32}$, J.Rames $^{12}$, P.N.Ratoff ${ }^{20}$, A.L.Read ${ }^{33}$, M.Reale ${ }^{52}$, P.Rebecchi ${ }^{19}$, N.G.Redaelli ${ }^{28}$, M.Regler ${ }^{50}$, D.Reid ${ }^{9}$, P.B.Renton ${ }^{35}$, L.K.Resvanis ${ }^{3}$, F.Richard ${ }^{19}$, J.Richardson ${ }^{22}$, J.Ridky ${ }^{12}$, G.Rinaudo ${ }^{45}$, I.Ripp ${ }^{39}$, A.Romero ${ }^{45}$, I.Roncagliolo ${ }^{13}$, P.Ronchese ${ }^{36}$, L.Roos ${ }^{14}$, E.I.Rosenberg ${ }^{1}$, E.Rosso ${ }^{9}, \quad$ P.Roudeau $^{19}$, T.Rovell $^{5}$, W.Ruckstuhl ${ }^{31}$, V.Ruhlmann-Kleider ${ }^{39}$, A.Ruiz ${ }^{41}$, K.Rybicki ${ }^{18}$, H.Saarikko ${ }^{15}$, Y.Sacquin ${ }^{39}$, A.Sadovsky ${ }^{16}$, O.Sahr ${ }^{14}$, G.Sajot $^{14}$, J.Salt $^{49}$, J.Sanchez ${ }^{26}$, M.Sannino ${ }^{13}$, M.Schimmelpfennig ${ }^{17}$, H.Schneider ${ }^{17}$, U.Schwickerath ${ }^{17}$, M.A.E.Schyns ${ }^{52}$,

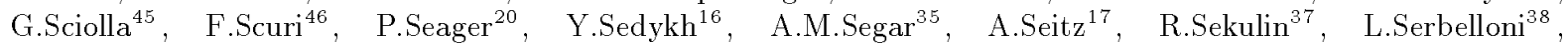
R.C.Shellard ${ }^{6}$, P.Siegrist ${ }^{39}$, R.Silvestre ${ }^{39}$, S.Simonetti ${ }^{39}$, F.Simonetto ${ }^{36}$, A.N.Sisakian ${ }^{16}$, B.Sitar ${ }^{7}$, T.B.Skaali ${ }^{33}$, G.Smadja $^{25}$, N.Smirnov ${ }^{42}$, O.Smirnova ${ }^{24}$, G.R.Smith ${ }^{37}$, A.Sokolov ${ }^{42}$, R.Sosnowski ${ }^{51}$, D.Souza-Santos ${ }^{6}$, T.Spassov ${ }^{21}$, E.Spiriti ${ }^{40}$, P.Sponholz ${ }^{52}$, S.Squarcia ${ }^{13}$, C.Stanescu ${ }^{40}$, S.Stapnes ${ }^{33}$, I.Stavitski ${ }^{36}$, K.Stevenson ${ }^{35}$,

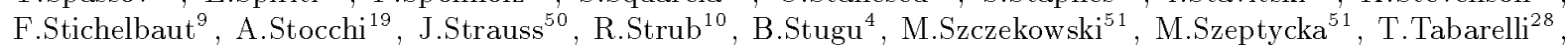


J.P.Tavernet ${ }^{23}$, O.Tchikilev ${ }^{42}$, J.Thomas ${ }^{35}$, A.Tilquin ${ }^{27}$, J.Timmermans ${ }^{31}$, L.G.Tkatchev ${ }^{16}$, T.Todorov ${ }^{10}$, S.Todorova $^{10}$, D.Z.Toet ${ }^{31}$, A.Tomaradze ${ }^{2}$, B.Tome ${ }^{21}$, A.Tonazzo ${ }^{28}$, L.Tortora ${ }^{40}$, G.Transtromer ${ }^{24}$, D.Treille ${ }^{9}$, W.Trischuk ${ }^{9}$, G.Tristram ${ }^{8}$, A.Trombini ${ }^{19}$, C.Troncon ${ }^{28}$, A.Tsirou ${ }^{9}$, M-L.Turluer ${ }^{39}$, I.A.Tyapkin $^{16}$, M.Tyndel $^{37}$, S.Tzamarias $^{22}$, B.Ueberschaer ${ }^{52}$, O.Ullaland ${ }^{9}$, V.Uvarov ${ }^{42}$, G.Valenti ${ }^{5}$, E.Vallazza ${ }^{9}$, G.W.Van Apeldoorn ${ }^{31}$, P.Van Dam ${ }^{31}$, J.Van Eldik ${ }^{31}$, N.Vassilopoulos ${ }^{35}$, G.Vegni ${ }^{28}$, L.Ventura ${ }^{36}$, W.Venus ${ }^{37}$, F.Verbeure ${ }^{2}$, M.Verlato $^{36}$, L.S.Vertogradov ${ }^{16}$, D.Vilanova ${ }^{39}$, P.Vincent ${ }^{25}$, L.Vitale $^{46}$, E.Vlasov ${ }^{42}$, A.S.Vodopyanov ${ }^{16}$, V.Vrba $^{12}$, H.Wahlen $^{52}$, C.Walck ${ }^{44}$, F.Waldner ${ }^{46}$, M.Weierstall ${ }^{52}$, P.Weilhammer ${ }^{9}$, C.Weiser ${ }^{17}$, A.M.Wetherell ${ }^{9}$, D.Wicke ${ }^{52}$, J.H.Wickens ${ }^{2}$, M.Wielers ${ }^{17}$, G.R.Wilkinson ${ }^{35}$, W.S.C.Williams ${ }^{35}$, M.Winter ${ }^{10}$, M.Witek ${ }^{18}$, K.Woschnagg ${ }^{48}$, K.Yip ${ }^{35}$, O.Yushchenko ${ }^{42}$, F.Zach $^{25}$, A.Zaitsev ${ }^{42}$, A.Zalewska ${ }^{9}$, P.Zalewski ${ }^{51}$, D.Zavrtanik ${ }^{43}$, E.Zevgolatakos ${ }^{11}$, N.I.Zimin ${ }^{16}$, M.Zito $^{39}$, D.Zontar ${ }^{43}$, G.C.Zucchelli' ${ }^{44}$, G.Zumerle ${ }^{36}$

\footnotetext{
${ }^{1}$ Department of Physics and Astronomy, Iowa State University, Ames IA 50011-3160, USA

${ }^{2}$ Physics Department, Univ. Instelling Antwerpen, Universiteitsplein 1, B-2610 Wilrijk, Belgium and IIHE, ULB-VUB, Pleinlaan 2, B-1050 Brussels, Belgium

and Faculté des Sciences, Univ. de l'Etat Mons, Av. Maistriau 19, B-7000 Mons, Belgium

${ }^{3}$ Physics Laboratory, University of Athens, Solonos Str. 104, GR-10680 Athens, Greece

${ }^{4}$ Department of Physics, University of Bergen, Allégaten 55, N-5007 Bergen, Norway

${ }^{5}$ Dipartimento di Fisica, Università di Bologna and INFN, Via Irnerio 46, I-40126 Bologna, Italy

${ }^{6}$ Centro Brasileiro de Pesquisas Físicas, rua Xavier Sigaud 150, RJ-22290 Rio de Janeiro, Brazil and Depto. de Física, Pont. Univ. Católica, C.P. 38071 RJ-22453 Rio de Janeiro, Brazil and Inst. de Física, Univ. Estadual do Rio de Janeiro, rua São Francisco Xavier 524, Rio de Janeiro, Brazil ${ }^{7}$ Comenius University, Faculty of Mathematics and Physics, Mlynska Dolina, SK-84215 Bratislava, Slovakia

${ }^{8}$ Collège de France, Lab. de Physique Corpusculaire, IN2P3-CNRS, F-75231 Paris Cedex 05, France

${ }^{9} \mathrm{CERN}, \mathrm{CH}-1211$ Geneva 23, Switzerland

${ }^{10}$ Centre de Recherche Nucléaire, IN2P3 - CNRS/ULP - BP20, F-67037 Strasbourg Cedex, France

${ }^{11}$ Institute of Nuclear Physics, N.C.S.R. Demokritos, P.O. Box 60228, GR-15310 Athens, Greece

${ }^{12}$ FZU, Inst. of Physics of the C.A.S. High Energy Physics Division, Na Slovance 2, 180 40, Praha 8, Czech Republic

${ }^{13}$ Dipartimento di Fisica, Università di Genova and INFN, Via Dodecaneso 33, I-16146 Genova, Italy

${ }^{14}$ Institut des Sciences Nucléaires, IN2P3-CNRS, Université de Grenoble 1, F-38026 Grenoble Cedex, France

${ }^{15}$ Research Institute for High Energy Physics, SEFT, P.O. Box 9, FIN-00014 Helsinki, Finland

${ }^{16}$ Joint Institute for Nuclear Research, Dubna, Head Post Office, P.O. Box 79, 101000 Moscow, Russian Federation

${ }^{17}$ Institut für Experimentelle Kernphysik, Universität Karlsruhe, Postfach 6980, D-76128 Karlsruhe, Germany

${ }^{18}$ Institute of Nuclear Physics and University of Mining and Metalurgy, Ul. Kawiory 26a, PL-30055 Krakow, Poland

${ }^{19}$ Université de Paris-Sud, Lab. de l'Accélérateur Linéaire, IN2P3-CNRS, Bât. 200, F-91405 Orsay Cedex, France

${ }^{20}$ School of Physics and Chemistry, University of Lancaster, Lancaster LA1 4YB, UK

${ }^{21}$ LIP, IST, FCUL - Av. Elias Garcia, 14-1 ${ }^{\circ}$, P-1000 Lisboa Codex, Portugal

${ }^{22}$ Department of Physics, University of Liverpool, P.O. Box 147, Liverpool L69 3BX, UK

${ }^{23}$ LPNHE, IN2P3-CNRS, Universités Paris VI et VII, Tour 33 (RdC), 4 place Jussieu, F-75252 Paris Cedex 05, France

${ }^{24}$ Department of Physics, University of Lund, Sölvegatan 14, S-22363 Lund, Sweden

${ }^{25}$ Université Claude Bernard de Lyon, IPNL, IN2P3-CNRS, F-69622 Villeurbanne Cedex, France

${ }^{26}$ Universidad Complutense, Avda. Complutense s/n, E-28040 Madrid, Spain

${ }^{27}$ Univ. d'Aix - Marseille II - CPP, IN2P3-CNRS, F-13288 Marseille Cedex 09, France

${ }^{28}$ Dipartimento di Fisica, Università di Milano and INFN, Via Celoria 16, I-20133 Milan, Italy

${ }^{29}$ Niels Bohr Institute, Blegdamsvej 17, DK-2100 Copenhagen 0, Denmark

${ }^{30} \mathrm{NC}$, Nuclear Centre of MFF, Charles University, Areal MFF, V Holesovickach 2, 180 00, Praha 8, Czech Republic

${ }^{31}$ NIKHEF, Postbus 41882, NL-1009 DB Amsterdam, The Netherlands

${ }^{32}$ National Technical University, Physics Department, Zografou Campus, GR-15773 Athens, Greece

${ }^{33}$ Physics Department, University of Oslo, Blindern, N-1000 Oslo 3, Norway

${ }^{34}$ Dpto. Fisica, Univ. Oviedo, C/P. Pérez Casas, S/N-33006 Oviedo, Spain

${ }^{35}$ Department of Physics, University of Oxford, Keble Road, Oxford OX1 3RH, UK

${ }^{36}$ Dipartimento di Fisica, Università di Padova and INFN, Via Marzolo 8, I-35131 Padua, Italy

${ }^{37}$ Rutherford Appleton Laboratory, Chilton, Didcot OX11 OQX, UK

${ }^{38}$ Dipartimento di Fisica, Università di Roma II and INFN, Tor Vergata, I-00173 Rome, Italy

${ }^{39} \mathrm{CEA}$, DAPNIA/Service de Physique des Particules, CE-Saclay, F-91191 Gif-sur-Yvette Cedex, France

${ }^{40}$ Istituto Superiore di Sanità, Ist. Naz. di Fisica Nucl. (INFN), Viale Regina Elena 299, I-00161 Rome, Italy

${ }^{41}$ Instituto de Fisica de Cantabria (CSIC-UC), Avda. los Castros, S/N-39006 Santander, Spain, (CICYT-AEN93-0832)

${ }^{42}$ Inst. for High Energy Physics, Serpukov P.O. Box 35, Protvino, (Moscow Region), Russian Federation

${ }^{43} \mathrm{~J}$. Stefan Institute and Department of Physics, University of Ljubljana, Jamova 39, SI-61000 Ljubljana, Slovenia

${ }^{44}$ Fysikum, Stockholm University, Box 6730, S-113 85 Stockholm, Sweden

${ }^{45}$ Dipartimento di Fisica Sperimentale, Università di Torino and INFN, Via P. Giuria 1, I-10125 Turin, Italy

${ }^{46}$ Dipartimento di Fisica, Università di Trieste and INFN, Via A. Valerio 2, I-34127 Trieste, Italy and Istituto di Fisica, Università di Udine, I-33100 Udine, Italy

${ }^{47}$ Univ. Federal do Rio de Janeiro, C.P. 68528 Cidade Univ., Tha do Fundão BR-21945-970 Rio de Janeiro, Brazil

${ }^{48}$ Department of Radiation Sciences, University of Uppsala, P.O. Box 535, S-751 21 Uppsala, Sweden

${ }^{49}$ IFIC, Valencia-CSIC, and D.F.A.M.N., U. de Valencia, Avda. Dr. Moliner 50, E-46100 Burjassot (Valencia), Spain

${ }^{50}$ Institut für Hochenergiephysik, Österr. Akad. d. Wissensch., Nikolsdorfergasse 18, A-1050 Vienna, Austria

${ }^{51}$ Inst. Nuclear Studies and University of Warsaw, Ul. Hoza 69, PL-00681 Warsaw, Poland

${ }^{52}$ Fachbereich Physik, University of Wuppertal, Postfach 100 127, D-42097 Wuppertal, Germany

${ }^{53}$ On leave of absence from IHEP Serpukhov
} 


\section{Introduction}

Precision measurements at LEP using the hadronic final state, such as determinations of the strong coupling constant $\alpha_{s}$ from event shapes, the forward backward asymmetries for quarks, the $Z$ mass and width, or at higher energies the $W^{ \pm}$mass, require a satisfactory model for the properties of the corresponding final states. Perturbative QCD cannot provide full theoretical insight into the transition from primary quarks to observable hadrons, the so-called fragmentation or hadronization process, since only the part of this transition involving large momentum transfer, mainly the radiation of hard gluons or the evolution of a parton shower, is calculable perturbatively. The final formation of hadrons is hidden by the increase of the strong coupling constant $\alpha_{s}$ at small momentum transfer and the ensuing failure of perturbation theory.

Guidance towards a better understanding of the hadronization process must therefore come from detailed experimental investigations of the hadronic final state, including attempts to describe this process by phenomenological models inspired by QCD. LEP I provides a unique and unrivaled opportunity to pursue these studies. The clean welldefined initial state in $e^{+} e^{-}$annihilation provides an excellent testing field, since the event rate at the $Z$ is very high, the energy is large, and the capabilities of the experimental apparatus are much improved with respect to previous experiments.

This paper attempts to determine, for the most frequently used hadronization models, parameters which give an optimal description of a) the observed hadronic event shapes and charged particle inclusive distributions as measured with the DELPHI experiment at LEP, as well as b) the available information on identified particles from all LEP experiments. The latter allows precise determination of more model parameters than the event shapes alone, and also a check of the internal consistency of the models. The performance of these models is compared and critically reviewed.

This paper is organized as follows. Section 2 gives a brief overview of the relevant detector components and describes the experimental procedure applied to determine event shape and inclusive distributions and the related systematic errors. Section 3 discusses the models employed and the relevant parameters. Section 4 describes the optimization strategy applied to obtain the best parameters for the fragmentation models and justifies the choice of the distributions used in the fits: the fits are then discussed in detail and the resulting optimized parameters and their errors are presented. In Section 5, the model predictions are compared with the observed event shape distributions, charge particle spectra, and identified particle data. Finally, Section 6 summarizes.

The appendices contain the definitions of the variables used throughout this paper (Appendix A), followed by tables of the model parameter settings in the Delphi Monte Carlo used to correct the event shape distributions (Appendix B), of the inclusive charged particle and event shape distributions fitted (Appendix $C$ ), of the sensitivities of the fitted parameters to the different distributions used (Appendix D), and of the results of the fits (Appendix E). Finally, Appendix F presents a set of figures comparing the data distributions with the model predictions after the fits.

\section{Detector and Data Analysis}

This analysis uses the 1991, 1992 and 1993 data taken with the DELPHI detector at LEP. The determination of the event shape distributions uses charged particles measured in the solenoidal 1.2 T magnetic field of DELPHI and showers caused by neutral particles 
in the electromagnetic or hadronic calorimeters. The following detectors [1] are relevant to the analysis:

- the Vertex Detector, VD, measuring charged particle coordinates in the plane perpendicular to the beam with up to three layers of silicon micro-strip detectors at radii between $6.3 \mathrm{~cm}$ and $11 \mathrm{~cm}$ and covering polar angles, $\theta$, to the $e^{+}$-beam between $37^{\circ}$ and $143^{\circ}$;

- the Inner Detector, ID, a cylindrical jet chamber with $\theta$ coverage from $17^{\circ}$ to $163^{\circ}$;

- the Time Projection Chamber, TPC, the principal tracking detector of DELPHI, which has 6 sector plates, each with 16 pad rows and 192 sense wires, in the forward and backward hemispheres, inner and outer radii of $30 \mathrm{~cm}$ and $122 \mathrm{~cm}$, and a polar angle coverage from $20^{\circ}$ to $160^{\circ}$;

- the Outer Detector, OD, a five layer drift chamber at $192 \mathrm{~cm}$ radius covering polar angles between $43^{\circ}$ and $137^{\circ}$;

- two sets of forward planar drift chambers, FCA and FCB, with 6 and 12 layers respectively and overall polar angle coverages of $11^{\circ}$ to $35^{\circ}$ and $145^{\circ}$ to $169^{\circ}$;

- the High density Projection Chamber, HPC, a lead-gas electromagnetic calorimeter with a very good spatial resolution located inside the DELPHI coil between $208 \mathrm{~cm}$ and $260 \mathrm{~cm}$ radius and covering polar angles between $43^{\circ}$ and $137^{\circ}$;

- the Forward Electro-Magnetic Calorimeter, FEMC, comprising two lead-glass arrays, one in each endcap, each consisting of 4500 lead glass blocks with a projective geometry, and covering polar angles from $10^{\circ}$ to $36.5^{\circ}$ and from $143.5^{\circ}$ to $170^{\circ}$ to the beam;

- the HAdron Calorimeter, HAC, an iron-gas hadronic calorimeter outside the coil, consisting of at least 19 layers of streamer tubes and $5 \mathrm{~cm}$ thick iron plates also used as flux return, whose overall angular coverage is from $11.2^{\circ}$ to $168.8^{\circ}$.

The performance of the detector is described in [2].

Because the event statistics available for this analysis are very large, the final experimental error is dominated by the systematics. Therefore the selections ensure that the major components of the event were measured in DELPHI with optimal efficiency and resolution, as well as minimizing secondary interactions in the detector material. However, care has been taken not to bias the measured distributions significantly by these cuts.

Charged particles were accepted in the analysis if they satisfied the following criteria:

- momentum $p \geq 200 \mathrm{MeV} / c$,

$-\Delta p / p \leq 1$,

$-20^{\circ} \leq \theta \leq 160^{\circ}$

- measured track length $\geq 50 \mathrm{~cm}$,

- impact parameter with respect to the nominal interaction point within $2 \mathrm{~cm}$ perpendicular to or $5 \mathrm{~cm}$ along the beam.

Furthermore, charged particles with large momenta $(p \geq 25 \mathrm{GeV} / c)$ within the geometrical acceptance of the OD or FCB had to be measured in these detectors as well as in the ID or VD. This requirement ensured a good momentum resolution for high momentum particles.

Energy clusters reconstructed in the calorimeters and not associated with charged particles were accepted as being due to neutral particles (photons or neutral hadrons) if their reconstructed energy exceeded $1 \mathrm{GeV}$ for clusters in the $\mathrm{HAC}$, or $0.5 \mathrm{GeV}$ for clusters in the HPC or FEMC.

Events were selected if: 
- there were at least 5 charged particles selected,

- the total energy of the charged particles exceeded $15 \mathrm{GeV}$, and also exceeded $3 \mathrm{GeV}$ in each half of the detector defined by the plane perpendicular to the beam direction,

- the polar angle of the thrust axis was either between $50^{\circ}$ and $85^{\circ}$, or between $95^{\circ}$ and $130^{\circ}$,

- the momentum imbalance of the event along the beam direction satisfied $\left|\sum p_{z}\right| / \sqrt{s} \leq 0.15$.

About 750000 events were selected. The contamination of beam gas events, $\gamma \gamma$-events, and leptonic events other than $\tau^{+} \tau^{-}$is expected to be less than $0.1 \%$ and has been neglected. The influence of $\tau^{+} \tau^{-}$events, which have a pronounced 2-jet topology and contain high momentum particles, has been determined by a simulation study using events generated by the KORALZ model [3] and treated by the full simulation of the DELPHI detector, DELSIM [2], and the standard data reconstruction chain. The $\tau^{+} \tau^{-}$ contributions have been subtracted from the measured data according to their relative abundance $((0.16 \pm 0.03) \%$ of hadronic events $)$.

Differential inclusive single particle and event shape distributions normalized to the number of hadronic Z decays are presented in Appendix C.1 and C.2 as a function of the physical observables defined in Appendix A. Two different types of result are given: (i) distributions measured from charged particles only and corrected to refer to the part of the final state consisting of charged hadrons only, and (ii) distributions measured from charged plus neutral particles and corrected to refer to the full hadronic final state.

The distributions presented have been corrected for kinematic cuts, limited acceptance and resolution of the detector, and effects due to reinteractions of particles inside the detector material. The correction was calculated using simulated events, generated by JETSET 7.3 PS [4] using the parameter settings given in Appendix B, and treated by the full simulation and analysis chain. For each bin of each distribution, a correction factor $C_{i}$ was calculated as the ratio between the generated and observed distributions. Particles with a proper mean lifetime bigger than $1 \mathrm{~ns}$ were considered as stable particles in the generated distributions. The correction for initial state photon radiation was determined separately, using events generated by JETSET 7.3 PS, with and without initial state radiation as predicted by DYMU3 [5]. The overall correction factors are displayed in the upper insets of the figures presented in Appendix F.

The above simple unfolding by correction factors in general leads to biases of the final results when the detector smearing is bigger than the bin width used, and when the model used to determine the correction factors does not describe the data well [6]. In our case, the model has been tuned to DELPHI data as described in [7] and in this paper, and is in good agreement for all distributions considered. To keep residual biases small with respect to other systematic errors, the bin width of all distributions presented is at least as big as the detector smearing.

To account for possibly imperfect representation of the DELPHI detector or of secondary processes in the simulation program DELSIM, the cuts given above were varied over a wide range, i.e. including smaller polar angles for the event axis, demanding events with more than 7 charged particles, etc. Further cuts were imposed to exclude the boundaries of the TPC sectors for high momentum particles, where the detector effects are known to be less well modelled in the simulation. Systematic uncertainties were deduced as the root-mean-square deviations with respect to the central value. As the systematic error is expected to grow in proportion to the deviation of the overall correction factor from unity, an additional systematic uncertainty, assumed to be $10 \%$ of this deviation, was added quadratically to the above value. A further systematic error was 
added in quadrature for a few bins where the results of the individual data sets corresponding to the different years of data-taking were found to be statistically incompatible. This error was calculated such as to reduce the $\chi^{2}$ per degree of freedom for the merging to unity when it was considered in addition to the statistical errors. As a final step, the systematic uncertainties for each individual variable were smoothed.

The data points and their statistical and systematic errors are given in Tables $11-44$ in Appendix C. The uncertainties shown in the graphs in Appendix F comparing data and models are the final experimental uncertainties used in the fits, obtained by adding the statistical and systematic uncertainties in quadrature. In the fits, they were treated in the same way as purely statistical uncertainties. They are therefore reflected in the statistical errors on the fitted parameters quoted in Appendix E. The systematic errors quoted there were evaluated differently, as described below in section 4.2 , by varying the choice of distributions fitted.

\section{$3 \quad$ Fragmentation Models}

A comprehensive overview of fragmentation models can be found in [8]. This paper considers the most frequently used fragmentation models, namely JETSET 7.3 PS, JETSET 7.4 PS and JETSET 7.4 ME [4], ARIADNE 4.06 [9], and HERWIG $5.8 \mathrm{C}$ [10]. Recently ARIADNE 4.06 has been updated to version 4.08. It has been checked that, with the parameters given below, this new version and version 4.06 predict almost identical shape and inclusive distributions.

HERWIG and JETSET are complete models describing the parton shower evolution or the QCD matrix element calculation, the hadronization of partons into hadrons, and the subsequent decays of short lived particles. ARIADNE models only the parton shower, the subsequent hadronization and decays are treated by JETSET.

\subsection{JETSET 7.3 / 7.4 Parton Shower Model}

JETSET with the parton shower option was used with the switches set as in Table 1. Other switches were left at their default values.

\begin{tabular}{lclccc}
\hline Variable & Value & Variable & Value & Variable & Value \\
\hline MSTJ(11) & 3 & MSTJ(12) & 3 & MSTJ(46) & 3
\end{tabular}

Table 1: Setting of JETSET PS switches used

The JETSET parton shower algorithm is a coherent leading log approximation (LLA) with angular ordering. The shower evolves in the centre of mass frame of the partons, obeying energy and momentum conservation at each step of the shower. In order to represent the 3 -jet cross section correctly at the same time as the 4 -jet and multi-jet cross sections, the lowest order 3 -jet cross-section is reproduced by rejecting some of the first branchings of the initial $q \bar{q}$ system that are predicted by the LLA formalism. Angular ordering of the branchings is explicitly imposed and gluon helicity effects can be included. The value of $\alpha_{s}$ is running, with a scale given by the squared transverse momentum of the branching. The shower evolution is stopped at a mass scale $Q_{0}$, then fragmentation takes over: $Q_{0}$ and $\Lambda_{Q C D}$ (i.e. $\alpha_{s}$ ) are the parameters of the parton shower part of JETSET. 
The fragmentation is performed using the Lund string scheme. This can be formulated as an iterative procedure. A string stretches between the oppositely coloured quark and antiquark via the gluon colour charges. Two gluons nearby in phase space act like a single gluon with equal total momentum, so the string model is infrared safe. The longitudinal momentum fraction $z$ of a hadron is determined using the Lund symmetric fragmentation function:

$$
f(z)=\frac{(1-z)^{a}}{z} \cdot \exp \left(-\frac{b \cdot m_{t}^{2}}{z}\right)
$$

where $m_{t}^{2}=m^{2}+p_{t}^{2}$ is the transverse mass squared of the hadron, and $a$ and $b$ are parameters of the fragmentation function. In principle $b$ is universal, and $a$ can depend on the quark flavour. But for heavy quarks, we use instead the Peterson fragmentation function [11]:

$$
f(z)=\frac{1}{z\left(1-\frac{1}{z}-\frac{\epsilon_{q}}{1-z}\right)^{2}}
$$

with parameter $\epsilon_{q}$ being $\epsilon_{b}$ or $\epsilon_{c}$, since this gives a better description of heavy quark fragmentation. The transverse momenta of the hadrons are determined from the $p_{t}$ values of their constituent quarks, which in turn are chosen from a tunneling process. This leads to a Gaussian $p_{t}^{2}$ behaviour. The relevant parameter is the standard deviation of this distribution, $\sigma_{q}$.

The tunneling also determines the quark flavour generated in the string breakup, leading to a dependence proportional to $\exp \left(-m_{q}^{2}\right)$, and thus to negligible heavy quark production in the fragmentation. Due to the higher mass, even strangeness production is strongly suppressed: $P(u \bar{u}): P(d \bar{d}): P(s \bar{s})=1: 1: \gamma_{s}$, where $\gamma_{s} \approx 0.3$. Mesons are produced according to their quark content in the six multiplets with smallest mass, i.e. in the states: ${ }^{1} S_{0},{ }^{3} S_{1},{ }^{1} P_{1},{ }^{3} P_{0},{ }^{3} P_{1}$ and ${ }^{3} P_{2}$. Contrary to the standard formulation implemented in JETSET, we have defined individual production probabilities $P\left({ }^{2 s+1} L_{j}\right)$ for these multiplets, and also for light, strange, charm and bottom flavours. Except for the light ${ }^{3} P_{0}$ multiplet, the probabilities for the $P$-multiplets are taken to be proportional to $\left(1-P\left({ }^{1} S_{0}\right)-P\left({ }^{3} S_{1}\right)\right) \cdot(2 j+1)$. In a (2 dimensional) string picture, production of particles with non-zero angular momentum (i.e. P-states) is suppressed and expected to be small (about 10\%[12]).

Using additional mass relations, the tunneling mechanism is also applied to baryon production (replacing a quark by a di-quark). Parameters related to baryon production are the relative di-quark production rate $P(q q) / P(q)$, an extra strange di-quark suppression $[P(u s) / P(u d)] / \gamma_{s}$, and an extra suppression $P(q q 1) / P(q q 0)$ of spin 1 di-quarks relative to spin 0 ones leading to $s=3 / 2$ and $s=1 / 2$ baryons. Furthermore, it turned out to be necessary to include an extra suppression of leading baryons, as implemented in JETSET. However, this extra suppression was not used in heavy quark fragmentation, nor in the simulation of heavy particle decays by fragmentation, because the baryon spectra became too soft and there was too strong a suppression of all heavy baryons.

The parameter related to baryon-meson-baryon production, the so-called 'popcorn' parameter, was left at its default value, since experimental determinations of this parameter are not fully conclusive (see [13] and references therein). Furthermore we did not include simulation of Bose Einstein interference. Although, with properly chosen parameters, the Bose Einstein simulation procedure results in a good representation of the correlation functions for identical particles, as well as in a strongly improved description of light meson resonance lineshapes [14,15], the energy momentum rescaling performed in the current procedure (subroutine LUBOEI) is somewhat unphysical, strongly influences 
angular distributions between particles and multi-jet rates, and leads to widely different model parameters, some of which also tend to become unphysical. For example, compare the resulting parameters given in Tables 48 and 49 with the parameters of the DELPHI simulation (Table 10), which includes Bose Einstein interference.

For the tuning of JETSET 7.3 and ARIADNE 4.06, the description of heavy particle decays, and in particular of their branching fractions, has been modified on the basis of recent data. Throughout this paper these modified decays are referred to as 'DELPHI decays'. The modifications are similar to those implemented as default in JETSET 7.4. Similar modifications, based on earlier data, were already implemented in the JETSET 7.3 PS Monte Carlo versions used here for modelling detector effects (Appendix B).

\subsection{JETSET 7.4 Matrix Element Model}

In the historically older matrix element version of JETSET, the parton shower simulation is replaced by the exact second order matrix element calculation which provides up to 4 partons. Two calculations, GKS [16] and ERT [17], are available in JETSET. This paper considers only the default ERT option because it is expected to be more exact [18]. At PETRA/PEP, the predicted 4-jet rate turned out to be too small [18] for a given 3 -jet rate. This has been connected with higher order terms missing in the second order calculation. These terms can be partially accounted for by choosing a suitable scale $\mu$ $\left(Q^{2}=\mu s, \mu \leq 1\right)$ according to the "optimal perturbation theory" description [19]. The ME scale parameter $\mu$ replaces the PS cutoff parameter $Q_{0}$ in the fit, since $Q_{0}$ is not relevant in the ME model. The JETSET 7.4 ME switches used are given in Table 2.

\begin{tabular}{lclclc}
\hline Variable & Value & Variable & Value & Variable & Value \\
\hline MSTJ(11) & 3 & MSTJ(12) & 3 & MSTJ(46) & 3 \\
MSTJ(101) & 2 & MSTJ(111) & 1 & &
\end{tabular}

Table 2: Setting of JETSET 7.4 ME switches used

\subsection{ARIADNE 4.06 Parton Shower Model}

ARIADNE is a particularly elegant formulation of a parton shower based on colour dipoles $[9,20]$. The emission of a gluon from a colour dipole (i.e. the initial quarkantiquark pair) creates two new dipoles, one in between the quark and the gluon and one between the gluon and the antiquark, and each in turn can independently radiate further gluons. It automatically includes ordering in angle (or transverse momentum), as well as azimuthal dependences, in a proper way. The dipole chain resembles the Lund

string. Parameters are the QCD scale parameter $\Lambda_{Q C D}$ and the cut-off scale $p_{t}^{Q C D}$. The latter corresponds to $Q_{0}$ in the JETSET parton shower model. The evolution variable is the transverse momentum squared. As in JETSET, the first order 3-jet cross section is reproduced in ARIADNE. The ARIADNE switches were set as given in Table 3.

\subsection{HERWIG 5.8 C Parton Shower Model}

The evolution of the parton shower in HERWIG is based on the Coherent Parton Branching formalism, an extension of the LLA. It accounts for the leading and subleading logarithmic terms arising from soft and collinear gluon emission. HERWIG pays 


\begin{tabular}{lclclc}
\hline Variable & Value & Variable & Value & Variable & Value \\
\hline MSTA(1) & 1 & MSTA $(3)$ & 0 & MSTA(5) & 1 \\
\hline MSTJ(11) & 3 & MSTJ(12) & 3 & MSTJ(41) & 0 \\
MSTJ(46) & 3 & MSTJ $(105)$ & 0 & &
\end{tabular}

Table 3: Setting of ARIADNE 4.06 switches used

special attention to the simulation of QCD interference phenomena [18]. The most important parameters of the parton shower algorithm are $\Lambda_{Q C D}(\mathrm{QCDLAM})$, the quark masses (RMASS(1-6)), and the effective gluon mass (RMASS(13)), which provides the shower cutoff. The parton shower in HERWIG $5.8 \mathrm{C}$ is matched with the first order 3 -jet cross section. At the end of the parton shower evolution, gluons are split non-perturbatively into $q \bar{q}$-pairs.

The hadronization in HERWIG proceeds via the so-called 'cluster algorithm', based on the preconfinement characteristic of QCD. The colour charge of a parton is compensated to leading order by an anti-colour object which is nearby in phase space. Low mass colour-neutral clusters are formed by combining colour and anti-colour objects. Higher mass clusters are further split into two lighter ones. The splitting is controlled by the parameters CLMAS and CLPOW. In the decay of a cluster containing a quark from the perturbative phase, the direction of this quark is remembered. A Gaussian smearing is applied, controlled by the parameter CLSMR.

Hadrons are then formed in 2-body cluster decays, according to phase space and spin factors. The particle and hadron transverse momenta are thus produced dynamically, as a consequence of the cluster mass spectrum. Particle production in cluster decays can be modified by changing the a priori weights for the individual hadron types. These are VECWT, TENWT ${ }^{2}$ and DECWT for vector or tensor mesons and decuplet baryons respectively, and $\mathrm{PWT}$ for quarks.

Light particle decays are simulated in HERWIG using decay tables. Particles including heavy quarks decay via the decay of the heavy quark and subsequent fragmentation.

Relevant parameter settings used for the tuning of HERWIG are given in Table 4.

\begin{tabular}{lccccc}
\hline Variable & Value & Variable & Value & Variable & Value \\
\hline IPROC & 100 & SUDORD & 1 & CLDIR & 1
\end{tabular}

Table 4: Setting of HERWIG parameters used

\section{Fit of Models to Experimental Data}

Classical optimization strategies, like hill-climbing methods, generally fail to converge if they are applied directly to the optimization of a Monte Carlo model. This is because the physical observables predicted by the model is defined only on a statistical basis (and thus are known only within the statistical errors). Moreover, this straight-forward strategy requires very much computer time, and therefore cannot easily be repeated with changed input data in order to check the influence of systematic errors of the data, etc. Therefore, as in previous work [21-23], the dependence of the physical observables on the

\footnotetext{
${ }^{2}$ The $1^{+-}$and $0^{++}$meson multiplets are not included in HERWIG $5.8 \mathrm{C}$.
} 
model parameters was approximated analytically. For each bin of each distribution, the quadratic approximation

$$
f\left(\vec{p}_{0}+\delta \vec{p}, x\right)=a_{0}^{(0)}(x)+\sum_{i=1}^{n} a_{i}^{(1)}(x) \delta p_{i}+\sum_{i=1}^{n} \sum_{j=i}^{n} a_{i j}^{(2)}(x) \delta p_{i} \delta p_{j} \approx M C\left(\vec{p}_{0}+\delta \vec{p}, x\right)(1)
$$

was used. In this equation, $n$ is the number of parameters to be fitted, and $M C\left(\vec{p}_{0}+\delta \vec{p}, x\right)$ denotes the distribution of a physical observable $x$ predicted for a given set of parameter values $\vec{p}_{0}+\delta \vec{p}$, where $\vec{p}_{0}$ is a central parameter setting and $\delta p_{i}$ is the deviation of parameter $i$ from this setting. The third term in the expansion includes correlation terms between the model parameters. These terms are not present in a linear approximation, such as that used in [23].

The $m=1+n+n(n+1) / 2$ coefficients $a^{(0,1,2)}$ of the expansion were determined by fitting Eq. (1) to $\ell$ reference simulation distributions $(\ell \geq m)$, generated with different parameter settings. This fit is equivalent to solving a system of linear equations:

$$
P \cdot \vec{a}=\overrightarrow{M C}
$$

where $\overrightarrow{M C}$ is the vector of model predictions corresponding to the parameters $\overrightarrow{p_{0}}+\delta \vec{p}^{(k)}$, $\vec{a}$ is the vector of coefficients $a_{i(j)}^{(k)}(x)$, and $P$ is a matrix in which column $k$ contains the parameter variations of model set $k$ :

$$
P_{k, 1 . . m}=\left(1, \quad \delta p_{1}^{(k)}, \ldots, \delta p_{n}^{(k)}, \quad \delta p_{1}^{(k) 2}, \ldots, \delta p_{n}^{(k) 2}, \quad \delta p_{1}^{(k)} \cdot \delta p_{2}^{(k)}, \ldots, \delta p_{n-1}^{(k)} \cdot \delta p_{n}^{(k)}\right)
$$

and $k$ runs from 1 to $\ell$. The optimal solution, for an overconstrained linear system $(\ell>m)$, was obtained using a standard singular value decomposition method [24,25].

The parameters of the $\ell$ reference models (generated with equal statistics) were randomly chosen in parameter space around the central point $\vec{p}_{0}$. Assuming the a priori chosen parameter intervals to be renormalized to \pm 1 , it turns out to be unimportant, except for minor differences in the statistical precision, whether this volume is a hypercube or a hypersphere or whether the points are placed throughout its volume or on its surface. Our choice should ensure that the precision of the fitted linear function is roughly constant within the hypercube.

All simulated sets were generated with equal statistics, large enough that the overall statistical error was small compared with the experimental uncertainty of the data. Therefore the statistical errors of the simulated data sets have been neglected.

The optimum values of the parameters $p_{i}$, their errors $\sigma_{i}$, and their correlation coefficients $\varrho_{i j}$ were then determined from a standard $\chi^{2}$ fit of the analytic approximation (1) to the corresponding data using MINUIT [26]. The fit was done simultaneously for all distributions and all bins considered. Note that the overall analytic approximation contains $n_{\text {bins }} \cdot m$ coefficients $a_{i(j)}^{(0,1,2)}(x)$.

In order to minimise the large number of coefficients to be fitted, if the sensitivity of a distribution to a given model parameter $p_{i}$ was found to be negligible compared with the sensitivity to other parameters (see also next section and Tables 45 - 47 in Appendix D), the dependence of the quadratic expansion (1) on this parameter was suppressed by omitting it from the linear system (2). This led to a better convergence of the minimization and more robust results.

The method was tested by generating $\ell+1=51$ simulated data sets, with 6 important fragmentation parameters, and then simultaneously fitting these 6 parameters to each data set in turn, after using the other 50 data sets to determine the coefficients $a_{i(j)}^{(k)}(x)$. In this case, the statistical error on the coefficients $a_{i(j)}^{(0,1,2)}(x)$ of the quadratic expansion 
(1) was negligible compared with the statistical error of the test set. The pull distributions $\left(p_{i}^{f i t}-p_{i}^{\text {true }}\right) / \sigma_{i}$ were found to be approximately standard normal distributions, showing that the fitting method is self-consistent and unbiased and produces correct errors [14].

\subsection{Choice of Distributions}

Most parameters of a fragmentation model have a well-defined physical meaning. However, some parameters are directly coupled, like $a, b$, and $\sigma_{q}$ in the Lund fragmentation function, while the effect of some parameters on physically observed quantities is obscured by other processes, like decays. Therefore the best choice of distributions for tuning the model parameters is not always evident. Consequently, from the many possible distributions, some have been chosen to determine the central fitted values, and alternative choices have been used to estimate the systematic uncertainties $[7,22,23,27]$.

In practice, to keep the influence of statistical errors as small as possible, it is clear that the models should be fitted to the distributions that show the strongest dependence on the parameter under consideration and least dependence on others. For each distribution $M C(x)$, its sensitivity to a given model parameter, i.e. the quantity:

$$
S_{i}(x)=\left.\frac{\delta M C(x)}{M C(x)}\right|_{p_{i}} /\left.\frac{\delta p_{i}}{p_{i}} \approx \frac{\partial \ln M C(x)}{\partial \ln \left|p_{i}\right|}\right|_{p_{i}}
$$

was therefore calculated, where $\delta M C(x)$ is the change of the distribution $M C(x)$ when the model parameter $p_{i}$ is changed by $\delta p_{i}$ from its central value. Using the fraction $\delta p_{i} / p_{i}$ gives all parameters the same normalization.

Figures 1 and 2 show the sensitivities of the main JETSET PS parameters to some single charged particle and event shape distributions respectively. For a more comprehensive overview, Tables 45 and 46 show the averaged absolute values of the sensitivities to the JETSET 7.4 and HERWIG 5.8 model parameters respectively. However, note that the averaging tends to dilute the sensitivities (compare Fig. 1 with Table 45). The sensitivities for ARIADNE are similar to those for JETSET [14]. For the definitions of the variables, see Appendix A.

The sensitivity to all model parameters tends to be larger for single particle inclusive distributions than for event shape distributions (compare for example Figs. 1 and 2). It can be seen that there is almost no sensitivity to the parton shower cut off $Q_{0}$ in the event shape distributions. For this parameter, the $x_{p}$ spectrum at large $x_{p}$ is the most important. However, correlations among the individual parameters in the inclusive spectra are very strong, as can be seen by the similar (or opposite) behaviour of the sensitivities for different parameters. The opposite, almost symmetric, behaviour of the sensitivities to Lund fragmentation function parameters $a$ and $b$ illustrates the strong correlation between these two parameters, and why it is possible to find good descriptions for many different choices of $a$ and $b$. As expected, $\sigma_{q}$ is best determined by the $p_{t}^{\text {out }}$ spectrum or related quantities. Another important quantity is the charged particle multiplicity, which is known to high precision. Obviously it depends on very many model parameters and also on details of the particle decays.

The differential 3-jet rate as measured by $D_{2}^{\text {Durham }}$ or $D_{2}^{\text {Jade }}$ is best suited to determine $\Lambda_{Q C D}$ because the sensitivities to other parameters are negligible for moderately low values of $y_{c u t}$, see Fig. 2. Thus the determination of $\Lambda_{Q C D}$ can be almost completely decoupled from the determination of other parameters. This also underlines the reliability of $\alpha_{s}$ determinations from differential jet rates. 

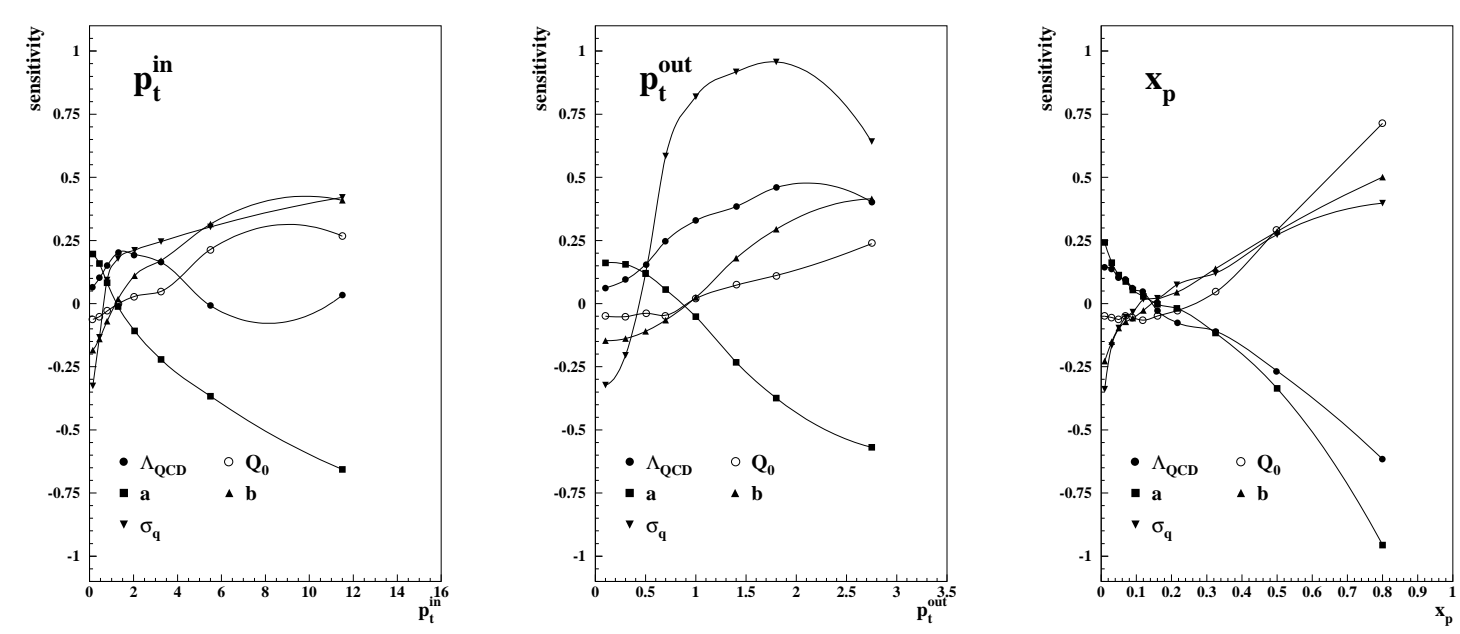

Figure 1: Sensitivity of the JETSET PS parameters $\Lambda_{Q C D}, Q_{0}, a, b$, and $\sigma_{q}$ to the single particle variables $p_{t}^{i n}, p_{t}^{\text {out }}$, and $x_{p}$. The curves are to guide the eye.

Other event shape distributions measuring the overall shape, like the thrust $T$, sphericity $S$, major $M$, or the high hemisphere mass variable $M_{\text {high }}^{2} / E_{v i s}^{2}$, also depend mainly on $\Lambda_{Q C D}$ (see Table 45), except in the 2-jet region (left hand sides of the plots in Fig. 2), where fragmentation effects are also relevant.

Distributions measuring the aplanarity of the events, like the aplanarity $A$, minor $m$, or the lower hemisphere mass variable $M_{\text {low }}^{2} / E_{v i s}^{2}$, tend to show increased sensitivities to $\Lambda_{Q C D}$, but also high sensitivities to other parameters. This illustrates why quantities measuring differences of event shape variables, like the oblateness $O$, hemisphere mass difference $M_{d i f f}^{2} / E_{v i s}^{2}$, and the asymmetry of the energy energy correlation $A E E C$, contrary to widespread belief, depend not only on $\Lambda_{Q C D}$ but also on many other fragmentation parameters.

The above discussion indicates that, for a determination of the general parton-shower and fragmentation parameters, the models should be fitted to:

- the inclusive charged particle distributions as a function of $x_{p}, p_{t}^{\text {in }}$ and $p_{t}^{\text {out }}$ with respect to the thrust or sphericity axis,

- the differential 3-jet rate $D_{3}$, or alternatively $R_{3}$,

- a combination of event shape distributions like $T, M, m$, or $S, A, P$, or $M_{h i g h}^{2} / E_{v i s}^{2}$ and $M_{\text {low }}^{2} / E_{\text {vis }}^{2}$.

Any combination of event shape distributions or event axes for the $p_{t}$ spectra is in principle equivalent. Varying the combination can be used to estimate the stability of the fit and the systematic errors of the parameters determined, or can be viewed as a check of the "predictive power" of the models. However, the strong correlations among the model parameters need to be considered.

The action of the model parameters linked directly to identified particle production (like the strange quark suppression, $\gamma_{s}$, or the relative probability to form a $q q$ pair to make a baryon, $P(q q) / P(q)$, or others) usually follows the physical interpretation more directly. However, identified particle spectra also have high sensitivity to fragmentation parameters (see Tables 45 and 46). Therefore the scheme described above to determine the optimal dependences is used also in this case. 

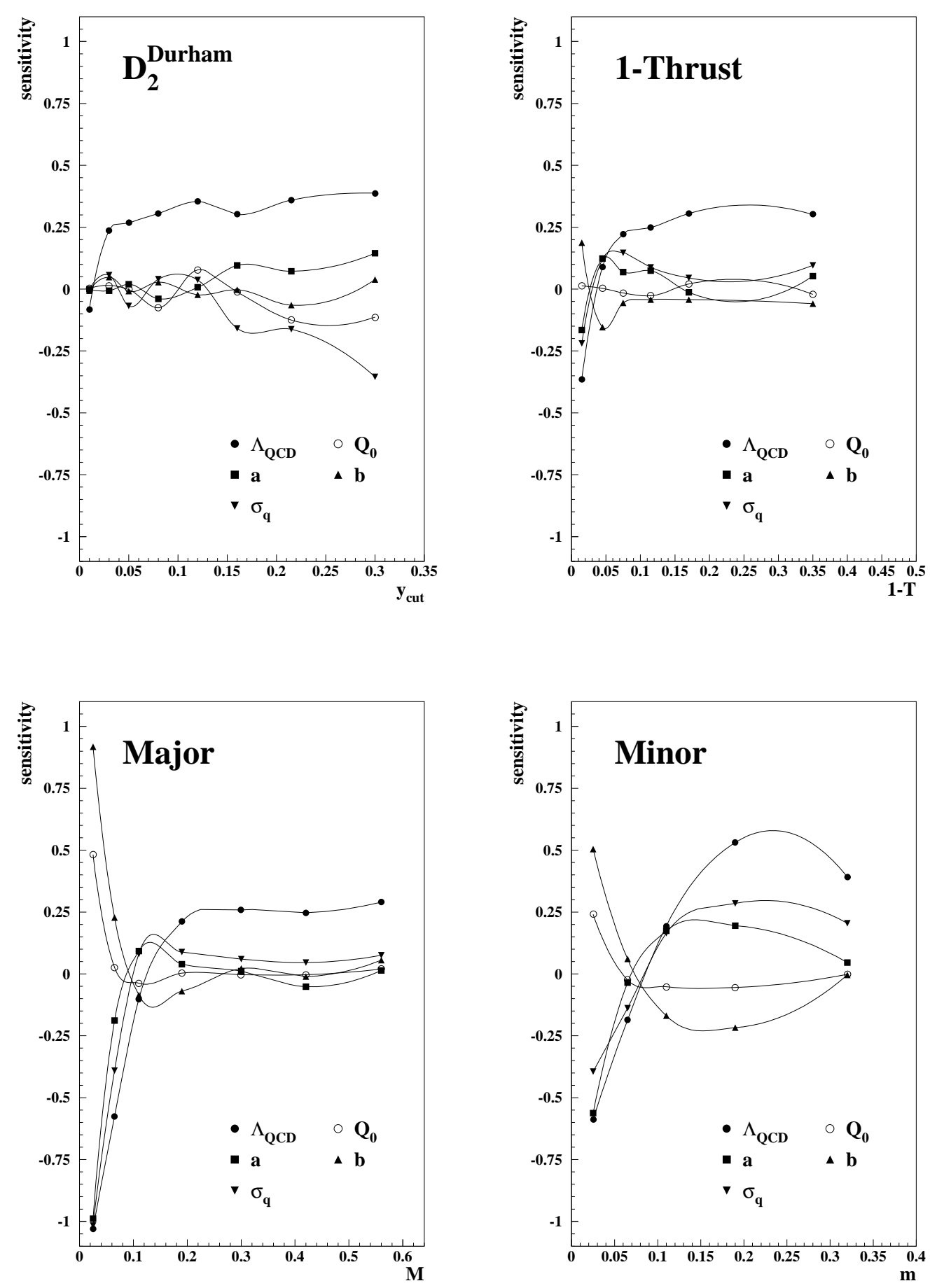

Figure 2: Sensitivity of the JETSET PS parameters $\Lambda_{Q C D}, Q_{0}, a, b$, and $\sigma_{q}$ to the event shape variables $D_{2}^{\text {Durham }}, 1-T, M$, and $m$. The curves are to guide the eye. 


\subsection{Strategy of the Fit}

\subsubsection{JETSET \& ARIADNE}

Fits of the general fragmentation parameters $\left(\Lambda_{Q C D} ; Q_{0}\right.$ or $\mu$ or $p_{t}^{Q C D} ; a, b$ and $\left.\sigma_{q}\right)$ were first performed to the charged particle inclusive distributions and global event shape distributions. In order to determine the coefficients $a^{(0,1,2)}(x)$ in (1), 50 simulated sets with 100000 events each were used for each fit. Parameters related to identified particles were set to values similar to those used for the DELPHI simulation (see Table 10), since these were already known to describe identified particle rates and spectra well.

The average scaled energies $\left\langle x_{E}\right\rangle$ of charm and beauty particles in the models depend only on $\epsilon_{c}, \epsilon_{b}$ and $\Lambda_{Q C D}$. However, the stable hadron spectra depend only weakly on the heavy quark fragmentation parameters. The following procedure was adopted to reduce further the dependences of the stable particle distributions on the heavy quark parameters and at the same time to ensure correct $\left\langle x_{E}\right\rangle$ values for $D^{*}$ and $B$ mesons. The $\left\langle x_{E}\right\rangle$ values for $D^{*}$ and $B$ mesons were determined within the model for several choices of $\Lambda_{Q C D}$ and $\epsilon_{c(b)}$. Then the dependence of $\epsilon_{c(b)}$ on $\left\langle x_{E}\right\rangle_{D^{*}(B)}$ and $\Lambda_{Q C D}$ was parametrized. This allowed the method to choose, for each model set and its given $\Lambda_{Q C D}$ value, corresponding values of $\epsilon_{c(b)}$ such that the model set reproduced within their errors the average heavy meson scaled momenta as measured by the LEP experiments: $\left\langle x_{E}\right\rangle_{D^{*}}=0.504 \pm 0.009,\left\langle x_{E}\right\rangle_{B}=0.701 \pm 0.008[28]$.

The models were then fitted to several combinations of inclusive charged particle and event shape distributions. The results for $\Lambda_{Q C D}$ and $\sigma_{q}$ were relatively stable, those for $Q_{0}$ or $p_{t}^{Q C D}$ less so, and there were many different solutions for $a$ and $b$, because of the strong correlation between these two parameters. Therefore only one central value of $b$ was used in later fits and only $a$ was treated as a variable. The parameter $a$ was preferred because it is less directly coupled to $\sigma_{q}$ in the Lund fragmentation function.

Parameters relevant only to the production of specific particles were then adjusted to the related data. In overview :

- the extra $\eta$ and $\eta^{\prime}$ suppressions were adjusted to data from [29,30];

- the probabilities for producing the different $B$ meson multiplets were adjusted to agree with recent measurements $[31,32]$ and the corresponding $D$ meson probabilities were interpolated between the $B$ and light meson values;

- $[P(u s) / P(u d)] / \gamma_{s}$, the strange baryon suppression, was adjusted to the ratio of $\Lambda^{0}$ to proton production [13,33-36];

- the leading baryon suppression was adjusted to the high momentum tail of the proton and $\Lambda^{0}$ spectra $[13,33-36]$;

- $P(q q 1) / P(q q 0)$, the spin 1 di-quark suppression, was adjusted to the ratio of $\Sigma(1385)$ to $\Lambda^{0}$ or proton production $[13,33,34,36,37]$.

Then a simultaneous fit of 10 important parameters (see Tables 48 - 52 in Appendix E) was prepared, by generating 100 simulated data sets of 100000 events each. The analytical approximation obtained from these simulated data sets was then fitted to various choices of inclusive charged particle, event shape, and identified particle distributions.

The inclusive charged particle and event shape distributions measured by DELPHI in this analysis were used for these fits in various combinations, as shown in Table 5. The mean charged particle multiplicity at the $Z$ used in the fits, $\left\langle N_{c h}\right\rangle=20.92 \pm 0.24$, was an average of all available results [38].

The identified particle distributions used included results from all LEP experiments. The combinations chosen are shown in Table 6 . They were selected to take into ac- 
count the discrepancies observed for the proton data from different experiments and the imperfect representation of the $K$ spectra in the models.

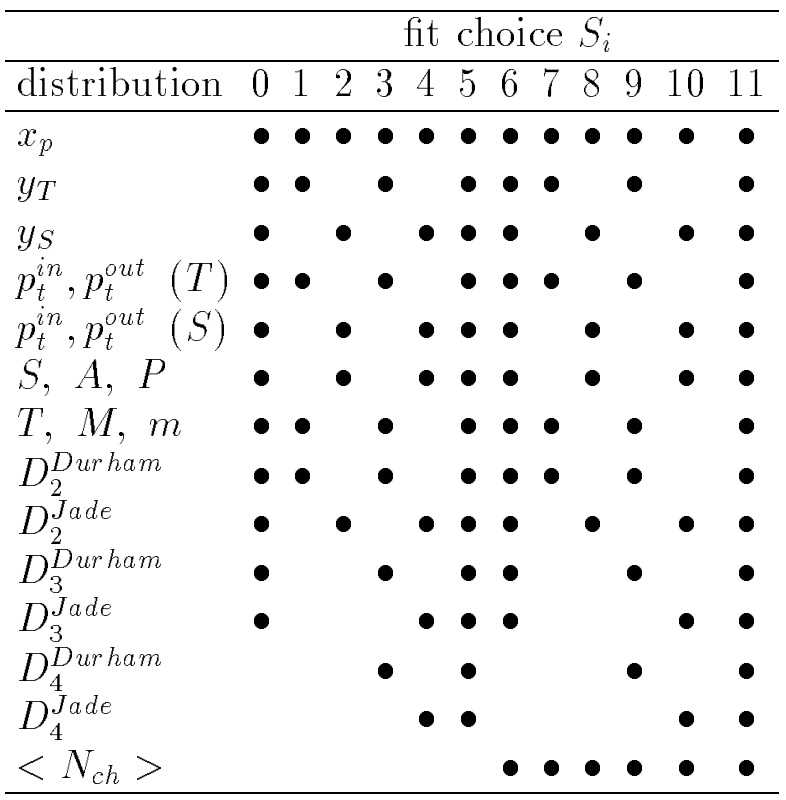

Table 5: Overview of the combinations of inclusive charged particle and event shape distributions used for the fits of JETSET, ARIADNE and HERWIG. Distributions used are marked by $\bullet$. The central results presented correspond to the choice $S_{6}$. The other combinations were used to provide estimates of the systematic errors.

\begin{tabular}{|c|c|c|}
\hline & & fit choice $P_{i}$ \\
\hline data & & $\begin{array}{lllll}0 & 1 & 2 & 3 & 4\end{array}$ \\
\hline$\rho^{\circ}$ & DELPHI [39] & $\bullet \bullet$ \\
\hline$\omega$ & L3 $[40]$ & • \\
\hline$f_{0}, f_{2}$ & DELPHI [39] & - • \\
\hline$K^{0}$ & ALEPH [13], OPAL [37] & $\bullet$ \\
\hline$K^{ \pm}$ & ALEPH [33], DELPHI [34], OPAL [36] & $\bullet$ \\
\hline$K^{* 0}$ & OPAL [41] & $\bullet$ \\
\hline$K^{* \pm}$ & ALEPH [42], DELPHI [39], OPAL [41] & $\bullet$ \\
\hline$\Phi$ & DELPHI [34], OPAL [36] & $\bullet$ \\
\hline$p$ & ALEPH [33], DELPHI [34] & $\bullet \bullet$ \\
\hline$p$ & OPAL $[36]$ & $\bullet \bullet$ \\
\hline
\end{tabular}

Table 6: Overview of the combinations of identified particle data used for the JETSET and ARIADNE fits. Data used are marked by $\bullet$. The central result presented corresponds to the choice $P_{0}$. The other combinations were used to provide estimates of the systematic errors.

The proton data were used in the fit only to determine the di-quark suppression parameter $P(q q) / P(q)$ and were excluded from the $\chi^{2}$ calculation for the fits of the remaining parameters. Baryons at intermediate and large momenta are likely, according to the models, to be primary produced particles. Therefore these data strongly influence 
the primary fragmentation function and the related parameters. Since some experimental discrepancies are present in the proton spectra, and because it had proved necessary to modify the fragmentation function by an extra suppression at large momenta, this strong impact on the fit results was considered to be unphysical and therefore was excluded. The $\Lambda^{0}$ data were also excluded from the final fits for similar reasons, and because they are not described well enough by the models.

Separate fits were performed for all possible combinations of the 12 choices of inclusive and event shape distributions in Table 5 with the 5 possible choices of identified particle data in Table 6. A full MINOS [26] error estimate was performed for all parameters.

To check the stability of the fits, the optimization was started with 6 random start values of the fragmentation parameters. The fits were stable and converged to the same solution in $95 \%$ of cases. The differences occurred mainly for two strongly correlated parameters. Where the fits gave more than 1 solution, the one with the better $\chi^{2}$ was kept.

The central results presented correspond to the combination $S_{6} P_{0}$. This combination was chosen because it contains event shape distributions linear and quadratic in the particle momenta, the charged particle multiplicity, and all relevant identified particle information, so it should result in a complete overall description. The other combinations were used to provide estimates of the systematic errors. The results are given, with their statistical and systematic errors, in Appendix E, Tables 48 - 52.

The results for some of the parameters are strongly correlated. Typical correlation coefficients for JETSET 7.4 (default decays) and ARIADNE 4.06 (DELPHI decays) are given in Appendix E, Tables 54 and 55. Besides these statistical correlations, further correlations exist due to the different possible choices of input data. The upward (downward) systematic error quoted is the root-mean-square spread of the results obtained from all combinations of input data that gave parameter values bigger (smaller) than the central fit.

The following observations were made by comparing the results of the different input data choices for the JETSET PS fits.

As should be expected, the results for $\Lambda_{Q C D}, Q_{0}$ and $\sigma_{q}$ were almost independent of the choice of identified particle data. If just $D_{2}$ was included in the fit, the resulting value of $\Lambda_{Q C D}$ did not depend on the algorithm used (JADE or DURHAM). However, if the higher jet rates $\left(D_{3}, D_{4}\right)$ were included, the $\Lambda_{Q C D}$ value was somewhat bigger $(\approx 8 \%)$ using the JADE algorithm. The values of $\Lambda_{Q C D}$ and $Q_{0}$ were positively correlated, implying that the number of final partons is more stable within the models than might be expected from the error of $Q_{0}$ alone. The parameters $\Lambda_{Q C D}, a$ and $\sigma_{q}$ were anticorrelated, due to a compensation of the transverse momenta generated in the parton shower and fragmentation phases of the model.

The results for $\gamma_{s}$ were higher if the $K^{ \pm}$data were included as well as the $K^{0}$ data. $P(q q) / P(q)$ was larger for the ALEPH proton spectrum than for the OPAL one. Production parameters for strange vector and pseudoscalar mesons were anticorrelated. If the charged particle multiplicity was not included, and therefore the predicted multiplicity was smaller than the measured result, the primary production probabilities for light pseudoscalar and vector mesons were $P\left({ }^{1} S_{0}\right)_{u d} \approx 0.40$ and $P\left({ }^{3} S_{1}\right)_{u d} \approx 0.26$ respectively. If the multiplicity was fixed to $N_{c h} \approx 20.9$, these values were 0.28 and 0.29 . This implies substantial production probabilities for light p-wave mesons of 0.36 to 0.43 .

Most of these observations also apply to ARIADNE. However, in this case the different choices of $D_{2}, D_{3}$, etc. led to stable results for $\Lambda_{Q C D}$. There was a tendency to obtain slightly bigger values for $\Lambda_{Q C D}$ from the JADE algorithm (0.245) than from the Durham 
one $(0.237)$. This is again consistent with the bigger values for $p_{t}^{Q C D}$ obtained from JADE $(\approx 0.9 \mathrm{GeV} / \mathrm{c})$ than from DURHAM $(\approx 0.6 \mathrm{GeV} / \mathrm{c})$.

For JETSET ME, $\Lambda_{Q C D}$ was found to be anticorrelated with the scale $\mu$. The fragmentation parameter values differ from those from JETSET PS. Especially $\sigma_{q} \approx 0.48$ $\mathrm{GeV} / \mathrm{c}$ is much bigger. This partially compensates the missing higher orders in the ME model. The values of $\gamma_{s}$ and $P(q q) / P(q)$ are smaller than for the PS case and depend less on the choice of input data.

\subsubsection{HERWIG}

HERWIG uses fewer parameters than JETSET, especially in the hadronization sector of the model. Therefore a simultaneous fit of all model parameters which are found to be important is easily performed. These parameters (see Table 46) are:

- the QCD scale parameter QCDLAM and the gluon mass RMASS(13) as major parameters of the parton shower phase,

- the cluster fragmentation parameters CLMAX, CLPOW, and CLSMR (CLDIR=1),

- the a priori weights PWT(3) for strange quarks, PWT(7) for di-quarks, and DECWT for decuplet baryons.

Besides the particle spectra, the cluster parameters also strongly influence the stable charged particle and event shape distributions. Fits were therefore performed to combinations of inclusive charged particle and event shape distributions (see Table 5) and identified particle data (see Table 7). The average scaled momenta of heavy mesons are important for the determination of CLMAX and CLPOW.

The identified particle data sets were again chosen (see Table 7) such that systematic differences in the data would be reflected in the systematic errors of the fitted parameters. The central fit result for HERWIG (see Table 53) corresponds to the combination $S_{6} P_{5}$. The other combinations were used to provide estimates of the systematic errors.

For HERWIG, comparing the results of the different input data choices showed that the values obtained for QCDLAM were in general bigger (by $\approx 0.01$ ) when fitting DURHAM rather than JADE jet rates. Contrary to the JETSET case, QCDLAM was smaller (by $\approx 0.005$ ) when the differential 2-, 3-, and 4-jet rates were all fitted, rather than the 2-jet rate only. The gluon mass RMASS(13) showed some dependence on the identified particle information selected, and on the charged particle multiplicity. The cluster parameters CLMAX and CLPOW depended on the identified particle spectra only when the multiplicity was not included in the fit. CLPOW was higher for JADE than for DURHAM jet rates. CLSMR depended only on the inclusive charged particle and event shape information. Including the baryon decuplet data tended to spoil the description of the octet sector.

\section{Comparison of Models to Data}

The fits of the individual fragmentation models are compared with corrected DELPHI data (this analysis) in Figs. 3-38 collected in Appendix F. Figs. 3-32 and 37-38 show data measured from charged particles only, while Figs. 33-36 compare the models with a few selected distributions measured for charged and neutral particles. All distributions are corrected to the corresponding final states. The lower insets of the plots depict the relative deviation of the models from the data. Also shown, as shaded areas in these 


\begin{tabular}{|c|c|c|}
\hline & & fit choice $P_{i}$ \\
\hline data & & $\begin{array}{llllllllll}0 & 1 & 2 & 3 & 4 & 5 & 6 & 7 & 8 & 9\end{array}$ \\
\hline$\rho^{\circ}$ & DELPHI [39] & $\bullet \bullet \bullet \bullet \bullet \bullet \bullet \bullet \bullet \bullet$ \\
\hline$\omega$ & L3 $[40]$ & $\bullet \bullet \bullet \bullet \bullet \bullet \bullet \bullet \bullet \bullet$ \\
\hline$f_{2}$ & DELPHI [39] & $\bullet \bullet \bullet \bullet \bullet \bullet \bullet \bullet \bullet \bullet$ \\
\hline$K^{0}$ & ALEPH [13], OPAL [37] & $\bullet \bullet \bullet \bullet \bullet \bullet \bullet \bullet \bullet \bullet$ \\
\hline$K^{ \pm}$ & ALEPH [33], DELPHI [34], OPAL [36] & $\bullet \bullet \bullet$ \\
\hline$K^{* 0}$ & OPAL $[36]$ & $\bullet \bullet \bullet \bullet \bullet \bullet \bullet \bullet \bullet \bullet$ \\
\hline$K^{* \pm}$ & ALEPH [42], DELPHI [39], OPAL [41] & $\bullet \bullet \bullet \bullet \bullet \bullet \bullet \bullet \bullet \bullet$ \\
\hline$\Phi$ & DELPHI [34], OPAL [36] & $\bullet \bullet \bullet \bullet \bullet \bullet \bullet \bullet \bullet \bullet$ \\
\hline$\Lambda^{0}$ & ALEPH [13], DELPHI [35] & $\bullet \bullet$ \\
\hline$\Lambda^{0}$ & OPAL $[41]$ & $\bullet \bullet$ \\
\hline$p$ & ALEPH [33], DELPHI [34] & $\bullet \bullet \bullet$ \\
\hline$p$ & OPAL $[36]$ & $\bullet \bullet \bullet$ \\
\hline$\eta$ & ALEPH $[30]$ & $\bullet \bullet \bullet \bullet \bullet \bullet \bullet \bullet \bullet \bullet$ \\
\hline$\eta$ & ALEPH $[30]$ & $\bullet \bullet \bullet \bullet \bullet \bullet \bullet \bullet \bullet \bullet$ \\
\hline$<x_{E}>D^{* \pm}, D^{* 0}$ & LEP average [28] & $\bullet \bullet \bullet \bullet \bullet \bullet \bullet \bullet \bullet \bullet$ \\
\hline$<x_{E}>B^{0}, B^{ \pm}$ & LEP average [28] & $\bullet \bullet \bullet \bullet \bullet \bullet \bullet \bullet \bullet \bullet$ \\
\hline$\Sigma^{ \pm}(1385)$ & DELPHI [35], OPAL [41] & $\bullet \bullet \bullet \bullet \bullet$ \\
\hline$\Xi^{-}$ & DELPHI [35], OPAL [41] & $\bullet \bullet \bullet \bullet$ \\
\hline$\Xi_{0}(1530)$ & DELPHI [35], OPAL [41] & $\bullet \bullet \bullet \bullet \bullet$ \\
\hline
\end{tabular}

Table 7: Overview of the combinations of identified particle data used for the HERWIG fits. Data used are marked by $\bullet$. The central fit result corresponds to the choice $P_{5}$. The other combinations were used to provide estimates of the systematic errors.

insets, are the total experimental errors obtained by adding quadratically the systematic and statistical error in each bin.

The different decay treatments lead to negligible differences for the stable charged particle and event shape distributions. Comparisons are made with the following models:

- JETSET 7.3 with DELPHI decays labeled JT 7.3 PS

- JETSET 7.4 default decays labeled JT 7.4 PS

- ARIADNE 4.06 with DELPHI decays labeled AR 4.06

- HERWIG 5.8 C default decays labeled H $5.8 \mathrm{C}$

- JETSET 7.4 ME default decays labeled JT 7.4 ME

The observations below are made from the model to data comparisons.

\subsection{Inclusive Charged Particle Spectra}

All models describe the general trends of the data well. Few discrepancies show up from direct model to data comparisons. More quantitatively, the comparisons of model and data (lower insets) show the following.

The $x_{p}$ spectrum (Fig. 4) for $x_{p}<0.4$ is almost perfectly described by the ARIADNE and JETSET PS models. At large $x_{p}$ these models slightly underestimate the data. This trend is reduced if the multiplicity is left free in the fit. The HERWIG and JETSET ME predictions alternate between being too high and too low with respect to the data. This behaviour is also reflected in the rapidity distributions (see Figs. 5, 6). 


\begin{tabular}{|c|c|c|c|c|c|}
\hline & \multicolumn{5}{|c|}{$\chi^{2} /$ bin } \\
\hline & ARIADNE & JETSET & JETSET & HERWIG & JETSET \\
\hline & 4.06 & $7.4 \mathrm{PS}$ & $7.3 \mathrm{PS}$ & $5.8 \mathrm{C}$ & $7.4 \mathrm{ME}$ \\
\hline $1-T$ & 0.67 & 0.98 & 1.14 & 8.44 & 27.23 \\
\hline$M$ & 1.41 & 1.99 & 2.34 & 21.50 & 11.83 \\
\hline$m$ & 1.28 & 3.43 & 4.10 & 38.17 & 18.89 \\
\hline$O$ & 0.58 & 7.78 & 7.62 & 1.33 & 6.24 \\
\hline$S$ & 1.07 & 2.29 & 2.28 & 4.32 & 3.52 \\
\hline$A$ & 1.57 & 5.57 & 5.53 & 7.56 & 13.32 \\
\hline$P$ & 0.91 & 2.25 & 1.97 & 2.29 & 6.17 \\
\hline$C$ & 1.00 & 1.86 & 2.09 & 12.48 & 25.77 \\
\hline$D$ & 1.55 & 3.36 & 3.58 & 8.84 & 29.62 \\
\hline$M_{\text {high }}^{2} / E_{\text {vis }}^{2}$ & 2.77 & 1.89 & 2.17 & 2.16 & 10.50 \\
\hline$M_{\text {low }}^{2} / E_{\text {vis }}^{2}$ & 0.65 & 0.77 & 0.60 & 1.40 & 4.90 \\
\hline$M_{d i f f}^{2} / E_{v i s}^{2}$ & 4.45 & 0.37 & 0.34 & 1.25 & 3.76 \\
\hline$B_{\max }$ & 2.31 & 1.67 & 2.42 & 25.78 & 10.18 \\
\hline$B_{\min }$ & 9.77 & 1.92 & 1.13 & 70.14 & 13.39 \\
\hline$B_{\text {sum }}$ & 1.50 & 2.19 & 2.70 & 16.65 & 10.22 \\
\hline$B_{\text {diff. }}$ & 5.40 & 3.11 & 3.22 & 0.99 & 1.21 \\
\hline$D_{2}^{D}$ & 1.12 & 2.37 & 3.33 & 1.57 & 2.16 \\
\hline$D_{3}^{D}$ & 1.23 & 3.28 & 2.60 & 2.15 & 1.12 \\
\hline$D_{4}^{D}$ & 2.13 & 3.87 & 4.44 & 7.15 & 65.05 \\
\hline$D_{2}^{J}$ & 0.56 & 2.15 & 2.54 & 5.02 & 5.79 \\
\hline$D_{3}^{J}$ & 0.63 & 6.97 & 5.75 & 1.92 & 5.72 \\
\hline$D_{4}^{J}$ & 2.70 & 7.53 & 8.13 & 10.34 & 82.91 \\
\hline$E E C$ & 0.27 & 1.48 & 1.62 & 0.93 & 5.39 \\
\hline$A E E C$ & 2.11 & 9.28 & 9.84 & 11.26 & 11.05 \\
\hline$p_{t}^{2 n} \quad$ T axis & 3.69 & 1.53 & 1.74 & 2.58 & 2.03 \\
\hline$p_{t}^{\text {out }} T$ axis & 17.27 & 26.48 & 26.68 & 11.79 & 28.84 \\
\hline$y_{T}$ & 1.11 & 1.07 & 1.43 & 9.09 & 40.95 \\
\hline$p_{t}^{i n} \quad S$ axis & 5.33 & 2.83 & 3.34 & 4.06 & 1.58 \\
\hline$p_{t}^{\text {out }} S$ axis & 14.01 & 20.78 & 21.74 & 7.51 & 21.39 \\
\hline$y_{S}$ & 1.38 & 0.93 & 1.44 & 1.57 & 11.63 \\
\hline$x_{p}$ & 2.22 & 0.98 & 1.76 & 3.76 & 3.55 \\
\hline$\xi_{p}$ & 1.30 & 0.99 & 0.95 & 3.41 & 2.48 \\
\hline$p_{t}^{o u t} \quad$ vs. $x_{p}$ & 14.39 & 23.68 & 19.04 & 25.76 & 56.20 \\
\hline$p_{t} \quad$ vs. $x_{p}$ & 11.00 & 4.48 & 5.32 & 19.12 & 25.41 \\
\hline all distrib. & 3.62 & 5.32 & 5.40 & 10.62 & 16.05 \\
\hline
\end{tabular}

Table 8: $\chi^{2} /$ bin for the model/data comparisons of event shape and inclusive charged particle distributions. Only the $p_{t}^{\text {out }}$ distributions are badly described by all models.

For all models, the $p_{t}^{\text {in }}$ distribution (Fig. 7) agrees with the data within errors. Only the large $p_{t}^{i n}$ tail is slightly underestimated.

Also for all models, the predicted $p_{t}^{\text {out }}$ distribution (Fig. 8) for $p_{t}^{\text {out }}>0.8 \mathrm{GeV}$ falls off more rapidly than the data and is about $30 \%$ below the data at large $p_{t}^{\text {out }}$. The large $p_{t}^{\text {out }}$ tail is due mainly to gluon radiation. For the ME model, a discrepancy might be expected because of missing higher order terms in the second order matrix element calculation. The failure of the parton shower models can possibly be traced back to missing large angle terms in the basic LLA formalism used by the models. If these reasons for the failure of the models are correct, a matching of the second order calculation and the LLA formalism should lead to an improved description of $p_{t}^{\text {out }}$ and related distributions.

\subsection{Event Shape Distributions}


The general event shape distributions, $1-T, S, C$ and $B_{\text {sum }}$ (Figs. 11, 21, 31 and 29 ), are well described within the small experimental errors (typically $2-3 \%$ ) by all the parton shower models. The HERWIG predictions tend to lie slightly above the data for large values of these observables, the JETSET ME predictions tend to oscillate around the data.

The description of distributions sensitive to transverse momenta in the event plane, like $M, B_{\max }$ or $M_{h}^{2} / E_{v i s}^{2}$ (Figs. 12, 27 or 25 ), is only slightly less good (typically better than $5 \%$ ).

Turning to distributions sensitive to transverse momenta out of the event plane, namely $m, O, A, M_{l}^{2} / E_{v i s}^{2}$, and $B_{\text {min }}$ (Figs. 13, 14, 22, 26, 28), the following pattern is observed: ARIADNE generally describes the data well, while for higher values of the observables, HERWIG tends to overestimate the distributions and JETSET PS to underestimate them. The latter is to be expected from the underestimation of the $p_{t}^{\text {out }}$ distribution by all PS models.

A similar pattern is also observed for the jet rates. The differential 2-jet rate $\mathrm{D}_{2}$ (Figs. 15, 16) is well described by all models. ARIADNE describes also the higher jet rates well (Figs. 17-20), HERWIG overestimates and JETSET PS underestimates them. This behaviour is similar for the JADE and DURHAM jet algorithms.

The event shape distributions are less well described by the JETSET ME model than by the PS models. The extreme 2-jet region, the multi-jet rates, and observables sensitive to radiation out of the event plane are also not described quite so well. Nevertheless, in general, the description by the JETSET ME model is reasonable.

To permit a comprehensive comparison of the different models, Table 8 shows the mean $\chi^{2} /$ bin obtained from the comparison of the individual data and model distributions. The $\chi^{2} /$ bin values shown should be used only for relative comparison, not to infer absolute confidence levels. In the limit of large statistics, even a slightly imperfect model description leads to a very large $\chi^{2} /$ bin. Furthermore, in principle, correlations between the different distributions should be taken into account.

\subsection{Identified Particle Rates}

Table 9 compares the particle rates predicted by the models with the current measured LEP averages. If the mean charged multiplicity $\left\langle N_{c h}\right\rangle$ is included in the fits, it is well described by the models. Neglecting this constraint, the multiplicity predicted by ARIADNE and JETSET PS is too low $(20.2-20.4)$ and that predicted by JETSET ME is too high (22.7). The HERWIG prediction is correct without constraint.

The meson rates, with the exception of the $K^{ \pm}$and the $\eta$ rates, are described fairly well. The $K^{ \pm}$rate is sensitive to heavy quark decays (see below). The octet baryons also agree reasonably. Only HERWIG overestimates the $\Xi^{-}$rate by about a factor 2 . But it gives the best prediction for the $\Omega^{-}$rate. There are some discrepancies in the decuplet baryon sector.

\subsection{Meson Momentum Spectra}

Figs. 39, 40 compare the models to the identified kaon spectra [13,33-37] as a function of $\xi_{p}=\log \frac{1}{x_{p}}$ from different LEP experiments. For a more quantitative comparison which also allows the agreement among the different experiments to be judged, the relative deviation between the individual data sets and each model is shown by the lines in the figures on the right. In the fragmentation region at large $\xi_{p}$ (i.e. small $x_{p}$ ), where the 


\begin{tabular}{|c|c|c|c|c|c|c|c|}
\hline \multirow{2}{*}{\multicolumn{2}{|c|}{$\begin{array}{r}\text { JETSET } \\
7.3 \text { PS } \\
\text { Charged Particles }\end{array}$}} & \multirow{2}{*}{$\begin{array}{l}\text { JETSET } \\
7.4 \mathrm{PS} \\
\end{array}$} & \multirow[t]{2}{*}{$\begin{array}{c}\text { ARIADNE } \\
4.06\end{array}$} & \multirow[t]{2}{*}{$\begin{array}{c}\text { JETSET } \\
7.4 \mathrm{ME}\end{array}$} & \multirow[t]{2}{*}{$\begin{array}{c}\text { HERWIG } \\
5.8 \mathrm{C}\end{array}$} & \multicolumn{2}{|c|}{$\begin{array}{c}\text { LEP } \\
{[38,43-45]}\end{array}$} \\
\hline & & & & & & & \\
\hline$<N_{c h}>$ & 20.87 & 20.81 & 20.80 & 20.86 & 20.94 & 20.92 & \pm 0.24 \\
\hline \multicolumn{8}{|c|}{ pseudoscalar mesons } \\
\hline$\pi^{ \pm}$ & 17.19 & 17.09 & 17.13 & 17.36 & 17.66 & 17.1 & \pm 0.4 \\
\hline$\pi^{0}$ & 9.85 & 9.83 & 9.82 & 10.03 & 9.81 & 9.9 & \pm 0.08 \\
\hline$K^{ \pm}$ & 2.20 & 2.23 & 2.19 & 2.15 & 2.11 & 2.42 & \pm 0.13 \\
\hline$K^{0}$ & 2.13 & 2.17 & 2.12 & 2.10 & 2.08 & 2.12 & \pm 0.06 \\
\hline$\eta$ & 1.07 & 1.10 & 1.09 & 1.16 & 1.02 & 0.73 & \pm 0.07 \\
\hline$\eta^{\prime}(958)$ & 0.10 & 0.09 & 0.10 & 0.10 & 0.14 & 0.17 & \pm 0.05 \\
\hline$D^{+}$ & 0.19 & 0.20 & 0.20 & 0.20 & 0.24 & 0.20 & \pm 0.03 \\
\hline$D^{0}$ & 0.46 & 0.49 & 0.48 & 0.49 & 0.53 & 0.40 & \pm 0.06 \\
\hline$B^{ \pm}, B^{0}$ & 0.36 & 0.36 & 0.36 & 0.36 & 0.36 & 0.34 & \pm 0.06 \\
\hline \multicolumn{8}{|c|}{ scalar mesons } \\
\hline$f_{0}(980)$ & 0.17 & 0.16 & 0.17 & 0.16 & & 0.14 & \pm 0.06 \\
\hline \multicolumn{8}{|c|}{ vector mesons } \\
\hline$\rho^{\circ}(770)$ & 1.29 & 1.27 & 1.26 & 1.29 & 1.43 & 1.40 & \pm 0.1 \\
\hline$K^{* \pm}(892)$ & 0.78 & 0.77 & 0.79 & 0.77 & 0.74 & 0.78 & \pm 0.08 \\
\hline$K^{* 0}(892)$ & 0.80 & 0.77 & 0.81 & 0.78 & 0.74 & 0.77 & \pm 0.09 \\
\hline$\phi(1020)$ & 0.109 & 0.107 & 0.107 & 0.102 & 0.099 & 0.086 & \pm 0.018 \\
\hline$D^{* \pm}(2010)$ & 0.18 & 0.22 & 0.19 & 0.22 & 0.22 & 0.17 & \pm 0.02 \\
\hline \multicolumn{8}{|c|}{ tensor mesons } \\
\hline$f_{2}(1270)$ & 0.29 & 0.29 & 0.29 & 0.30 & 0.26 & 0.31 & \pm 0.12 \\
\hline$K^{*}(1430)$ & 0.15 & 0.15 & 0.16 & 0.16 & 0.13 & 0.19 & \pm 0.07 \\
\hline \multicolumn{8}{|l|}{ baryons } \\
\hline$p$ & 0.97 & 0.97 & 0.96 & 0.90 & 0.78 & 0.92 & \pm 0.11 \\
\hline$\Lambda^{0}$ & 0.361 & 0.349 & 0.365 & 0.309 & 0.368 & 0.348 & \pm 0.013 \\
\hline$\Xi^{-}$ & 0.0288 & 0.0300 & 0.0300 & 0.0256 & 0.0493 & 0.0238 & \pm 0.0024 \\
\hline$\Omega^{-}$ & 0.0013 & 0.0019 & 0.0021 & 0.0010 & 0.0077 & 0.0051 & \pm 0.0013 \\
\hline$\Delta^{++}(1232)$ & 0.158 & 0.160 & 0.136 & 0.158 & 0.154 & 0.124 & \pm 0.065 \\
\hline$\Sigma^{ \pm}(1385)$ & 0.037 & 0.036 & 0.032 & 0.033 & 0.065 & 0.0380 & \pm 0.0062 \\
\hline$\Xi^{0}(1530)$ & 0.0073 & 0.0069 & 0.0063 & 0.0060 & 0.0249 & 0.0063 & \pm 0.0014 \\
\hline$\Lambda_{b}^{0}$ & 0.032 & 0.033 & 0.032 & 0.029 & 0.007 & 0.031 & \pm 0.016 \\
\hline
\end{tabular}

Table 9: Single particle production rates for the different generators compared to LEP I data.

$K^{ \pm}$data are more precise and therefore dominate the fit, the $K^{0}$ rates are overestimated by $\approx 10-20 \%$ by all models. At more central momenta, all kaons are well described. But in the range $1.0<\xi_{p}<2.5$, the $K^{ \pm}$rates are underestimated. This inconsistency between data and models causes the relatively large systematic error on $\gamma_{s}$.

Kaons from heavy particle decays tend to contribute mainly in this momentum range, so wrong descriptions of the decay of $b$-hadrons are a likely cause of this discrepancy. A recent DELPHI measurement [46] of the inclusive particle production in $b$-events found the difference between the $K^{ \pm}$and $K^{0}$ multiplicities to be $0.58 \pm 0.51$. The corresponding value in the models is $\approx 0.30$ for JETSET and ARIADNE and 0.04 for HERWIG. Improvement will be possible as soon as precise measurements of the momentum spectra of kaons for identified $b \bar{b}$ and light quark events become available.

Finally, at very small $\xi_{p}$ (i.e. large $x_{p}$ ) there is an indication that the models underestimate the $\mathrm{K}$ production slightly.

The fragmentation functions of vector mesons [35,39,41,42,47], which are likely to be primary particles, are compared to the data in Figs. 41, 42, 43. Within the large errors of these resonance measurements, all models describe the spectra very well (but note here that the parameterization of resonance production probabilities used here differs from the 
JETSET default, see section 3). There is only a tendency to predict a somewhat harder fragmentation than measured. This is most evident for the $\Phi(1020)$ spectrum.

Fig. 44 shows good agreement of the model predictions with the measurements of the $f_{0}(980)$ scalar and $f_{2}(1270)$ tensor meson [39]. With large production probabilities for p-wave resonances, all models describe the data well. Note that high production of p-wave states $(>10 \%)$ is not expected in the string picture [12].

\subsection{Baryon Momentum Spectra}

The proton spectra are compared in Fig. $45[33,34,36]$. There is a severe discrepancy between the OPAL and ALEPH results at small $\xi_{p}$. Since both data sets are used for the central fits, the fits interpolate between them.

For both data sets, the rate predicted by HERWIG is too high at small $\xi_{p}$ and too low at high $\xi_{p}$. At small $\xi_{p}$, i.e. high momentum, the behaviour of JETSET PS and ARIADNE is similar to that of HERWIG if the extra baryon suppression is not used. The need for an extra suppression of high momentum baryon production may indicate a different production mechanism for baryons than for mesons. It could also be partly due to orbitally excited states missing in the models [8]. However, these states have not yet been observed in $e^{+} e^{-}$annihilation.

The experimental situation for the $\Lambda^{0}$ spectrum $[13,35,48]$ also shows some discrepancies (see Fig. 46). JETSET PS and ARIADNE describe or slightly overestimate the small $\xi_{p}$ region. The HERWIG prediction is again too high. At large $\xi_{p}$, where the measurements agree, all models underestimate the $\Lambda^{0}$ production by $\approx 15 \%$. Better descriptions may be obtainable by restricting to the proton and $\Lambda^{0}$ spectra of specified experiments or by fitting all parameters relevant to baryon production simultaneously [50].

Fig. 47 shows in a comprehensive overview a comparison of the octet and decuplet baryon production $[13,35,48,49]$ with the model predictions. The discrepancies discussed above are hidden here, due to the large scales. JETSET and ARIADNE describe the gross features of the octet and decuplet baryon production well. HERWIG predicts too hard a baryon fragmentation, and also predicts the relative production rates of the different multiplet states less well.

\section{Summary}

Precise fully corrected inclusive charged particle and event shape distributions have been determined from $750000 e^{+} e^{-} \rightarrow Z \rightarrow$ hadrons events measured by the DELPHI experiment.

A systematic quantitative study has been undertaken to determine the optimal choice of distributions to tune fragmentation models. Semi-inclusive charged particle and identified particle distributions constrain the hadronization part of the models, whereas 3 -jet rate distributions and most event shape distributions mainly control the parton shower parameters (especially $\Lambda_{Q C D}$ ) in the models.

Optimum parameter values have been determined for the ARIADNE 4.06, HERWIG 5.8 $\mathrm{C}$ and JETSET 7.3 and 7.4 parton shower models and for the JETSET 7.4 matrix element model. The models were fitted to the event shape and inclusive charged particle distributions measured in this analysis and to the mean charged particle multiplicity and identified particle data measured by all the LEP experiments. The fit algorithm employed allowed a simultaneous fit of up to 10 model parameters. Statistical and systematic errors as well as correlations of the model parameters have been determined. 
All models describe the inclusive charged particle and event shape distributions reasonably well. The data measured from charged particles and charged plus neutral particles yield consistent results when compared with the corresponding model predictions. All models underestimate the tail of the $p_{t}^{\text {out }}$ distribution by more than $25 \%$. With this exception, the best overall description is provided by ARIADNE 4.06. HERWIG 5.8 C tends to overestimate and JETSET 7.3/7.4 to underestimate the production of 4 or more jet events. The tails of event shape distributions sensitive to particle production out of the event plane are overestimated (underestimated) by HERWIG (JETSET). The matrix element model JETSET 7.4 ME with optimized scale also provides reasonable predictions. However, it shows the expected discrepancies in the extreme 2-jet and multi-jet regions due to the missing higher order terms.

Identified meson spectra are fairly well described by all models. It has been found that strong production of $\mathrm{p}$-wave resonances $(25-40 \%)$ has to be considered. This is not expected in a string fragmentation picture. The gross features of baryon production are described by JETSET and ARIADNE. HERWIG shows stronger discrepancies, with the predicted fragmentation functions being too hard. In JETSET and ARIADNE, a similar tendency has been corrected by applying an extra leading baryon suppression.

\section{Acknowledgements}

We are greatly indebted to our technical collaborators and to the funding agencies for their support in building and operating the DELPHI detector, and to the CERN-SL Division for the superb performance of the LEP collider. We would like to thank G. Heindl, I. Knowles, L. Lönnblad, M. Seymour and T. Sjöstrand for useful discussions. 


\section{A Definition of Variables}

This paper uses the following definitions of event shape and inclusive particle variables:

\section{A.1 Inclusive Single Particle Variables}

Scaled Momentum, $x_{p}, \xi_{p}$

The scaled momentum, $x_{p}$, is the absolute momentum, $|\vec{p}|$, of a particle scaled to the beam momentum, while $\xi_{p}=\log \frac{1}{x_{p}}$.

Transverse Momenta, $p_{t}, p_{t}^{\text {in }}, p_{t}^{\text {out }}$

With respect to the thrust axis, the component of the transverse momentum $p_{t}$ of a particle in the event plane is $p_{t}^{i n}=\vec{p} \cdot \vec{n}_{\text {Major }}$ and that out of the event plane is $p_{t}^{\text {out }}=\vec{p} \cdot \vec{n}_{\text {Minor }}$. For the values with respect to the sphericity axis, the axes defined by the eigenvectors of the quadratic momentum tensor are used instead.

Rapidity $y$

The rapidity is given by:

$$
y=\frac{1}{2} \cdot \log \frac{E+p_{\|}}{E-p_{\|}}
$$

where $p_{\|}$is the particle momentum parallel to the event thrust axis $\vec{n}_{\text {Thrust }}$ for $y_{T}$, or the sphericity axis $\vec{n}_{\text {Sphericity }}$ for $y_{S}$.

\section{A.2 Event Shape Variables}

\section{Thrust $T$, Major $M$, Minor $m$, Oblateness $O$}

The Thrust [51], T, and the Thrust axis, $\vec{n}_{\text {Thrust }}$, are defined by:

$$
T=\max _{\vec{n}_{\text {Thrust }}} \frac{\sum_{i=1}^{N_{\text {particle }}}\left|\vec{p}_{i} \cdot \vec{n}_{\text {Thrust }}\right|}{\sum_{i=1}^{N_{\text {particle }}}\left|\vec{p}_{i}\right|}
$$

$\vec{n}_{\text {Thrust }}$ is a unit-vector along the Thrust axis. Major and Minor are defined similarly, replacing $\vec{n}_{\text {Thrust }}$ by $\vec{n}_{\text {Major }}$, perpendicular to $\vec{n}_{\text {Thrust }}$, and $\vec{n}_{\text {Minor }}=\vec{n}_{\text {Major }} \times \vec{n}_{\text {Thrust }}$ respectively. The Oblateness is $O=M-m$.

\section{Sphericity $S$, Aplanarity $A$, Planarity $P$}

Ordering the eigenvalues $\lambda$ of the quadratic momentum tensor:

$$
\begin{array}{rlr}
M^{\alpha \beta} & =\sum_{i=1}^{N_{\text {particle }}} p_{i}^{\alpha} p_{i}^{\beta} & (\alpha, \beta=1,2,3) \\
\lambda_{1} \geq \lambda_{2} \geq \lambda_{3} & \lambda_{1}+\lambda_{2}+\lambda_{3}=1
\end{array}
$$

The Sphericity is $S=\frac{3}{2}\left(\lambda_{2}+\lambda_{3}\right)$, the Aplanarity is $A=\frac{3}{2} \lambda_{3}$, and the Planarity is $P=\frac{2}{3}(S-2 A)$ [52]. The Sphericity axis is parallel to the eigenvector corresponding to $\lambda_{1}$. As the momenta enter quadratically, the Sphericity axis is influenced more strongly by large momentum particles than the Thrust axis. 
The $\mathrm{C}$ and $\mathrm{D}$ Parameters are defined through the eigenvalues $\lambda$ of the linear momentum tensor [53]:

$$
\begin{gathered}
\Theta^{i, j}=\frac{1}{\sum_{k=1}^{N_{\text {particle }}}\left|\vec{p}_{k}\right|} \cdot \sum_{k=1}^{N_{\text {particle }}} \frac{p_{k}^{i} p_{k}^{j}}{\left|\vec{p}_{k}\right|} \\
C=3 \cdot\left(\lambda_{1} \lambda_{2}+\lambda_{2} \lambda_{3}+\lambda_{3} \lambda_{1}\right) \quad D=27 \cdot \lambda_{1} \lambda_{2} \lambda_{3}
\end{gathered}
$$

Hemisphere Masses $M_{\text {high }}^{2} / E_{v i s}^{2}, M_{\text {low }}^{2} / E_{v i s}^{2}, M_{\text {diff }}^{2} / E_{v i s}^{2}$ (also called Jet Masses)

Particles are ordered in the two hemispheres of an event separated by the plane normal to $\vec{n}_{\text {Thrust }}$. Then

$$
\frac{M_{\text {high }}^{2}}{E_{v i s}^{2}}=\frac{1}{E_{v i s}^{2}} \cdot \max \left(\left(\sum_{\vec{p}_{k} \cdot \vec{n}_{T h r u s t}>0} p_{k}\right)^{2},\left(\sum_{\vec{p}_{k} \cdot \vec{n}_{T h r u s t}<0} p_{k}\right)^{2}\right)
$$

For $\frac{M_{l o w}^{2}}{E_{v i s}^{2}}$ the maximum in the above formula is replaced by the minimum, and $\frac{M_{\text {diff }}^{2}}{E_{v i s}^{2}}=\frac{M_{h i g h}^{2}}{E_{v i s}^{2}}-\frac{M_{\text {low }}^{2}}{E_{v i s}^{2}}$. This definition differs from the original one by Clavelli [54], but is easier to calculate and therefore preferred by many experiments.

Hemisphere Broadening $B_{\max }, B_{\min }, B_{\text {sum }}, B_{\text {diff }}$ (also called Jet Broadening) In each hemisphere, defined in the same way as for the hemisphere masses, the momenta transverse to the thrust axis are summed and divided by the corresponding sum of the absolute momenta [55], i.e.

$$
\begin{gathered}
B_{ \pm}=\frac{\sum_{ \pm \vec{p}_{i} \cdot \vec{n}_{\text {Thrust }}>0}\left|\vec{p}_{i} \times \vec{n}_{\text {Thrust }}\right|}{2 \sum_{i}\left|\vec{p}_{i}\right|} \\
B_{\text {max }}=\max \left(B_{+}, B_{-}\right) ; \quad B_{\text {min }}=\min \left(B_{+}, B_{-}\right) ; \\
B_{\text {sum }}=B_{+}+B_{-} ; \quad B_{\text {diff }}=\left|B_{+}-B_{-}\right| .
\end{gathered}
$$

Differential Jet Rates $D_{i}(y)$

Jets are reconstructed using cluster finding algorithms. For the JADE [56] or Durham [57] algorithm respectively, the scaled invariant mass or transverse momentum $y_{i j}$ :

$$
\begin{gathered}
y_{i j}^{\text {Jade }}=2 E_{i} E_{j} / E_{v i s}^{2} \cdot\left(1-\cos \theta_{i j}\right) \\
y_{i j}^{\text {Durham }}=2 \min \left(E_{i}^{2}, E_{j}^{2}\right) / E_{v i s}^{2} \cdot\left(1-\cos \theta_{i j}\right)
\end{gathered}
$$

is evaluated for each pair of particles $i$ and $j$ in an event, where $E_{i}, E_{j}$ are the energies and $\theta_{i j}$ the angle between the momentum vectors of the two particles. The particle pair with the lowest value of $y_{i j}$ is replaced by a pseudo-particle with 4 -momentum $\left(p_{i}+p_{j}\right)$, reducing the multiplicity by one. The procedure is repeated until the scaled invariant masses of all pairs of (pseudo-)particles exceed a given resolution $y$. The remaining (pseudo-)particles are called 
jets. The differential 2-jet rate $D_{2}$ is derived from the 2-jet rate $R_{2}=N_{2-j e t s} / N[58]$ :

$$
D_{2}(y)=\frac{R_{2}(y+\Delta y)-R_{2}(y)}{\Delta y}
$$

Higher differential jet rates follow from the recursion:

$$
D_{n}(y)=\frac{R_{n}(y+\Delta y)-R_{n}(y)}{\Delta y}+D_{n-1}(y)
$$

\section{Energy-Energy-Correlation $E E C$ and Asymmetry $A E E C$}

The Energy-Energy-Correlation EEC is the histogram of angles, $\chi_{i j}$, between all particles weighted by their scaled energies [59]:

$E E C(\cos \chi)=\frac{1}{N} \frac{1}{\Delta \cos \chi} \cdot \sum_{\text {events }}^{N} \sum_{i<j} \frac{E_{i}}{E_{v i s}} \frac{E_{j}}{E_{v i s}} \Theta\left(\Delta \cos \chi-\left|\cos \chi-\cos \chi_{i j}\right|\right)$

where $\cos \chi$ and $\Delta \cos \chi$ are the lower edge and width of a bin respectively, and $\Theta$ is the step function. For $\cos \chi \geq 0$, the asymmetry $A E E C$ of the $E E C$ is:

$$
A E E C(\cos \chi)=E E C(-\cos \chi)-E E C(\cos \chi)
$$




\section{B Parameter Settings of the DELPHI Monte Carlo}

The DELPHI tuning of JETSET has been obtained by tuning the model to charged particle data from the 1991 and 1992 data taking. Care has been taken to describe the observables relevant to standard precision analyses which are the charged multiplicity, the momentum spectrum, 2-jet rate and Thrust and Sphericity distribution. The simulation also includes Bose Einstein interference (by LUBOEI) to obtain a correct description of two particle correlations and light resonance line shapes. The BE parameters are taken from the DELPHI measurement [60]. Particle spectra have been adjusted and partially fitted to available data [45]. Heavy particle decays have been adjusted.

\begin{tabular}{|c|c|c|}
\hline Variable & Dec. 93 & Sept. 94 \\
\hline MSTJ(11) & 3 & 3 \\
\hline MSTJ(12) & 3 & 3 \\
\hline $\operatorname{MSTJ(41)}$ & 2 & 2 \\
\hline $\operatorname{MSTJ}(45)$ & 5 & 5 \\
\hline $\operatorname{MSTJ}(46)$ & 3 & 3 \\
\hline $\operatorname{MSTJ}(51)$ & 2 & 2 \\
\hline MSTJ(52) & 7 & 7 \\
\hline MSTJ(101) & 5 & 5 \\
\hline MSTJ(107) & 0 & 0 \\
\hline PARJ(1) & 0.10 & 0.10 \\
\hline PARJ(2) & 0.28 & 0.28 \\
\hline PARJ(3) & 0.55 & 0.55 \\
\hline PARJ(4) & 0.07 & 0.07 \\
\hline PARJ(5) & 0.5 & 0.5 \\
\hline PARJ(11) & 0.55 & \\
\hline PARJ(12) & 0.55 & \\
\hline PARJ(13) & 0.75 & \\
\hline PARJ(14) & 0.090 & \\
\hline PARJ(15) & 0.070 & \\
\hline PARJ(16) & 0.085 & \\
\hline PARJ(17) & 0.140 & \\
\hline PARJ(19) & 0.5 & 0.5 \\
\hline PARJ(21) & 0.417 & 0.428 \\
\hline PARJ(25) & 0.7 & 0.7 \\
\hline PARJ(26) & 0.2 & 0.2 \\
\hline PARJ(41) & 0.5 & 0.354 \\
\hline PARJ(42) & 0.701 & 0.523 \\
\hline PARJ(54) & -0.0631 & -0.0305 \\
\hline PARJ(55) & -0.00414 & -0.00233 \\
\hline
\end{tabular}

\begin{tabular}{lcc}
\hline Variable & Dec. 93 & Sept. 94 \\
\hline PARJ $(81)$ & 0.297 & 0.346 \\
PARJ $(82)$ & 1.732 & 2.25 \\
PARJ $(92)$ & 1 & 1 \\
PARJ $(93)$ & 0.394 & 0.394 \\
$P\left({ }^{1} S_{0}\right)_{u d}$ & & .423 \\
$P\left({ }^{3} S_{1}\right)_{u d}$ & & .275 \\
$P\left({ }^{1} P_{1}\right)_{u d}$ & & .067 \\
$P\left({ }^{3} P_{0}\right)_{u d}$ & & .056 \\
$P\left({ }^{3} P_{1}\right)_{u d}$ & & .067 \\
$P\left({ }^{3} P_{2}\right)_{u d}$ & & .112 \\
$P\left({ }^{1} S_{0}\right)_{s}$ & & .388 \\
$P\left({ }^{3} S_{1}\right)_{s}$ & & .296 \\
$P\left({ }^{1} P_{1}\right)_{s}$ & & .079 \\
$P\left({ }^{3} P_{0}\right)_{s}$ & & .026 \\
$P\left({ }^{3} P_{1}\right)_{s}$ & & .079 \\
$P\left({ }^{3} P_{2}\right)_{s}$ & & .132 \\
$P\left({ }^{1} S_{0}\right)_{c}$ & & .250 \\
$P\left({ }^{3} S_{1}\right)_{c}$ & & .400 \\
$P\left({ }^{1} P_{1}\right)_{c}$ & & .087 \\
$P\left({ }^{3} P_{0}\right)_{c}$ & .030 \\
$P\left({ }^{3} P_{1}\right)_{c}$ & .087 \\
$P\left({ }^{3} P_{2}\right)_{c}$ & .146 \\
$P\left({ }^{1} S_{0}\right)_{b}$ & .1625 \\
$P\left({ }^{3} S_{1}\right)_{b}$ & .4875 \\
$P\left({ }^{1} P_{1}\right)_{b}$ & & .087 \\
$P\left({ }^{3} P_{0}\right)_{b}$ & .030 \\
$P\left({ }^{3} P_{1}\right)_{b}$ & .087 \\
$P\left({ }^{3} P_{2}\right)_{b}$ & & .146 \\
\hline & &
\end{tabular}

Table 10: DELPHI parameter settings of JETSET 7.3 PS for the December 1993 tuning used for modelling detector effects for 1993 data and for the September 1994 tuning used for modelling detector effects for 1994 data. For 1994 the meson spin parameters, PARJ(11) to PARJ(17), have been replaced by the more detailed parameters, $P\left({ }^{2 s+1} L_{j}\right)_{q}$, where $q$ is the heavier quark type. 


\section{Tables of Inclusive Charged Particle and Event Shape Distributions}

The normalized differential distributions were determined from charged particles only and from charged plus neutral particles. The tables show first the measurement from charged particles corrected to the charged hadronic final state and then the measurement from charged plus neutral particles corrected to the full hadronic final state. For the inclusive charged particle distributions, only the relevant event axes have been evaluated for the corresponding final states. The statistical and systematic errors are also given.

\section{C.1 Tables of Inclusive Charged Particle Distributions}

\begin{tabular}{|c|c|c|c|c|}
\hline \multicolumn{4}{|c|}{$p_{t}^{2 n}$ with respect to the Thrust axis } & charged particles $\frac{1}{N} \frac{d n}{d p_{t}^{2 n}}\left[\mathrm{GeV}^{-1}\right]$ \\
\hline Interval [GeV] & \multicolumn{3}{|c|}{$\begin{array}{l}\text { axis definition from } \\
\text { seen charged particles } \\
\text { corrected to final } \\
\text { charged particles }\end{array}$} & $\begin{array}{c}\text { axis definition from } \\
\text { seen charged plus neutral particles } \\
\text { corrected to final } \\
\text { charged plus neutral particles }\end{array}$ \\
\hline $0.000-0$ & 367 & \pm 0.036 & \pm 1.653 & \pm 0.037 \\
\hline $0.100-0.200$ & & \pm 0.033 & \pm 1 & \pm 1.092 \\
\hline $0.200-0.300$ & 29.429 & \pm 0.028 & \pm 0.603 & \pm 0.028 \\
\hline $0.300-0.400$ & 21.326 & \pm 0.024 & \pm 0 & \pm 0.024 \\
\hline $0.400-0.500$ & 15.547 & \pm 0.020 & \pm 0 & \pm 0.219 \\
\hline $0.500-0.600$ & 11.463 & \pm 0.017 & \pm 0 & \pm 0.150 \\
\hline $0.600-0.700$ & 68 & \pm 0.015 & \pm 0 & \pm 0.111 \\
\hline $0.700-0.800$ & 6.664 & \pm 0.013 & \pm 0 & \pm 0.013 \\
\hline $0.800-1.000$ & 4.6916 & \pm 0.0078 & \pm 0.0 & $4.5131 \pm 0.0076 \pm 0.0624$ \\
\hline $1.000-1.200$ & 3.0694 & \pm 0.0063 & \pm 0 & $2.9522 \pm 0.0062 \pm 0.0420$ \\
\hline $1.200-1.400$ & 2.0913 & \pm 0.0052 & \pm 0.0298 & $2.0401 \pm 0.0052 \pm 0.0299$ \\
\hline $1.400-1.600$ & 1.5030 & \pm 0.0044 & \pm( & $1.4597 \pm 0.0044 \pm 0.0222$ \\
\hline $1.600-1.800$ & 1.0916 & \pm 0.0038 & \pm 0.0163 & $1.0796 \pm 0.0038 \pm 0.0171$ \\
\hline $1.800-2.000$ & 0.8306 & \pm 0.0033 & \pm 0.0130 & $0.8155 \pm 0.0033 \pm 0.0136$ \\
\hline $2.000-2.500$ & 0.5311 & \pm 0.0017 & \pm 0.0089 & $0.5326 \pm 0.0017 \pm 0.0095$ \\
\hline $2.500-3.000$ & 0.2946 & \pm 0.0013 & \pm 0.0055 & $0.2988 \pm 0.0013 \pm 0.0057$ \\
\hline $3.000-3.500$ & 0.174 & \pm 0.00097 & \pm 0.00372 & $0.18067 \pm 0.00099 \pm 0.0038$ \\
\hline $3.500-4.000$ & 0.10909 & \pm 0.00077 & \pm 0.00276 & $0.11471 \pm 0.00079 \pm 0.00273$ \\
\hline $4.000-5.000$ & 0.05911 & \pm 0.00041 & \pm 0.00182 & $0.06305 \pm 0.00042 \pm 0.0017$ \\
\hline $5.000-6.000$ & 0.02669 & \pm 0.00028 & \pm 0.00101 & $0.03040 \pm 0.00029 \pm 0.00095$ \\
\hline $6.000-7.000$ & 0.01369 & \pm 0.00021 & \pm 0.00064 & $0.01501 \pm 0.00021 \pm 0.00054$ \\
\hline $7.000-8.000$ & 0.0067 & \pm 0.00014 & \pm 0.00039 & $0.00858 \pm 0.00016 \pm 0.00035$ \\
\hline $8.000-10.000$ & 0.0027 & \pm 0.00006 & \pm 0.00019 & $0.00376 \pm 0.00008 \pm 0.00017$ \\
\hline $10.000-12.000$ & 0.00095 & \pm 0.00004 & \pm 0.00008 & $0.00123 \pm 0.00004 \pm 0.00006$ \\
\hline $12.000-14.000$ & 0.00031 & \pm 0.00002 & \pm 0.00003 & $0.00044 \pm 0.00003 \pm 0.00002$ \\
\hline
\end{tabular}

Table 11: Transverse momentum, $p_{t}^{\text {in }}$, with respect to the Thrust axis 


\begin{tabular}{|c|c|c|c|c|c|c|c|}
\hline \multicolumn{5}{|c|}{$p_{t}^{\text {out }}$ with respect to the Thrust axis } & \multicolumn{3}{|c|}{ charged particles $\frac{1}{N} \frac{d n}{d p_{t}^{\text {out }}}\left[\mathrm{GeV}^{-1}\right]$} \\
\hline \multicolumn{2}{|c|}{ Interval [GeV] } & \multicolumn{3}{|c|}{$\begin{array}{l}\text { axis definition from } \\
\text { seen charged particles } \\
\text { corrected to final } \\
\text { charged particles } \\
\end{array}$} & \multicolumn{3}{|c|}{$\begin{array}{c}\text { axis definition from } \\
\text { seen charged plus neutral particles } \\
\text { corrected to final } \\
\text { charged plus neutral particles }\end{array}$} \\
\hline $0.000-$ & 0.100 & .512 & \pm 0.043 & \pm 1.956 & 66.160 & \pm 0.043 & \pm 1.822 \\
\hline $00-$ & & 17 & \pm 0 & \pm 1 & & 37 & \pm \\
\hline $00-$ & 0.300 & 3.618 & \pm 0 & \pm 0 & & 030 & \pm 0.678 \\
\hline $00-$ & & 28 & \pm 0 & \pm & & 024 & 97 \\
\hline $0.400-$ & 0.500 & 13.160 & \pm 0.019 & \pm 0 & 13. & \pm 0.019 & \pm 0.239 \\
\hline $0.500-$ & 0.600 & 83 & \pm 0 & \pm 0 & & .015 & \pm \\
\hline $0.600-$ & 0.700 & 5.178 & \pm 0.012 & \pm 0 & 5.2 & \pm 0.012 & \pm 0.097 \\
\hline $0.700-$ & 0.800 & 455 & \pm 0.0095 & \pm 0.0 & 3. & \pm 0.0098 & \pm 0.0658 \\
\hline $0.800-$ & 1.000 & 1.8640 & \pm 0.0050 & \pm 0 & 09 & \pm 0.0052 & \pm 0.0398 \\
\hline $1.000-$ & 1.200 & 0.8873 & \pm 0.0035 & \pm 0.0210 & 0.9959 & \pm 0.0037 & \pm 0.0216 \\
\hline $1.200-$ & 1.400 & 0.4600 & \pm 0.0026 & \pm 0.0 & 0.5288 & \pm 0.002 & \pm 0.0127 \\
\hline $1.400-$ & 1.600 & 0.2500 & \pm 0.0020 & \pm 0.0068 & 0.2987 & \pm 0.0021 & \pm 0.0079 \\
\hline $1.600-$ & 1.800 & 0.1429 & \pm 0.0015 & \pm 0.0042 & 0.1755 & \pm 0.001 & \pm 0.0051 \\
\hline $1.800-$ & 2.000 & 0.0841 & \pm 0.0011 & \pm 0.0026 & 0.1086 & \pm 0.0013 & \pm 0.0034 \\
\hline $2.000-$ & 2.500 & 0.04069 & \pm 0.00052 & \pm 0.00140 & 0.0526 & \pm 0.000 & \pm 0.00189 \\
\hline $2.500-$ & 3.000 & 0.01468 & \pm 0.00033 & \pm 0.00057 & 0.0188 & 土 0.000 & \pm 0.00080 \\
\hline $3.000-$ & 3.500 & 0.00501 & \pm 0.00018 & \pm 0.00022 & 0.0081 & \pm 0.0002 & \pm 0.00040 \\
\hline
\end{tabular}

Table 12: Transverse momentum, $p_{t}^{\text {out }}$, with respect to the Thrust axis

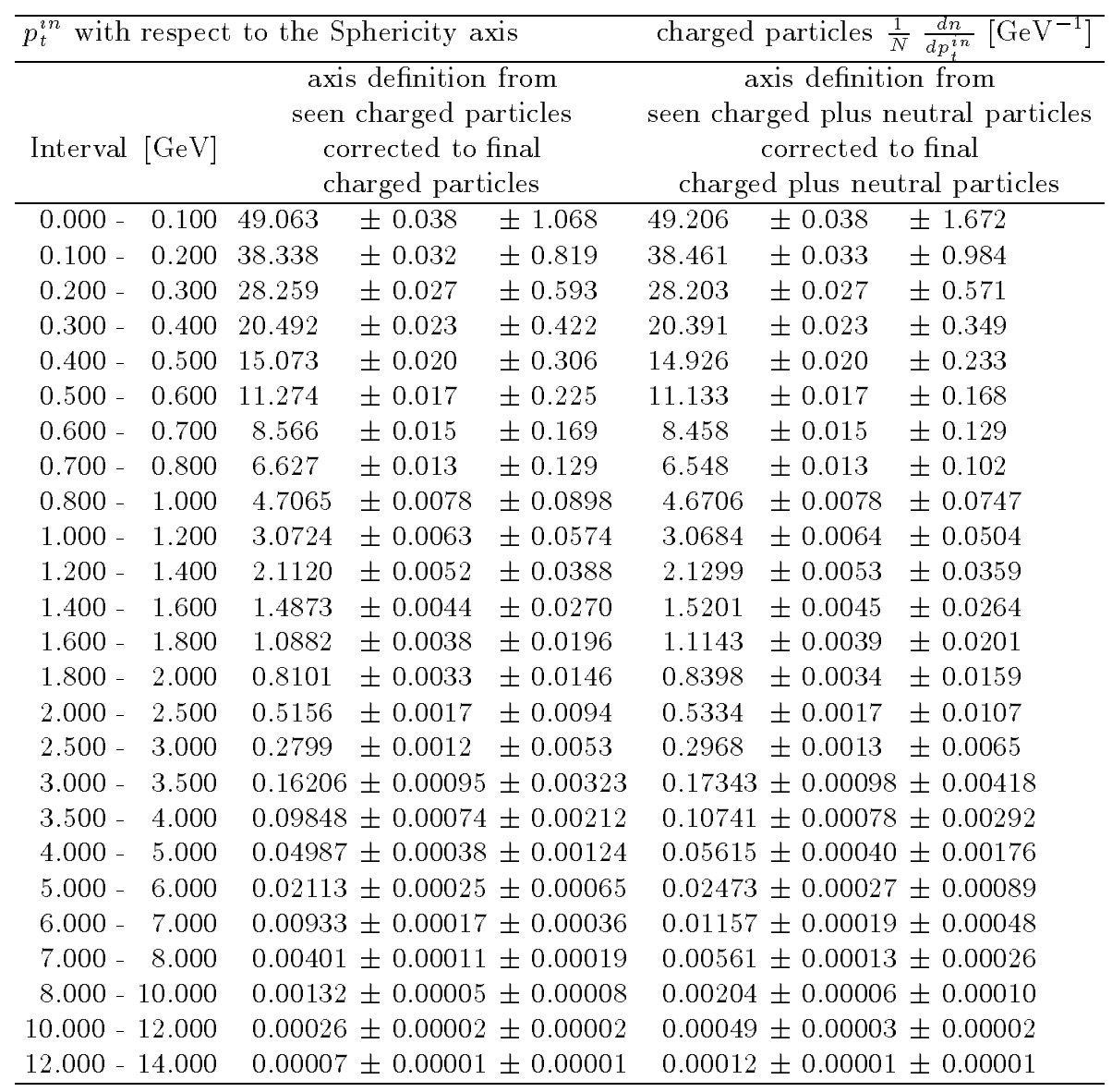

Table 13: Transverse momentum, $p_{t}^{\text {in }}$, with respect to the Sphericity axis 


\begin{tabular}{|c|c|c|c|c|c|}
\hline \multicolumn{5}{|c|}{$p_{t}^{\text {out }}$ with respect to the Sphericity axis } & charged particles $\frac{1}{N} \frac{d n}{d p_{t}^{o u t}}\left[\mathrm{GeV}^{-1}\right]$ \\
\hline \multicolumn{2}{|c|}{ Interval $[\mathrm{GeV}]$} & \multicolumn{3}{|c|}{$\begin{array}{l}\text { axis definition from } \\
\text { seen charged particles } \\
\text { corrected to final } \\
\text { charged particles }\end{array}$} & $\begin{array}{c}\text { axis definition from } \\
\text { seen charged plus neutral particles } \\
\text { corrected to final } \\
\text { charged plus neutral particles }\end{array}$ \\
\hline $0.000-$ & 0 & 848 & \pm 0.044 & \pm 1.931 & \pm 0.043 \\
\hline $00-$ & 0.200 & & \pm 0 & \pm 1 & \pm 1.102 \\
\hline $00-$ & 0.300 & 34 & \pm 0.030 & \pm 0 & \pm 0.726 \\
\hline $00-$ & 0.400 & 21.343 & \pm 0.024 & \pm 0 & \pm 0.024 \\
\hline $00-$ & 0.500 & 12 . & \pm 0.018 & \pm 0 & \pm 0.277 \\
\hline $00-$ & 0.600 & 7.765 & \pm 0.014 & \pm 0 & \pm 0.015 \\
\hline $00-$ & 0.700 & 57 & \pm 0.011 & \pm 0 & \pm 0.012 \\
\hline $00-$ & 0.800 & 543 & \pm 0.0090 & \pm 0 & $986 \pm 0.0094 \pm 0.0679$ \\
\hline $0.800-$ & 1.000 & 5438 & \pm 0.0047 & \pm 0.0373 & $1.7559 \pm 0.0049 \pm 0.0370$ \\
\hline $1.000-$ & 1.200 & 0.6885 & \pm 0.0032 & \pm 0.0186 & $0.8187 \pm 0.0034 \pm 0.0181$ \\
\hline $1.200-$ & 1.400 & 0.3309 & \pm 0.0023 & \pm 0.0099 & $0.4064 \pm 0.0024 \pm 0.0096$ \\
\hline $1.400-$ & 1.600 & 0.1730 & \pm 0.0017 & \pm 0.0057 & $0.2175 \pm 0.0018 \pm 0.0055$ \\
\hline $1.600-$ & 1.800 & 0.0956 & \pm 0.0013 & \pm 0.0034 & $0.1232 \pm 0.0014 \pm 0.0034$ \\
\hline $1.800-$ & 2.000 & 0.0518 & \pm 0.0009 & \pm 0.00199 & $0.0712 \pm 0.0011 \pm 0.0022$ \\
\hline $2.000-$ & 2.500 & 0.02330 & \pm 0.0004 & \pm 0.00101 & $0.03217 \pm 0.00047 \pm 0.00115$ \\
\hline $2.500-$ & 3.000 & 0.0072 ? & \pm 0.0002 & \pm 0.00037 & $0.01112 \pm 0.00029 \pm 0.00050$ \\
\hline $3.000-$ & 3.500 & 0.0023 & \pm 0.0001 & \pm 0.00013 & $0.00387 \pm 0.00017 \pm 0.00021$ \\
\hline
\end{tabular}

Table 14: Transverse momentum, $p_{t}^{\text {out }}$, with respect to the Sphericity axis

\begin{tabular}{|c|c|c|}
\hline \multicolumn{2}{|c|}{ Rapidity $y_{T}$ with respect to the Thrust axis } & charged particles $\frac{1}{N} \frac{d n}{d y_{T}}$ \\
\hline Interval & $\begin{array}{l}\text { axis definition from } \\
\text { seen charged particles } \\
\text { corrected to final } \\
\text { charged particles }\end{array}$ & $\begin{array}{c}\text { axis definition from } \\
\text { seen charged plus neutral particles } \\
\text { corrected to final } \\
\text { charged plus neutral particles }\end{array}$ \\
\hline $0.000-0.250$ & $5.7889 \pm 0.0093 \quad \pm 0.5509$ & $5.9517 \pm 0.0095 \quad \pm 0.5628$ \\
\hline 0.250 & $6.3749 \pm 0.0094 \pm 0$. & $6.4291 \pm$ \\
\hline $0.500-0.750$ & $6.6630 \pm 0.0093 \pm 0.3197$ & $6.6831 \pm 0.0094 \pm 0.3319$ \\
\hline $0.750-$ & $6.7572 \pm 0.0$ & $7763 \pm$ \\
\hline $1.000-1.250$ & $6.7638 \pm 0.0084 \pm 0.1677$ & $6.7650 \pm 0.0085 \pm 0.1755$ \\
\hline $1.250-1.500$ & $6.7463 \pm 0.0082 \pm 0$. & $6.7230 \pm 0.0083 \pm$ \\
\hline $1.500-1.750$ & $6.6488 \pm 0.0081 \pm 0.0933$ & $6.6085 \pm 0.0081 \pm 0.0939$ \\
\hline-2.000 & $6.5006 \pm 0.0080 \pm 0.0729$ & $6.4346 \pm 0.0080 \pm 0.0710$ \\
\hline $2.000-2.250$ & $6.2303 \pm 0.0079 \pm 0$ & $.1697 \pm 0.0078 \pm 0.0617$ \\
\hline $2.250-2.500$ & $5.8228 \pm 0.0076 \pm 0.0582$ & $5.7692 \pm 0.0076 \pm 0.0577$ \\
\hline $2.500-2.750$ & $5.1930 \pm 0.0072 \pm 0.0519$ & $5.1450 \pm 0.0072 \pm 0.0514$ \\
\hline $2.750-3.000$ & $4.3686 \pm 0.0067 \pm 0.0437$ & $4.3511 \pm 0.0066 \pm 0.0435$ \\
\hline $3.000-3.250$ & $3.4544 \pm 0.0059 \pm 0.0345$ & $3.4481 \pm 0.0059 \pm 0.0345$ \\
\hline $3.250-3.500$ & $2.5583 \pm 0.0051 \pm 0.0256$ & $2.5852 \pm 0.0051 \pm 0.0259$ \\
\hline $3.500-3.750$ & $1.7687 \pm 0.0042 \pm 0.0177$ & $1.7999 \pm 0.0043 \pm 0.0180$ \\
\hline $3.750-4.000$ & $1.1330 \pm 0.0034 \pm 0.0113$ & $1.1669 \pm 0.0035 \pm 0.0117$ \\
\hline $4.000-4.250$ & $0.6770 \pm 0.0026 \pm 0.0068$ & $0.7054 \pm 0.0027 \pm 0.0071$ \\
\hline $4.250-4.500$ & $0.3814 \pm 0.0019 \pm 0.0038$ & $0.3997 \pm 0.0020 \pm 0.0041$ \\
\hline $4.500-5.000$ & $0.14699 \pm 0.00084 \pm 0.00147$ & $0.15673 \pm 0.00089 \pm 0.00177$ \\
\hline $5.000-5.500$ & $0.03148 \pm 0.00038 \pm 0.00031$ & $0.03374 \pm 0.00041 \pm 0.00043$ \\
\hline $5.500-6.000$ & $0.00486 \pm 0.00016 \pm 0.00005$ & $0.00502 \pm 0.00016 \pm 0.00007$ \\
\hline
\end{tabular}

Table 15: Rapidity, $y_{T}$, with respect to the Thrust axis 


\begin{tabular}{|c|c|c|c|c|c|c|}
\hline \multicolumn{4}{|c|}{ Rapidity $y_{S}$ with respect to the Sphericity axis } & \multicolumn{3}{|c|}{ charged particles $\frac{1}{N} \frac{d n}{d y_{S}}$} \\
\hline Interval & \multicolumn{3}{|c|}{$\begin{array}{l}\text { axis definition from } \\
\text { seen charged particles } \\
\text { corrected to final } \\
\text { charged particles } \\
\end{array}$} & \multicolumn{3}{|c|}{$\begin{array}{c}\text { axis definition from } \\
\text { seen charged plus neutral particles } \\
\text { corrected to final } \\
\text { charged plus neutral particles }\end{array}$} \\
\hline $0.000-0.250$ & 6.5422 & \pm 0.0096 & \pm 0.5337 & 6.5680 & \pm 0.0097 & \pm 0.5323 \\
\hline $0.250-0.500$ & 6.5743 & \pm 0.0096 & \pm 0.4132 & 6.5901 & \pm 0.0096 & \pm 0.4246 \\
\hline $0.500-0.750$ & 6.5997 & \pm 0.0092 & \pm 0.3151 & 6.6094 & \pm 0.0093 & \pm 0.3329 \\
\hline $0.750-1.000$ & 6.5896 & \pm 0.0087 & \pm 0.2357 & 6.6152 & \pm 0.0088 & \pm 0.2554 \\
\hline $1.000-1.250$ & 6.5660 & \pm 0.0083 & \pm 0.1734 & 6.5917 & \pm 0.0084 & \pm 0.1908 \\
\hline $1.250-1.500$ & 6.5623 & \pm 0.0081 & \pm 0 & 6.5817 & \pm 0. & 393 \\
\hline $1.500-1.750$ & 6.4983 & \pm 0.0080 & \pm 0.0911 & 6.5221 & \pm 0.0081 & \pm 0.0983 \\
\hline $1.750-2.000$ & 6.3665 & \pm 0.0080 & \pm 0.0656 & 6.4097 & \pm 0.0080 & \pm 0.0673 \\
\hline $2.000-2.250$ & 6.1189 & \pm 0.0078 & \pm 0.0612 & 6.1741 & \pm 0.0079 & \pm 0.0617 \\
\hline $2.250-2.500$ & 5.6636 & \pm 0.0076 & \pm 0.0566 & 5.7542 & \pm 0.0076 & \pm 0.0575 \\
\hline $2.500-2.750$ & 5.0282 & \pm 0.0071 & \pm 0.0503 & 5.1066 & \pm 0.0072 & 511 \\
\hline $2.750-3.000$ & 4.2102 & \pm 0.0066 & \pm 0.0421 & 4.2721 & \pm 0.0066 & \pm 0.0427 \\
\hline $3.000-3.250$ & 3.3399 & \pm 0.0058 & \pm 0.0334 & 3.3718 & \pm 0.0059 & \pm 0.0337 \\
\hline $3.250-3.500$ & 2.5260 & \pm 0.0051 & \pm 0.0253 & 2.5185 & \pm 0.0051 & \pm 0.0252 \\
\hline $3.500-3.750$ & 1.7861 & \pm 0.0042 & \pm 0.0179 & 1.7588 & \pm 0.0042 & \pm 0.0176 \\
\hline $3.750-4.000$ & 1.2194 & \pm 0.0035 & \pm 0.0142 & 1.1589 & \pm 0.0034 & \pm 0.0130 \\
\hline $4.000-4.250$ & 0.8020 & \pm 0.0028 & \pm 0.0110 & 0.7327 & \pm 0.0027 & \pm 0.0105 \\
\hline $4.250-4.500$ & 0.4995 & \pm 0.0022 & \pm 0.0080 & 0.4402 & \pm 0.0021 & \pm 0.0078 \\
\hline $4.500-5.000$ & 0.2475 & \pm 0.0011 & \pm 0.0049 & 0.1952 & \pm 0.0010 & \pm 0.0046 \\
\hline $5.000-5.500$ & 0.08078 & \pm 0.00060 & \pm 0.00203 & 0.05574 & \pm 0.00050 & \pm 0.00180 \\
\hline $5.500-6.000$ & 0.0238 & \pm 0.00032 & \pm 0.00073 & 0.01306 & \pm 0.0002 & \pm 0.00055 \\
\hline
\end{tabular}

Table 16: Rapidity, $y_{S}$, with respect to the Sphericity axis 


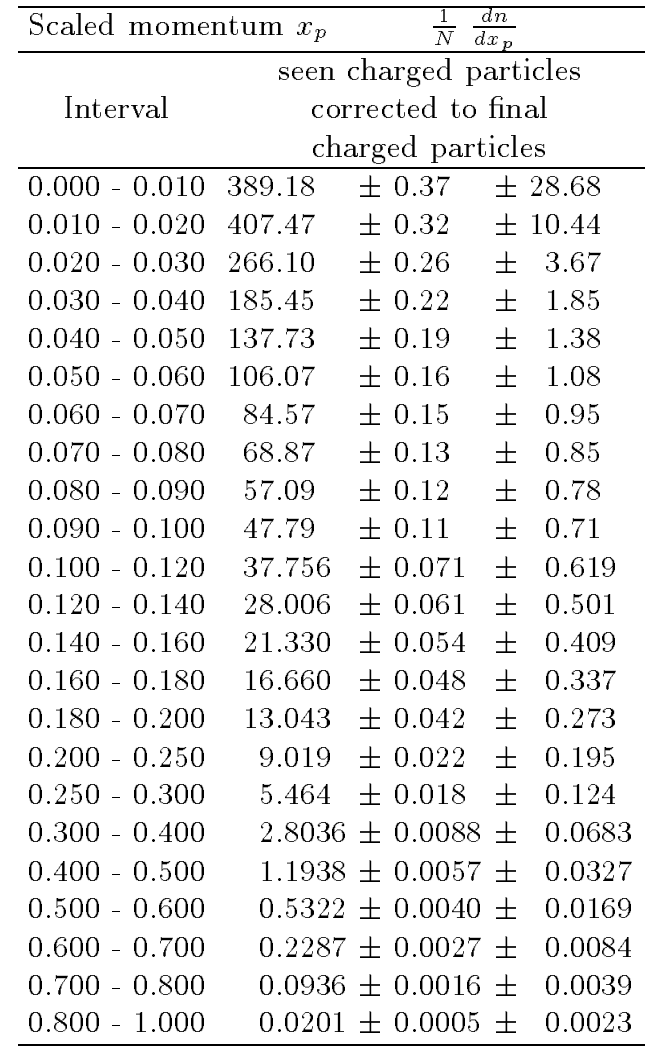

Table 17: Scaled momentum, $x_{p}$

\begin{tabular}{cc}
\hline$\xi_{p}=\ln \left(\frac{1}{x_{p}}\right)$ & $\frac{1}{N} \frac{d n}{d \xi_{p}}$ \\
\hline Interval & $\begin{array}{c}\text { seen charged particles } \\
\text { corrected to final } \\
\text { charged particles }\end{array}$ \\
\hline $0.000-0.200$ & $0.0151 \pm 0.0004 \pm 0.0022$ \\
$0.200-0.400$ & $0.0774 \pm 0.0011 \pm 0.0051$ \\
$0.400-0.600$ & $0.1987 \pm 0.0018 \pm 0.0083$ \\
$0.600-0.800$ & $0.3970 \pm 0.0023 \pm 0.0116$ \\
$0.800-1.000$ & $0.6805 \pm 0.0031 \pm 0.0161$ \\
$1.000-1.200$ & $1.0520 \pm 0.0038 \pm 0.0226$ \\
$1.200-1.400$ & $1.5128 \pm 0.0046 \pm 0.0311$ \\
$1.400-1.600$ & $2.0270 \pm 0.0053 \pm 0.0398$ \\
$1.600-1.800$ & $2.5997 \pm 0.0060 \pm 0.0481$ \\
$1.800-2.000$ & $3.1993 \pm 0.0066 \pm 0.0553$ \\
$2.000-2.200$ & $3.8069 \pm 0.0071 \pm 0.0610$ \\
$2.200-2.400$ & $4.3851 \pm 0.0076 \pm 0.0651$ \\
$2.400-2.600$ & $4.9235 \pm 0.0080 \pm 0.0680$ \\
$2.600-2.800$ & $5.4160 \pm 0.0083 \pm 0.0702$ \\
$2.800-3.000$ & $5.8079 \pm 0.0086 \pm 0.0716$ \\
$3.000-3.200$ & $6.1608 \pm 0.0088 \pm 0.0734$ \\
$3.200-3.400$ & $6.4026 \pm 0.0090 \pm 0.0753$ \\
$3.400-3.600$ & $6.5371 \pm 0.0091 \pm 0.0779$ \\
$3.600-3.800$ & $6.5822 \pm 0.0091 \pm 0.0819$ \\
$3.800-4.000$ & $6.4738 \pm 0.0090 \pm 0.0869$ \\
$4.000-4.200$ & $6.2383 \pm 0.0089 \pm 0.0933$ \\
$4.200-4.400$ & $5.8490 \pm 0.0087 \pm 0.1003$ \\
$4.400-4.600$ & $5.3231 \pm 0.0084 \pm 0.1068$ \\
$4.600-4.800$ & $4.6603 \pm 0.0079 \pm 0.1098$ \\
$4.800-5.000$ & $3.9029 \pm 0.0073 \pm 0.1064$ \\
$5.000-5.200$ & $3.1150 \pm 0.0066 \pm 0.0952$ \\
$5.200-5.400$ & $2.3781 \pm 0.0058 \pm 0.0777$ \\
\hline &
\end{tabular}

Table 18: $\xi_{p}=\ln \left(\frac{1}{x_{p}}\right)$ 


\begin{tabular}{cc}
\hline$x_{p}$ & $\left\langle p_{t}^{\text {out }}\right\rangle[\mathrm{GeV}]$ \\
\hline Interval & $\begin{array}{c}\text { seen charged particles } \\
\text { corrected to final } \\
\text { charged particles }\end{array}$ \\
\hline $0.000-0.010$ & $0.11124 \pm 0.00012 \pm 0.00097$ \\
$0.010-0.020$ & $0.19506 \pm 0.00014 \pm 0.00113$ \\
$0.020-0.030$ & $0.23694 \pm 0.00019 \pm 0.00118$ \\
$0.030-0.040$ & $0.25986 \pm 0.00023 \pm 0.00130$ \\
$0.040-0.050$ & $0.27485 \pm 0.00028 \pm 0.00137$ \\
$0.050-0.060$ & $0.28678 \pm 0.00032 \pm 0.00143$ \\
$0.060-0.070$ & $0.29812 \pm 0.00037 \pm 0.00149$ \\
$0.070-0.080$ & $0.30653 \pm 0.00041 \pm 0.00153$ \\
$0.080-0.090$ & $0.31324 \pm 0.00046 \pm 0.00157$ \\
$0.090-0.100$ & $0.31969 \pm 0.00051 \pm 0.00160$ \\
$0.100-0.120$ & $0.32899 \pm 0.00041 \pm 0.00164$ \\
$0.120-0.140$ & $0.34140 \pm 0.00049 \pm 0.00171$ \\
$0.140-0.160$ & $0.35107 \pm 0.00058 \pm 0.00176$ \\
$0.160-0.180$ & $0.35833 \pm 0.00066 \pm 0.00179$ \\
$0.180-0.200$ & $0.37165 \pm 0.00076 \pm 0.00186$ \\
$0.200-0.250$ & $0.38189 \pm 0.00059 \pm 0.00191$ \\
$0.250-0.300$ & $0.40047 \pm 0.00078 \pm 0.00200$ \\
$0.300-0.400$ & $0.41720 \pm 0.00079 \pm 0.00223$ \\
$0.400-0.500$ & $0.4348 \pm 0.0012 \pm 0.0031$ \\
$0.500-0.600$ & $0.4436 \pm 0.0019 \pm 0.0044$ \\
$0.600-0.700$ & $0.4412 \pm 0.0031 \pm 0.0062$ \\
$0.700-0.800$ & $0.4525 \pm 0.0046 \pm 0.0093$ \\
$0.800-1.000$ & $0.3778 \pm 0.0051 \pm 0.0112$ \\
\hline
\end{tabular}

Table 19: $<p_{t}^{\text {out }}>$ vs. $x_{p}$

\begin{tabular}{|c|c|}
\hline$x_{p}$ & $\left\langle p_{t}\right\rangle[\mathrm{GeV}]$ \\
\hline Interval & $\begin{array}{l}\text { seen charged particles } \\
\text { corrected to final } \\
\text { charged particles }\end{array}$ \\
\hline $0.000-0.010$ & $0.18791 \pm 0.00016 \pm 0.00171$ \\
\hline $0.010-0.020$ & $0.36450 \pm 0.00019 \pm 0.00214$ \\
\hline $0.020-0.030$ & $0.48985 \pm 0.00027 \pm 0.00245$ \\
\hline $0.030-0.040$ & $0.57682 \pm 0.00035 \pm 0.00288$ \\
\hline $0.040-0.050$ & $0.64402 \pm 0.00043 \pm 0.00322$ \\
\hline $0.050-0.060$ & $0.69974 \pm 0.00051 \pm 0.00350$ \\
\hline $0.060-0.070$ & $0.75195 \pm 0.00059 \pm 0.00376$ \\
\hline $0.070-0.080$ & $0.79370 \pm 0.00067 \pm 0.00397$ \\
\hline $0.080-0.090$ & $0.83170 \pm 0.00075 \pm 0.00416$ \\
\hline $0.090-0.100$ & $0.85958 \pm 0.00083 \pm 0.00430$ \\
\hline $0.100-0.120$ & $0.90697 \pm 0.00069 \pm 0.00453$ \\
\hline $0.120-0.140$ & $0.96796 \pm 0.00083 \pm 0.00484$ \\
\hline $0.140-0.160$ & $1.01237 \pm 0.00098 \pm 0.00506$ \\
\hline $0.160-0.180$ & $1.0555 \pm 0.0011 \pm 0.0053$ \\
\hline $0.180-0.200$ & $1.0903 \pm 0.0013 \pm 0.0055$ \\
\hline $0.200-0.250$ & $1.1594 \pm 0.0010 \pm 0.0061$ \\
\hline $0.250-0.300$ & $1.2363 \pm 0.0014 \pm 0.0076$ \\
\hline $0.300-0.400$ & $1.3226 \pm 0.0014 \pm 0.0100$ \\
\hline $0.400-0.500$ & $1.4043 \pm 0.0022 \pm 0.0135$ \\
\hline $0.500-0.600$ & $1.4327 \pm 0.0035 \pm 0.0183$ \\
\hline $0.600-0.700$ & $1.4210 \pm 0.0056 \pm 0.0246$ \\
\hline $0.700-0.800$ & $1.4134 \pm 0.0084 \pm 0.0336$ \\
\hline $0.800-1.000$ & $1.226 \pm 0.011$ \\
\hline
\end{tabular}

Table 20: $<p_{t}>$ vs. $x_{p}$ 


\section{C.2 Tables of Event Shape Distributions}

\begin{tabular}{|c|c|c|}
\hline 1-Thrust & & $\frac{d N}{(1-T)}$ \\
\hline Interval & $\begin{array}{l}\text { seen charged particles } \\
\text { corrected to final } \\
\text { charged particles }\end{array}$ & $\begin{array}{l}\text { seen charged plus neutral particles } \\
\text { corrected to final } \\
\text { charged plus neutral particles }\end{array}$ \\
\hline $0.000-0.010$ & $3.386 \pm 0.028 \pm 0.132$ & $1.030 \pm 0.019 \pm 0.076$ \\
\hline $0.010-0.020$ & $13.901 \pm 0.057 \pm 0.249$ & $10.951 \pm 0.051 \pm 0.527$ \\
\hline $0.020-0.030$ & $15.733 \pm 0.061 \pm 0.157$ & $17.645 \pm 0.066 \pm 0.547$ \\
\hline $0.030-0.040$ & $12.535 \pm 0.055 \pm 0.125$ & $14.192 \pm 0.061 \pm 0.292$ \\
\hline $0.040-0.050$ & $9.494 \pm 0.048 \pm 0.095$ & $10.009 \pm 0.050 \pm 0.152$ \\
\hline $0.050-0.060$ & $7.248 \pm 0.042 \pm 0.072$ & $7.572 \pm 0.044 \pm$ \\
\hline $0.060-0.070$ & $5.665 \pm 0.037 \pm 0.057$ & $5.760 \pm 0.038 \pm 0.076$ \\
\hline $0.070-0.080$ & $4.544 \pm 0.033 \pm 0.045$ & $4.619 \pm 0.034 \pm 0.062$ \\
\hline $0.080-0.090$ & $3.722 \pm 0.030 \pm 0.040$ & $3.792 \pm 0.031 \pm 0.051$ \\
\hline $0.090-0.100$ & $3.085 \pm 0.027 \pm 0.038$ & $3.176 \pm 0.028 \pm 0.042$ \\
\hline $0.100-0.120$ & $2.413 \pm 0.017 \pm 0.027$ & $2.456 \pm 0.018 \pm 0.032$ \\
\hline $0.120-0.140$ & $1.800 \pm 0.015 \pm 0.018$ & $1.825 \pm 0.015 \pm 0.022$ \\
\hline $0.140-0.160$ & $1.387 \pm 0.013 \pm 0.014$ & $1.401 \pm 0.013 \pm 0.016$ \\
\hline $0.160-0.180$ & $1.074 \pm 0.011 \pm 0.011$ & $1.074 \pm 0.011 \pm 0.011$ \\
\hline $0.180-0.200$ & $0.8362 \pm 0.0099 \pm 0.0096$ & $0.8262 \pm 0.0100 \pm 0.0083$ \\
\hline $0.200-0.250$ & $0.5683 \pm 0.0051 \pm 0.0101$ & $0.5525 \pm 0.0051 \pm 0.0065$ \\
\hline $0.250-0.300$ & $0.3138 \pm 0.0038 \pm 0.0077$ & $0.3030 \pm 0.0038 \pm 0.0058$ \\
\hline $0.300-0.350$ & $0.1286 \pm 0.0024 \pm 0.0045$ & $0.1312 \pm 0.0025 \pm 0.0044$ \\
\hline $0.350-0.400$ & $0.0180 \pm 0.0009 \pm 0.0011$ & $0.0238 \pm 0.0012 \pm 0.0014$ \\
\hline $0.400-0.500$ & $0.0004 \pm 0.0001 \pm 0.0001$ & $0.0007 \pm 0.0002 \pm 0.0001$ \\
\hline
\end{tabular}

Table 21: 1-Thrust, $(1-T)$

\begin{tabular}{ccccccc}
\hline Major & \multicolumn{9}{c}{$\frac{1}{N} \frac{d N}{d M}$} \\
\hline Interval & \multicolumn{3}{c}{$\begin{array}{c}\text { seen charged particles } \\
\text { corrected to final } \\
\text { charged particles }\end{array}$} & $\begin{array}{c}\text { seen charged plus neutral particles } \\
\text { corrected to final } \\
\text { charged plus neutral particles }\end{array}$ \\
\hline $0.000-0.020$ & $0.00207 \pm 0.00087 \pm 0.00313$ & 0.00040 & \pm 0.00090 & \pm 0.00005 \\
$0.020-0.040$ & 0.2165 & \pm 0.0053 & \pm 0.0114 & 0.0590 & \pm 0.0030 & \pm 0.0058 \\
$0.040-0.050$ & 1.300 & \pm 0.018 & \pm 0.013 & 0.642 & \pm 0.013 & \pm 0.028 \\
$0.050-0.060$ & 2.734 & \pm 0.027 & \pm 0.027 & 2.178 & \pm 0.024 & \pm 0.086 \\
$0.060-0.070$ & 4.245 & \pm 0.033 & \pm 0.042 & 4.303 & \pm 0.034 & \pm 0.155 \\
$0.070-0.080$ & 5.280 & \pm 0.037 & \pm 0.053 & 5.849 & \pm 0.039 & \pm 0.192 \\
$0.080-0.100$ & 6.130 & \pm 0.027 & \pm 0.061 & 6.889 & \pm 0.030 & \pm 0.194 \\
$0.100-0.120$ & 5.861 & \pm 0.027 & \pm 0.059 & 6.342 & \pm 0.028 & \pm 0.143 \\
$0.120-0.140$ & 4.881 & \pm 0.024 & \pm 0.049 & 4.890 & \pm 0.024 & \pm 0.085 \\
$0.140-0.160$ & 3.966 & \pm 0.022 & \pm 0.040 & 3.900 & \pm 0.021 & \pm 0.050 \\
$0.160-0.200$ & 3.024 & \pm 0.013 & \pm 0.030 & 2.960 & \pm 0.013 & \pm 0.030 \\
$0.200-0.240$ & 2.166 & \pm 0.011 & \pm 0.023 & 2.124 & \pm 0.011 & \pm 0.021 \\
$0.240-0.280$ & 1.5714 & \pm 0.0095 & \pm 0.0177 & 1.5562 & \pm 0.0095 & \pm 0.0156 \\
$0.280-0.320$ & 1.1782 & \pm 0.0082 & \pm 0.0146 & 1.1807 & \pm 0.0083 & \pm 0.0118 \\
$0.320-0.360$ & 0.8962 & \pm 0.0071 & \pm 0.0125 & 0.8693 & \pm 0.0071 & \pm 0.0087 \\
$0.360-0.400$ & 0.6771 & \pm 0.0061 & \pm 0.0106 & 0.6493 & \pm 0.0061 & \pm 0.0065 \\
$0.400-0.440$ & 0.5164 & \pm 0.0054 & \pm 0.0092 & 0.4820 & \pm 0.0052 & \pm 0.0048 \\
$0.440-0.480$ & 0.3940 & \pm 0.0047 & \pm 0.0079 & 0.3493 & \pm 0.0044 & \pm 0.0055 \\
$0.480-0.520$ & 0.2796 & \pm 0.0039 & \pm 0.0064 & 0.2497 & \pm 0.0037 & \pm 0.0065 \\
$0.520-0.560$ & 0.1835 & \pm 0.0031 & \pm 0.0047 & 0.1489 & \pm 0.0028 & \pm 0.0058 \\
$0.560-0.600$ & 0.1048 & \pm 0.0023 & \pm 0.0030 & 0.0714 & \pm 0.0019 & \pm 0.0038 \\
$0.600-0.640$ & 0.0453 & \pm 0.0015 & \pm 0.0015 & 0.0203 & \pm 0.0010 & \pm 0.0014 \\
\hline
\end{tabular}

Table 22: Major, $M$ 


\begin{tabular}{|c|c|c|}
\hline Minor & $\frac{1}{N} \frac{d]}{d v}$ & \\
\hline Interval & $\begin{array}{l}\text { seen charged particles } \\
\text { corrected to final } \\
\text { charged particles }\end{array}$ & $\begin{array}{l}\text { seen charged plus neutral particles } \\
\text { corrected to final } \\
\text { charged plus neutral particles }\end{array}$ \\
\hline $0.000-0.020$ & $0.1449 \pm 0.0043 \pm 0.0107$ & $0.0156 \pm 0.0017 \pm 0.0036$ \\
\hline $0.020-0.040$ & $3.109 \pm 0.019 \pm 0.031$ & $1.236 \pm 0.013 \pm 0.066$ \\
\hline $0.040-0.050$ & $7.968 \pm 0.044 \pm 0.080$ & $5.706 \pm 0.037 \pm 0.073$ \\
\hline $0.050-0.060$ & $10.353 \pm 0.050 \pm 0.104$ & $9.714 \pm 0.048 \pm 0.125$ \\
\hline $0.060-0.070$ & $11.272 \pm 0.052 \pm 0.113$ & $12.015 \pm 0.054 \pm 0.155$ \\
\hline $0.070-0.080$ & $10.890 \pm 0.051 \pm 0.109$ & $12.437 \pm 0.055 \pm 0.161$ \\
\hline $0.080-0.100$ & $9.104 \pm 0.033 \pm 0.091$ & $10.404 \pm 0.036 \pm 0.136$ \\
\hline $0.100-0.120$ & $6.292 \pm 0.027 \pm 0.063$ & $6.918 \pm 0.029 \pm$ \\
\hline $0.120-0.140$ & $4.029 \pm 0.022 \pm 0.048$ & $4.250 \pm 0.023 \pm 0.058$ \\
\hline $0.140-0.160$ & $2.519 \pm 0.017 \pm 0.037$ & $2.517 \pm 0.017 \pm 0.035$ \\
\hline $0.160-0.200$ & $1.2832 \pm 0.0085 \pm 0.0243$ & $1.2561 \pm 0.0086 \pm 0.0187$ \\
\hline $0.200-0.240$ & $0.5255 \pm 0.0055 \pm 0.0130$ & $0.4895 \pm 0.0054 \pm 0.0080$ \\
\hline $0.240-0.280$ & $0.2160 \pm 0.0035 \pm 0.0067$ & $0.2112 \pm 0.0036 \pm 0.0039$ \\
\hline $0.280-0.320$ & $0.0928 \pm 0.0023 \pm 0.0066$ & $0.0879 \pm 0.0023 \pm 0.0018$ \\
\hline $0.320-0.400$ & $0.0248 \pm 0.0009 \pm 0.0012$ & $0.0250 \pm 0.0009 \pm 0.0006$ \\
\hline
\end{tabular}

Table 23: Minor, $m$

\begin{tabular}{|c|c|c|}
\hline Oblateness & $\frac{1}{N} \frac{d]}{d t}$ & \\
\hline Interval & $\begin{array}{l}\text { seen charged particles } \\
\text { corrected to final } \\
\text { charged particles }\end{array}$ & $\begin{array}{l}\text { seen charged plus neutral particles } \\
\text { corrected to final } \\
\text { charged plus neutral particles }\end{array}$ \\
\hline $0.000-0.020$ & $7.415 \pm 0.031 \pm 0.106$ & $9.357 \pm 0.036 \pm 0.178$ \\
\hline $0.020-0.040$ & $10.840 \pm 0.037 \pm 0.108$ & $11.508 \pm 0.038 \pm 0.140$ \\
\hline $0.040-0.060$ & $7.800 \pm 0.030 \pm 0.078$ & $7.215 \pm 0.029 \pm 0$ \\
\hline $0.060-0.080$ & $5.273 \pm 0.025 \pm 0.053$ & $4.736 \pm 0.023 \pm 0.047$ \\
\hline $0.080-0.100$ & $3.797 \pm 0.021 \pm 0.038$ & $3.477 \pm 0.020 \pm 0.035$ \\
\hline $0.100-0.120$ & $2.838 \pm 0.018 \pm 0.028$ & $2.696 \pm 0.018 \pm$ \\
\hline $0.120-0.140$ & $2.218 \pm 0.016 \pm 0.022$ & $2.106 \pm 0.016 \pm 0.021$ \\
\hline $0.140-0.160$ & $1.760 \pm 0.014 \pm 0.018$ & $1.690 \pm 0.014 \pm 0.017$ \\
\hline $0.160-0.200$ & $1.3199 \pm 0.0087 \pm 0.0132$ & $1.2648 \pm 0.0085 \pm$ \\
\hline $0.200-0.240$ & $0.8874 \pm 0.0070 \pm 0.0089$ & $0.8403 \pm 0.0069 \pm 0.0087$ \\
\hline $0.240-0.280$ & $0.6208 \pm 0.0058 \pm 0.0078$ & $0.5674 \pm 0.0056 \pm 0.0065$ \\
\hline $0.280-($ & $0.4354 \pm 0.0049 \pm 0.0055$ & $0.3842 \pm 0.0046 \pm$ \\
\hline $0.320-0.360$ & $0.2959 \pm 0.0040 \pm 0.0030$ & $0.2573 \pm 0.0037 \pm 0.0043$ \\
\hline $0.360-0.400$ & $0.1987 \pm 0.0033 \pm 0.0021$ & $0.1594 \pm 0.0029 \pm 0.0037$ \\
\hline $0.400-0.440$ & $0.1163 \pm 0.0025 \pm 0.0032$ & $0.0836 \pm 0.0020 \pm 0.0030$ \\
\hline $0.440-0.520$ & $0.0453 \pm 0.0011 \pm 0.0007$ & $0.0221 \pm 0.0007 \pm 0.0015$ \\
\hline
\end{tabular}

Table 24: Oblateness, $O$ 


\begin{tabular}{crccccc}
\hline Sphericity & \multicolumn{7}{c}{$\frac{1}{N} \frac{d N}{d S}$} \\
\hline Interval & \multicolumn{9}{c}{$\begin{array}{c}\text { coen charged particles } \\
\text { charged particles }\end{array}$} & \multicolumn{3}{c}{ seen charged plus neutral particles } \\
& \multicolumn{1}{c}{ charged plus neutral particles } \\
\hline $0.000-0.010$ & 17.598 & \pm 0.068 & \pm 0.176 & 16.198 & \pm 0.067 & \pm 0.208 \\
$0.010-0.020$ & 18.570 & \pm 0.068 & \pm 0.186 & 20.008 & \pm 0.072 & \pm 0.246 \\
$0.020-0.030$ & 12.129 & \pm 0.053 & \pm 0.121 & 12.896 & \pm 0.056 & \pm 0.153 \\
$0.030-0.040$ & 8.225 & \pm 0.044 & \pm 0.082 & 8.237 & \pm 0.043 & \pm 0.094 \\
$0.040-0.050$ & 5.825 & \pm 0.036 & \pm 0.058 & 5.885 & \pm 0.037 & \pm 0.065 \\
$0.050-0.060$ & 4.600 & \pm 0.033 & \pm 0.046 & 4.458 & \pm 0.032 & \pm 0.048 \\
$0.060-0.080$ & 3.274 & \pm 0.019 & \pm 0.033 & 3.272 & \pm 0.019 & \pm 0.034 \\
$0.080-0.100$ & 2.309 & \pm 0.016 & \pm 0.023 & 2.290 & \pm 0.016 & \pm 0.023 \\
$0.100-0.120$ & 1.692 & \pm 0.014 & \pm 0.017 & 1.699 & \pm 0.014 & \pm 0.017 \\
$0.120-0.160$ & 1.2001 & \pm 0.0082 & \pm 0.0132 & 1.2018 & \pm 0.0082 & \pm 0.0120 \\
$0.160-0.200$ & 0.8053 & \pm 0.0067 & \pm 0.0104 & 0.7988 & \pm 0.0067 & \pm 0.0080 \\
$0.200-0.250$ & 0.5688 & \pm 0.0050 & \pm 0.0084 & 0.5610 & \pm 0.0050 & \pm 0.0063 \\
$0.250-0.300$ & 0.4031 & \pm 0.0043 & \pm 0.0065 & 0.3926 & \pm 0.0042 & \pm 0.0051 \\
$0.300-0.350$ & 0.2931 & \pm 0.0036 & \pm 0.0049 & 0.2810 & \pm 0.0035 & \pm 0.0043 \\
$0.350-0.400$ & 0.2206 & \pm 0.0031 & \pm 0.0035 & 0.2099 & \pm 0.0030 & \pm 0.0037 \\
$0.400-0.500$ & 0.1528 & \pm 0.0018 & \pm 0.0025 & 0.1441 & \pm 0.0018 & \pm 0.0032 \\
$0.500-0.600$ & 0.0886 & \pm 0.0014 & \pm 0.0021 & 0.0842 & \pm 0.0013 & \pm 0.0023 \\
$0.600-0.700$ & 0.04319 & \pm 0.00094 & \pm 0.00209 & 0.04160 & \pm 0.00092 & \pm 0.00129 \\
$0.700-0.850$ & 0.00778 & \pm 0.00032 & \pm 0.0008 & 0.00758 & \pm 0.00032 & \pm 0.00024 \\
\hline
\end{tabular}

Table 25: Sphericity, $S$

\begin{tabular}{ccccccc}
\hline Aplanarity & \multicolumn{7}{c}{$\frac{1}{N} \frac{d N}{d A}$} \\
Interval & \multicolumn{3}{c}{$\begin{array}{c}\text { seen charged particles } \\
\text { corrected to final } \\
\text { charged particles }\end{array}$} & \multicolumn{3}{c}{$\begin{array}{c}\text { seen charged plus neutral particles } \\
\text { charged plus neutral particles }\end{array}$} \\
\hline $0.000-0.005$ & 81.41 & \pm 0.20 & \pm 0.81 & 75.10 & \pm 0.19 & \pm 0.75 \\
$0.005-0.010$ & 49.12 & \pm 0.15 & \pm 0.49 & 55.31 & \pm 0.17 & \pm 0.55 \\
$0.010-0.015$ & 24.05 & \pm 0.11 & \pm 0.24 & 26.03 & \pm 0.11 & \pm 0.28 \\
$0.015-0.020$ & 13.668 & \pm 0.079 & \pm 0.137 & 13.927 & \pm 0.079 & \pm 0.176 \\
$0.020-0.030$ & 6.893 & \pm 0.039 & \pm 0.088 & 6.768 & \pm 0.038 & \pm 0.098 \\
$0.030-0.040$ & 3.270 & \pm 0.027 & \pm 0.057 & 3.014 & \pm 0.025 & \pm 0.056 \\
$0.040-0.060$ & 1.443 & \pm 0.013 & \pm 0.032 & 1.281 & \pm 0.012 & \pm 0.035 \\
$0.060-0.080$ & 0.5841 & \pm 0.0080 & \pm 0.0153 & 0.5181 & \pm 0.0075 & \pm 0.0188 \\
$0.080-0.100$ & 0.2825 & \pm 0.0056 & \pm 0.0085 & 0.2619 & \pm 0.0054 & \pm 0.0118 \\
$0.100-0.120$ & 0.1515 & \pm 0.0041 & \pm 0.0051 & 0.1461 & \pm 0.0041 & \pm 0.0079 \\
$0.120-0.140$ & 0.0831 & \pm 0.0030 & \pm 0.0031 & 0.0758 & \pm 0.0029 & \pm 0.0043 \\
$0.140-0.160$ & 0.0535 & \pm 0.0026 & \pm 0.0023 & 0.0467 & \pm 0.0023 & \pm 0.0027 \\
$0.160-0.200$ & 0.0262 & \pm 0.0012 & \pm 0.0014 & 0.0234 & \pm 0.0011 & \pm 0.0014 \\
$0.200-0.250$ & 0.00969 & \pm 0.00067 & \pm 0.00062 & 0.00884 & \pm 0.00061 & \pm 0.00052 \\
$0.250-0.300$ & $0.00320 \pm 0.00037$ & \pm 0.00075 & 0.00310 & \pm 0.00040 & \pm 0.00018 \\
\hline
\end{tabular}

Table 26: Aplanarity, $A$ 


\begin{tabular}{|c|c|c|}
\hline Planarity & $\frac{1}{N} \frac{d]}{d}$ & \\
\hline Interval & $\begin{array}{l}\text { seen charged particles } \\
\text { corrected to final } \\
\text { charged particles }\end{array}$ & $\begin{array}{l}\text { seen charged plus neutral particles } \\
\text { corrected to final } \\
\text { charged plus neutral particles }\end{array}$ \\
\hline $0.000-0.005$ & $\pm 0.18 \quad \pm 0.65$ & $68.69 \pm 0.19$ \\
\hline $0.005-0.010$ & \pm 0.12 & \pm 0.12 \\
\hline $0.010-0.015$ & $18.177 \pm 0.092 \pm 0.182$ & $17.091 \pm 0.088 \pm 0.188$ \\
\hline $0.015-0.020$ & $12.001 \pm 0.075 \pm 0.120$ & $11.370 \pm 0.072 \pm 0.127$ \\
\hline $0.020-0.025$ & $8.607 \pm 0.062 \pm 0.086$ & $8.417 \pm 0.062 \pm 0.095$ \\
\hline $0.025-0.030$ & $6.792 \pm 0.056 \pm 0.068$ & $6.578 \pm 0.055 \pm 0.075$ \\
\hline $0.030-0.035$ & $5.542 \pm 0.051 \pm 0.055$ & $5.479 \pm 0.050 \pm 0.063$ \\
\hline $0.035-0.040$ & $4.519 \pm 0.045 \pm 0.045$ & $4.493 \pm 0.045 \pm$ \\
\hline $0.040-0.050$ & $3.640 \pm 0.029 \pm 0.036$ & $3.610 \pm 0.029 \pm 0.042$ \\
\hline $0.050-0.060$ & $2.757 \pm 0.025 \pm 0.028$ & $2.749 \pm 0.025 \pm 0.033$ \\
\hline $0.060-0.080$ & $1.986 \pm 0.015 \pm 0.022$ & $1.987 \pm 0.015 \pm 0.024$ \\
\hline $0.080-0.100$ & $1.362 \pm 0.012 \pm 0.017$ & $1.362 \pm 0.012 \pm 0.017$ \\
\hline $0.100-0.120$ & $1.008 \pm 0.011 \pm 0.014$ & $1.008 \pm 0.011 \pm 0.013$ \\
\hline $0.120-0.160$ & $0.6777 \pm 0.0061 \pm 0.0107$ & $0.6676 \pm 0.0061 \pm 0.0093$ \\
\hline $0.160-0.200$ & $0.4477 \pm 0.0050 \pm 0.0083$ & $0.4248 \pm 0.0048 \pm 0.0063$ \\
\hline $0.200-0.250$ & $0.2755 \pm 0.0034 \pm 0.0060$ & $0.2692 \pm 0.0034 \pm 0.0042$ \\
\hline $0.250-0.300$ & $0.1801 \pm 0.0028 \pm 0.0045$ & $0.1742 \pm 0.0028 \pm 0.0029$ \\
\hline $0.300-0.350$ & $0.1114 \pm 0.0022 \pm 0.0032$ & $0.1042 \pm 0.0021 \pm 0.0019$ \\
\hline $0.350-0.400$ & $0.0575 \pm 0.0015 \pm 0.0018$ & $0.0566 \pm 0.0015 \pm 0.0011$ \\
\hline $0.400-0.500$ & $0.0137 \pm 0.0005 \pm 0.0005$ & $0.0145 \pm 0.0006 \pm 0.0003$ \\
\hline
\end{tabular}

Table 27: Planarity, $P$

\begin{tabular}{|c|c|c|}
\hline C-Parameter & $\frac{1}{N} \frac{d d}{d t}$ & \\
\hline Interval & $\begin{array}{l}\text { seen charged particles } \\
\text { corrected to final } \\
\text { charged particles }\end{array}$ & $\begin{array}{l}\text { seen charged plus neutral particles } \\
\text { corrected to final } \\
\text { charged plus neutral particles }\end{array}$ \\
\hline $0.000-0.040$ & $0.3806 \pm 0.0047 \pm 0.0140$ & $0.0881 \pm 0.0030 \pm 0.0067$ \\
\hline $0.040-0.080$ & $2.388 \pm 0.012 \pm 0.050$ & $1.5383 \pm 0.0100 \pm 0.0831$ \\
\hline $0.080-0.120$ & $3.653 \pm 0.015 \pm 0.042$ & $3.909 \pm 0.016 \pm 0.142$ \\
\hline $0.120-0.160$ & $3.323 \pm 0.014 \pm 0.033$ & $3.833 \pm 0.016 \pm 0.088$ \\
\hline $0.160-0.200$ & $2.637 \pm 0.013 \pm 0.026$ & $2.835 \pm 0.013 \pm 0.040$ \\
\hline $0.200-0.240$ & $2.091 \pm 0.011 \pm 0.021$ & $2.164 \pm 0.012 \pm 0.022$ \\
\hline $0.240-0.280$ & $1.680 \pm 0.010 \pm 0.017$ & $1.716 \pm 0.010 \pm 0.017$ \\
\hline $0.280-0.320$ & $1.3757 \pm 0.0092 \pm 0.0138$ & $1.3860 \pm 0.0092 \pm 0.0139$ \\
\hline $0.320-0.360$ & $1.1545 \pm 0.0083 \pm 0.0129$ & $1.1623 \pm 0.0084 \pm 0.0116$ \\
\hline $0.360-0.400$ & $0.9709 \pm 0.0076 \pm 0.0127$ & $0.9720 \pm 0.0077 \pm 0.0097$ \\
\hline $0.400-0.440$ & $0.8100 \pm 0.0068 \pm 0.0121$ & $0.8349 \pm 0.0072 \pm 0.0083$ \\
\hline $0.440-0.480$ & $0.7162 \pm 0.0065 \pm 0.0120$ & $0.7161 \pm 0.0066 \pm 0.0072$ \\
\hline $0.480-0.520$ & $0.6247 \pm 0.0060 \pm 0.0116$ & $0.6205 \pm 0.0061 \pm 0.0062$ \\
\hline $0.520-0.560$ & $0.5573 \pm 0.0057 \pm 0.0113$ & $0.5441 \pm 0.0057 \pm 0.0054$ \\
\hline $0.560-0.600$ & $0.4939 \pm 0.0053 \pm 0.0108$ & $0.4844 \pm 0.0054 \pm 0.0050$ \\
\hline $0.600-0.640$ & $0.4245 \pm 0.0049 \pm 0.0100$ & $0.4209 \pm 0.0050 \pm 0.0063$ \\
\hline $0.640-0.680$ & $0.3860 \pm 0.0048 \pm 0.0097$ & $0.3699 \pm 0.0046 \pm 0.0079$ \\
\hline $0.680-0.720$ & $0.3589 \pm 0.0046 \pm 0.0095$ & $0.3286 \pm 0.0044 \pm 0.0099$ \\
\hline $0.720-0.760$ & $0.3063 \pm 0.0041 \pm 0.0084$ & $0.2813 \pm 0.0040 \pm 0.0129$ \\
\hline $0.760-0.800$ & $0.2672 \pm 0.0039 \pm 0.0073$ & $0.2178 \pm 0.0033 \pm 0.0151$ \\
\hline $0.800-0.840$ & $0.1485 \pm 0.0028 \pm 0.0037$ & $0.1287 \pm 0.0026 \pm 0.0130$ \\
\hline $0.840-0.880$ & $0.0716 \pm 0.0020 \pm 0.0015$ & $0.0542 \pm 0.0016 \pm 0.0076$ \\
\hline $0.880-0.920$ & $0.0307 \pm 0.0013 \pm 0.0004$ & $0.0212 \pm 0.0009 \pm 0.0040$ \\
\hline
\end{tabular}

Table 28: C-Parameter, $C$ 


\begin{tabular}{|c|c|c|}
\hline D-Parameter & $\frac{1}{N} \frac{a}{c}$ & \\
\hline Interval & $\begin{array}{l}\text { seen charged particles } \\
\text { corrected to final } \\
\text { charged particles }\end{array}$ & $\begin{array}{l}\text { seen charged plus neutral particles } \\
\text { corrected to final } \\
\text { charged plus neutral particles }\end{array}$ \\
\hline $0.000-0.008$ & $29.731 \pm 0.093 \pm 0.308$ & $22.228 \pm 0.082 \pm 0.868$ \\
\hline $0.008-0.016$ & $19.922 \pm 0.077 \pm 0.199$ & $22.766 \pm 0.085 \pm 0.440$ \\
\hline $0.016-0.030$ & $11.026 \pm 0.044 \pm 0.110$ & $12.107 \pm 0.047 \pm 0.150$ \\
\hline $0.030-0.044$ & $6.513 \pm 0.034 \pm 0.065$ & $6.879 \pm 0.035 \pm$ \\
\hline $0.044-0.066$ & $4.066 \pm 0.021 \pm 0.045$ & $4.284 \pm 0.022 \pm 0.053$ \\
\hline $0.066-0.088$ & $2.640 \pm 0.017 \pm 0.033$ & $2.727 \pm 0.018 \pm$ \\
\hline $0.088-0.112$ & $1.864 \pm 0.014 \pm 0.025$ & $1.909 \pm 0.014 \pm 0.028$ \\
\hline $0.112-0.136$ & $1.352 \pm 0.012 \pm 0.019$ & $1.415 \pm 0.012 \pm$ \\
\hline $0.136-0.162$ & $1.0013 \pm 0.0095 \pm 0.0149$ & $1.051 \pm 0.010 \pm$ \\
\hline $0.162-0.188$ & $0.7829 \pm 0.0085 \pm 0.0123$ & $0.7977 \pm 0.0089 \pm$ \\
\hline $0.188-0.218$ & $0.6187 \pm 0.0071 \pm 0.0103$ & $0.6155 \pm 0.0073 \pm$ \\
\hline $0.218-0.248$ & $0.4490 \pm 0.0059 \pm 0.0081$ & $0.4566 \pm 0.0063 \pm$ \\
\hline $0.248-0.284$ & $0.3425 \pm 0.0048 \pm 0.0068$ & $0.3341 \pm 0.0049 \pm$ \\
\hline $0.284-0.320$ & $0.2575 \pm 0.0042 \pm 0.0057$ & $0.2452 \pm 0.0042 \pm 0.0049$ \\
\hline $0.320-0.360$ & $0.1884 \pm 0.0034 \pm 0.0047$ & $0.1774 \pm 0.0033 \pm$ \\
\hline $0.360-0.400$ & $0.1363 \pm 0.0028 \pm 0.0038$ & $0.1234 \pm 0.0028 \pm 0.0028$ \\
\hline $0.400-0.450$ & $0.0962 \pm 0.0021 \pm 0.0029$ & $0.0902 \pm 0.0021 \pm 0.0023$ \\
\hline $0.450-0.500$ & $0.0644 \pm 0.0017 \pm 0.0021$ & $0.0603 \pm 0.0017 \pm 0.0018$ \\
\hline $0.500-0.560$ & $0.0394 \pm 0.0012 \pm 0.0014$ & $0.0368 \pm 0.0012 \pm 0.0013$ \\
\hline $0.560-0.620$ & $0.0277 \pm 0.0010 \pm 0.0010$ & $0.0222 \pm 0.0009 \pm 0.0009$ \\
\hline $0.620-0.710$ & $0.0151 \pm 0.0006 \pm 0.0006$ & $0.0128 \pm 0.0006 \pm 0.0006$ \\
\hline $0.710-0.800$ & $0.0071 \pm 0.0004 \pm 0.0003$ & $0.0052 \pm 0.0004 \pm 0.0003$ \\
\hline
\end{tabular}

Table 29: D-Parameter, $D$

\begin{tabular}{|c|c|c|}
\hline \multicolumn{3}{|c|}{ Heavy Hemisphere Mass } \\
\hline Interval & $\begin{array}{l}\text { seen charged particles } \\
\text { corrected to final } \\
\text { charged particles }\end{array}$ & $\begin{array}{l}\text { seen charged plus neutral particles } \\
\text { corrected to final } \\
\text { charged plus neutral particles }\end{array}$ \\
\hline $0.000-0.010$ & $4.450 \pm 0.032 \pm 0.260$ & $1.994 \pm 0.027 \pm 0.166$ \\
\hline $0.010-0.020$ & $17.310 \pm 0.062 \pm 0.173$ & $18.580 \pm 0.065 \pm 0.709$ \\
\hline $0.020-0.030$ & $17.290 \pm 0.066 \pm 0.267$ & $20.678 \pm 0.076 \pm 0$ \\
\hline $0.030-0.040$ & $12.996 \pm 0.058 \pm 0.206$ & $13.377 \pm 0.060 \pm 0$ \\
\hline $0.040-0.050$ & $9.496 \pm 0.050 \pm 0.154$ & $8.965 \pm 0.049 \pm$ \\
\hline $0.050-0.060$ & $6.957 \pm 0.043 \pm 0.116$ & $6.558 \pm 0.041 \pm$ \\
\hline $0.060-0.080$ & $4.811 \pm 0.025 \pm 0.083$ & $4.515 \pm 0.024 \pm 0.082$ \\
\hline $0.080-0.100$ & $3.064 \pm 0.020 \pm 0.055$ & $2.914 \pm 0.019 \pm 0.037$ \\
\hline $0.100-0.120$ & $2.109 \pm 0.016 \pm 0.039$ & $1.991 \pm 0.016 \pm 0.020$ \\
\hline $0.120-0.140$ & $1.468 \pm 0.014 \pm 0.028$ & $1.406 \pm 0.013 \pm 0.014$ \\
\hline $0.140-0.160$ & $1.078 \pm 0.012 \pm 0.021$ & $1.010 \pm 0.011 \pm 0.010$ \\
\hline $0.160-0.200$ & $0.6959 \pm 0.0067 \pm 0.0142$ & $0.6319 \pm 0.0063 \pm 0.0063$ \\
\hline $0.200-0.250$ & $0.3444 \pm 0.0042 \pm 0.0074$ & $0.3085 \pm 0.0039 \pm 0.0051$ \\
\hline $0.250-0.300$ & $0.1497 \pm 0.0027 \pm 0.0033$ & $0.1115 \pm 0.0022 \pm 0.0039$ \\
\hline $0.300-0.350$ & $0.0547 \pm 0.0015 \pm 0.0012$ & $0.0184 \pm 0.0008 \pm 0.0012$ \\
\hline $0.350-0.400$ & $0.0192 \pm 0.0009 \pm 0.0004$ & $0.0008 \pm 0.0002 \pm 0.0001$ \\
\hline
\end{tabular}

Table 30: Heavy Hemisphere Mass, $M_{h i g h}^{2} / E_{v i s}^{2}$ 


\begin{tabular}{|c|c|c|}
\hline Light Hemisp & ere Mass & $\frac{d N}{d M_{\text {low }}^{2} / E_{v i s}^{2}}$ \\
\hline Interval & $\begin{array}{l}\text { seen charged particles } \\
\text { corrected to final } \\
\text { charged particles }\end{array}$ & $\begin{array}{l}\text { seen charged plus neutral particles } \\
\text { corrected to final } \\
\text { charged plus neutral particles }\end{array}$ \\
\hline $0.000-0.010$ & $39.362 \pm 0.093 \pm 1.220$ & $23.414 \pm 0.074 \pm 1.595$ \\
\hline $0.010-0.020$ & $31.177 \pm 0.092 \pm 1.074$ & \pm 0.11 \\
\hline $0.020-0.030$ & $13.733 \pm 0.065 \pm 0.523$ & $18.080 \pm 0.081 \pm$ \\
\hline $0.030-0.040$ & $6.486 \pm 0.044 \pm 0.270$ & $7.704 \pm 0.052 \pm$ \\
\hline $0.040-0.050$ & $3.358 \pm 0.032 \pm 0.147$ & $3.922 \pm 0.036 \pm 0$ \\
\hline $0.050-0$ & $1.879 \pm 0.024 \pm 0.082$ & $2.128 \pm 0.026 \pm 0$ \\
\hline $0.060-0.080$ & $0.901 \pm 0.012 \pm 0.040$ & $1.013 \pm 0.013 \pm$ \\
\hline $0.080-0.100$ & $0.3212 \pm 0.0072 \pm 0.0175$ & $0.3748 \pm 0.0079 \pm 0.0241$ \\
\hline $0.100-0.120$ & $0.1087 \pm 0.0044 \pm 0.0082$ & $0.1412 \pm 0.0050 \pm 0.0089$ \\
\hline
\end{tabular}

Table 31: Light Hemisphere Mass, $M_{\text {low }}^{2} / E_{v i s}^{2}$

\begin{tabular}{|c|c|c|}
\hline \multicolumn{3}{|c|}{ Difference of the Hemisphere Masses $\frac{1}{N} \frac{d N}{d M_{d i f f}^{2} / E_{v i s}^{2}}$} \\
\hline Interval & $\begin{array}{l}\text { seen charged particles } \\
\text { corrected to final } \\
\text { charged particles }\end{array}$ & $\begin{array}{l}\text { seen charged plus neutral particles } \\
\text { corrected to final } \\
\text { charged plus neutral particles }\end{array}$ \\
\hline $0.000-0.010$ & $29.229 \quad \pm 0.082 \quad \pm 0.292$ & $35.393 \pm 0.092 \pm 0.354$ \\
\hline $0.010-0.020$ & $19.648 \pm 0.068 \pm 0.196$ & $20.745 \pm 0.071 \pm 0.207$ \\
\hline $0.020-0.030$ & $12.462 \pm 0.055 \pm 0.125$ & $11.426 \pm 0.052 \pm 0.114$ \\
\hline $0.030-0.040$ & $8.332 \pm 0.045 \pm 0.083$ & $7.170 \pm 0.041 \pm 0.072$ \\
\hline $0.040-0.060$ & $5.164 \pm 0.025 \pm 0.052$ & $4.344 \pm 0.023 \pm 0.043$ \\
\hline $0.060-0.080$ & $2.987 \pm 0.019 \pm 0.034$ & $2.605 \pm 0.017 \pm 0.026$ \\
\hline $0.080-0.120$ & $1.646 \pm 0.010 \pm 0.022$ & $1.4238 \pm 0.0092 \pm 0.0142$ \\
\hline $0.120-0.160$ & $0.8298 \pm 0.0070 \pm 0.0136$ & $0.7061 \pm 0.0064 \pm 0.0071$ \\
\hline $0.160-0.200$ & $0.4606 \pm 0.0053 \pm 0.0091$ & $0.3831 \pm 0.0046 \pm 0.0044$ \\
\hline $0.200-0.250$ & $0.2358 \pm 0.0033 \pm 0.0060$ & $0.1836 \pm 0.0028 \pm 0.0032$ \\
\hline $0.250-0.300$ & $0.1043 \pm 0.0022 \pm 0.0036$ & $0.0579 \pm 0.0015 \pm 0.0018$ \\
\hline $0.300-0.350$ & $0.0417 \pm 0.0013 \pm 0.0020$ & $0.0075 \pm 0.0006 \pm 0.0006$ \\
\hline $0.350-0.400$ & $0.0151 \pm 0.0008 \pm 0.0010$ & $0.0003 \pm 0.0002 \pm 0.0001$ \\
\hline
\end{tabular}

Table 32: Difference of the Hemisphere Masses, $M_{d i f f}^{2} / E_{v i s}^{2}$ 


\begin{tabular}{|c|c|c|}
\hline \multicolumn{3}{|c|}{ Wide Hemisphere Broadening } \\
\hline Interval & $\begin{array}{l}\text { seen charged particles } \\
\text { corrected to final } \\
\text { charged particles }\end{array}$ & $\begin{array}{l}\text { seen charged plus neutral particles } \\
\text { corrected to final } \\
\text { charged plus neutral particles }\end{array}$ \\
\hline $0.010-0.020$ & $2.027 \pm 0.023 \pm 0.020$ & $0.6707 \pm 0.0096 \pm 0.1077$ \\
\hline $0.020-0.030$ & $9.923 \pm 0.051 \pm 0.232$ & $7.538 \pm 0.038 \pm 0.809$ \\
\hline $0.030-0.040$ & $14.758 \pm 0.061 \pm 0.245$ & $14.690 \pm 0.058 \pm 0.745$ \\
\hline $0.040-0.050$ & $13.374 \pm 0.056 \pm 0.140$ & $13.942 \pm 0.057 \pm 0.592$ \\
\hline $0.050-0.060$ & $10.620 \pm 0.050 \pm 0.106$ & $11.298 \pm 0.053 \pm 0.379$ \\
\hline $0.060-0.070$ & $8.369 \pm 0.044 \pm 0.084$ & $9.065 \pm 0.048 \pm 0.266$ \\
\hline $0.070-0.080$ & $6.811 \pm 0.040 \pm 0.068$ & $7.387 \pm 0.043 \pm 0.222$ \\
\hline $0.080-0.100$ & $5.146 \pm 0.025 \pm 0.051$ & $5.445 \pm 0.026 \pm 0.176$ \\
\hline $0.100-0.120$ & $3.584 \pm 0.020 \pm 0.036$ & $3.796 \pm 0.022 \pm 0.127$ \\
\hline $0.120-0.140$ & $2.577 \pm 0.017 \pm 0.026$ & $2.670 \pm 0.018 \pm 0.087$ \\
\hline $0.140-0.170$ & $1.733 \pm 0.012 \pm 0.017$ & $1.756 \pm 0.012 \pm 0.051$ \\
\hline $0.170-0.200$ & $1.0540 \pm 0.0090 \pm 0.0105$ & $1.0580 \pm 0.0092 \pm 0.0218$ \\
\hline $0.200-0.240$ & $0.5388 \pm 0.0056 \pm 0.0135$ & $0.5288 \pm 0.0056 \pm 0.0053$ \\
\hline $0.240-0.280$ & $0.1710 \pm 0.0031 \pm 0.0104$ & $0.1460 \pm 0.0028 \pm 0.0071$ \\
\hline $0.280-0.320$ & $0.0262 \pm 0.0011 \pm 0.0028$ & $0.0029 \pm 0.0004 \pm 0.0003$ \\
\hline
\end{tabular}

Table 33: Wide Hemisphere Broadening, $B_{\max }$

\begin{tabular}{|c|c|c|}
\hline \multicolumn{3}{|c|}{ Narrow Hemisphere Broadening } \\
\hline Interval & $\begin{array}{l}\text { seen charged particles } \\
\text { corrected to final } \\
\text { charged particles }\end{array}$ & $\begin{array}{l}\text { seen charged plus neutral particles } \\
\text { corrected to final } \\
\text { charged plus neutral particles }\end{array}$ \\
\hline $0.000-0.010$ & $2.502 \pm 0.025 \quad \pm 0.025$ & $0.645 \pm 0.010 \pm 0.096$ \\
\hline $0.010-0.020$ & $18.823 \pm 0.068 \pm 0.188$ & $11.169 \pm 0.045 \pm 1.006$ \\
\hline $0.020-0.030$ & $28.683 \pm 0.084 \pm 0.287$ & $28.908 \pm 0.082 \pm 1.823$ \\
\hline $0.030-0.040$ & $20.703 \pm 0.071 \pm 0.207$ & $25.972 \pm 0.083 \pm 1.478$ \\
\hline $0.040-0.050$ & $11.654 \pm 0.052 \pm 0.117$ & $14.119 \pm 0.061 \pm 0.860$ \\
\hline $0.050-0.060$ & $6.558 \pm 0.039 \pm 0.066$ & $7.500 \pm 0.044 \pm 0.494$ \\
\hline $0.060-0.080$ & $3.191 \pm 0.019 \pm 0.032$ & $3.405 \pm 0.021 \pm 0.233$ \\
\hline $0.080-0.100$ & $1.295 \pm 0.012 \pm 0.026$ & $1.320 \pm 0.013 \pm 0.089$ \\
\hline $0.100-0.120$ & $0.5561 \pm 0.0078 \pm 0.0185$ & $0.5448 \pm 0.0082 \pm 0.0328$ \\
\hline $0.120-0.150$ & $0.1921 \pm 0.0037 \pm 0.0054$ & $0.1916 \pm 0.0040 \pm 0.0104$ \\
\hline $0.150-0.180$ & $0.0319 \pm 0.0015 \pm 0.0012$ & $0.0366 \pm 0.0017 \pm 0.0034$ \\
\hline
\end{tabular}

Table 34: Narrow Hemisphere Broadening, $B_{\text {min }}$ 


\begin{tabular}{|c|c|c|}
\hline \multicolumn{3}{|c|}{ Total Hemisphere Broadening } \\
\hline Interval & $\begin{array}{l}\text { seen charged particles } \\
\text { corrected to final } \\
\text { charged particles }\end{array}$ & $\begin{array}{l}\text { seen charged plus neutral particles } \\
\text { corrected to final } \\
\text { charged plus neutral particles }\end{array}$ \\
\hline $0.020-0.030$ & $0.956 \pm 0.016 \pm 0.010$ & $0.2030 \pm 0.0055 \pm 0.0383$ \\
\hline $0.030-0.040$ & $3.765 \pm 0.031 \pm 0.038$ & $1.628 \pm 0.015 \pm 0.183$ \\
\hline $0.040-0.050$ & $7.117 \pm 0.043 \pm 0.071$ & $4.999 \pm 0.031 \pm 0.463$ \\
\hline $0.050-0.060$ & $9.301 \pm 0.048 \pm 0.093$ & $8.190 \pm 0.041 \pm 0.644$ \\
\hline $0.060-0.070$ & $10.059 \pm 0.050 \pm 0.101$ & $9.887 \pm 0.047 \pm 0.661$ \\
\hline $0.070-0.080$ & $9.212 \pm 0.047 \pm 0.092$ & $9.883 \pm 0.049 \pm 0.564$ \\
\hline $0.080-0.090$ & $7.958 \pm 0.043 \pm 0.080$ & $9.007 \pm 0.047 \pm 0.443$ \\
\hline $0.090-0.100$ & $6.815 \pm 0.040 \pm 0.068$ & $7.746 \pm 0.044 \pm 0$ \\
\hline $0.100-0.110$ & $5.898 \pm 0.037 \pm 0.059$ & $6.714 \pm 0.041 \pm 0.255$ \\
\hline $0.110-0.130$ & $4.785 \pm 0.024 \pm 0.048$ & $5.393 \pm 0.026 \pm 0.180$ \\
\hline $0.130-0.150$ & $3.619 \pm 0.021 \pm 0.036$ & $3.998 \pm 0.023 \pm 0.125$ \\
\hline $0.150-0.170$ & $2.737 \pm 0.018 \pm 0.027$ & $2.980 \pm 0.019 \pm 0.098$ \\
\hline $0.170-0.190$ & $2.143 \pm 0.016 \pm 0.021$ & $2.294 \pm 0.017 \pm 0.085$ \\
\hline $0.190-0.210$ & $1.670 \pm 0.014 \pm 0.017$ & $1.747 \pm 0.015 \pm 0.075$ \\
\hline $0.210-0.240$ & $1.2151 \pm 0.0096 \pm 0.0122$ & $1.242 \pm 0.010 \pm 0.063$ \\
\hline $0.240-0.270$ & $0.8189 \pm 0.0078 \pm 0.0082$ & $0.8125 \pm 0.0080 \pm 0.0469$ \\
\hline $0.270-0.300$ & $0.5167 \pm 0.0061 \pm 0.0065$ & $0.4974 \pm 0.0062 \pm 0.0296$ \\
\hline $0.300-0.330$ & $0.2701 \pm 0.0044 \pm 0.0054$ & $0.2285 \pm 0.0041 \pm 0.0119$ \\
\hline $0.330-0.360$ & $0.0856 \pm 0.0024 \pm 0.0028$ & $0.0732 \pm 0.0024 \pm 0.0007$ \\
\hline
\end{tabular}

Table 35: Total Hemisphere Broadening, $B_{\text {sum }}$

\begin{tabular}{|c|c|c|c|c|}
\hline \multicolumn{5}{|c|}{ Difference of the Hemisphere Broadenings $\frac{1}{N} \frac{d N}{d B_{d i f f}}$} \\
\hline Interval & & $\begin{array}{l}\text { en charged } p \\
\text { corrected to } \\
\text { charged part }\end{array}$ & & $\begin{array}{l}\text { seen charged plus neutral particles } \\
\text { corrected to final } \\
\text { charged plus neutral particles }\end{array}$ \\
\hline $0.000-0.010$ & 24.778 & $\pm 0.078 \pm$ & 0.248 & $26.630 \pm 0.081 \pm 0.459$ \\
\hline $0.010-0.020$ & 18.957 & $\pm 0.068 \pm$ & 0.190 & $18.684 \pm 0.066 \pm 0.292$ \\
\hline $0.020-0.030$ & 13.050 & $\pm 0.056 \pm$ & 0.131 & $12.343 \pm 0.054 \pm 0.186$ \\
\hline $0.030-0.040$ & 9.129 & $\pm 0.046 \pm$ & 0.091 & $8.819 \pm 0.046 \pm 0.134$ \\
\hline $0.040-0.050$ & 6.689 & $\pm 0.040 \pm$ & 0.067 & $6.688 \pm 0.040 \pm 0.106$ \\
\hline $0.050-0.060$ & 5.220 & $\pm 0.035 \pm$ & 0.052 & $5.111 \pm 0.035 \pm 0.084$ \\
\hline $0.060-0.070$ & 4.087 & $\pm 0.031 \pm$ & 0.041 & $4.071 \pm 0.031 \pm 0.068$ \\
\hline $0.070-0.080$ & 3.314 & $\pm 0.028 \pm$ & 0.033 & $3.271 \pm 0.028 \pm 0.054$ \\
\hline $0.080-0.090$ & 2.677 & $\pm 0.025 \pm$ & 0.027 & $2.681 \pm 0.025 \pm 0.043$ \\
\hline $0.090-0.100$ & 2.218 & $\pm 0.023 \pm$ & 0.022 & $2.233 \pm 0.023 \pm 0.035$ \\
\hline $0.100-0.120$ & 1.662 & $\pm 0.014 \pm$ & 0.017 & $1.647 \pm 0.014 \pm 0.026$ \\
\hline $0.120-0.140$ & 1.145 & $\pm 0.012 \pm$ & 0.011 & $1.111 \pm 0.011 \pm 0.019$ \\
\hline $0.140-0.160$ & 0.8022 & $\pm 0.0098 \pm$ & 0.0108 & $0.7618 \pm 0.0095 \pm 0.0144$ \\
\hline $0.160-0.180$ & 0.5135 & $\pm 0.0078 \pm$ & 0.0109 & $0.5138 \pm 0.0078 \pm 0.0119$ \\
\hline $0.180-0.200$ & 0.3325 & $\pm 0.0063 \pm$ & 0.0091 & $0.3167 \pm 0.0062 \pm 0.0098$ \\
\hline $0.200-0.240$ & 0.1540 & $\pm 0.0029 \pm$ & 0.0053 & $0.1265 \pm 0.0026 \pm 0.0056$ \\
\hline $0.240-0.280$ & 0.0389 & $\pm 0.0014 \pm$ & 0.0023 & $0.0117 \pm 0.0008 \pm 0.0008$ \\
\hline
\end{tabular}

Table 36: Difference of the Hemisphere Broadenings, $B_{d i f f}$. 


\begin{tabular}{|c|c|c|c|}
\hline \multirow{2}{*}{$\begin{array}{c}\text { Interval } \\
0.000-0.010\end{array}$} & \multicolumn{2}{|c|}{$\begin{array}{l}\text { seen charged particles } \\
\text { corrected to final } \\
\text { charged particles } \\
\end{array}$} & $\begin{array}{l}\text { seen charged plus neutral particles } \\
\text { corrected to final } \\
\text { charged plus neutral particles }\end{array}$ \\
\hline & \pm 0.12 & 0.64 & \pm 0.64 \\
\hline $0.010-0.020$ & $12.230 \pm 0.054 \pm$ & 0.122 & $11.589 \pm 0.052 \pm 0.116$ \\
\hline $0.020-0.030$ & $6.128 \pm 0.038 \pm$ & 0.061 & $6.040 \pm 0.038 \pm 0.060$ \\
\hline $0.030-0.040$ & $3.828 \pm 0.030 \pm$ & 0.038 & $3.823 \pm 0.030 \pm$ \\
\hline $0.040-0.050$ & $2.756 \pm 0.025 \pm$ & 0.030 & $2.697 \pm 0.026 \pm 0.027$ \\
\hline $0.050-0.060$ & $1.995 \pm 0.022 \pm$ & 0.028 & $2.041 \pm 0.022 \pm 0.020$ \\
\hline $0.060-0.080$ & $1.470 \pm 0.013 \pm$ & 0.025 & $1.410 \pm 0.013 \pm 0.014$ \\
\hline $0.080-0.100$ & $0.932 \pm 0.011 \pm$ & 0.019 & $0.952 \pm 0.011 \pm 0.011$ \\
\hline $0.100-0.120$ & $0.6551 \pm 0.0091 \pm$ & 0.0162 & $0.6708 \pm 0.0090 \pm 0.0102$ \\
\hline $0.120-0.140$ & $0.4985 \pm 0.0081 \pm$ & 0.0144 & $0.4831 \pm 0.0077 \pm 0.0098$ \\
\hline $0.140-0.160$ & $0.3442 \pm 0.0065 \pm$ & 0.0114 & $0.3660 \pm 0.0068 \pm 0.0098$ \\
\hline $0.160-0.180$ & $0.2619 \pm 0.0059 \pm$ & 0.0103 & $0.2830 \pm 0.0061 \pm 0.0102$ \\
\hline $0.180-0.200$ & $0.2023 \pm 0.0055 \pm$ & 0.0099 & $0.2190 \pm 0.0054 \pm 0.0109$ \\
\hline $0.200-0.250$ & $0.0978 \pm 0.0024 \pm$ & 0.0060 & $0.1357 \pm 0.0028 \pm 0.0092$ \\
\hline $0.250-0.300$ & $0.0248 \pm 0.0013 \pm$ & 0.0019 & $0.0605 \pm 0.0022 \pm 0.0055$ \\
\hline
\end{tabular}

Table 37: Differential 2-jet rate for the Durham Algorithm, $D_{2}^{D}$

\begin{tabular}{|c|c|c|}
\hline Diff. Jet Ra & $D_{2}$ Jade Al & \\
\hline Interval & $\begin{array}{l}\text { seen charged particles } \\
\text { corrected to final } \\
\text { charged particles }\end{array}$ & $\begin{array}{l}\text { seen charged plus neutral particles } \\
\text { corrected to final } \\
\text { charged plus neutral particles }\end{array}$ \\
\hline $0.000-0.010$ & $26.014 \pm 0.077 \pm 0.260$ & $26.317 \pm 0.078 \pm 0.289$ \\
\hline $0.010-0.020$ & $18.243 \pm 0.065 \pm 0.182$ & $17.670 \pm 0.064 \pm 0.197$ \\
\hline $0.020-0.030$ & $11.945 \pm 0.054 \pm 0.119$ & $11.622 \pm 0.054 \pm 0.134$ \\
\hline $0.030-0.040$ & $8.378 \pm 0.046 \pm 0.084$ & $8.177 \pm 0.045 \pm 0.099$ \\
\hline $0.040-0.050$ & $6.218 \pm 0.039 \pm 0.062$ & $6.053 \pm 0.039 \pm 0.078$ \\
\hline $0.050-0.060$ & $4.817 \pm 0.035 \pm 0.048$ & $4.731 \pm 0.035 \pm 0.065$ \\
\hline $0.060-0.080$ & $3.490 \pm 0.021 \pm 0.035$ & $3.479 \pm 0.021 \pm 0.051$ \\
\hline $0.080-0.100$ & $2.358 \pm 0.017 \pm 0.024$ & $2.434 \pm 0.018 \pm 0.038$ \\
\hline $0.100-0.120$ & $1.680 \pm 0.014 \pm 0.020$ & $1.740 \pm 0.015 \pm 0.029$ \\
\hline $0.120-0.140$ & $1.247 \pm 0.012 \pm 0.017$ & $1.314 \pm 0.013 \pm 0.023$ \\
\hline $0.140-0.160$ & $0.941 \pm 0.011 \pm 0.016$ & $0.981 \pm 0.011 \pm 0.018$ \\
\hline $0.160-0.180$ & $0.7392 \pm 0.0097 \pm 0.0154$ & $0.7495 \pm 0.0098 \pm 0.0150$ \\
\hline $0.180-0.200$ & $0.5596 \pm 0.0084 \pm 0.0153$ & $0.5711 \pm 0.0086 \pm 0.0126$ \\
\hline $0.200-0.250$ & $0.3330 \pm 0.0042 \pm 0.0120$ & $0.3633 \pm 0.0044 \pm 0.0091$ \\
\hline $0.250-0.300$ & $0.1317 \pm 0.0028 \pm 0.0062$ & $0.1621 \pm 0.0030 \pm 0.0046$ \\
\hline
\end{tabular}

Table 38: Differential 2-jet rate for the Jade Algorithm, $D_{2}^{J}$ 


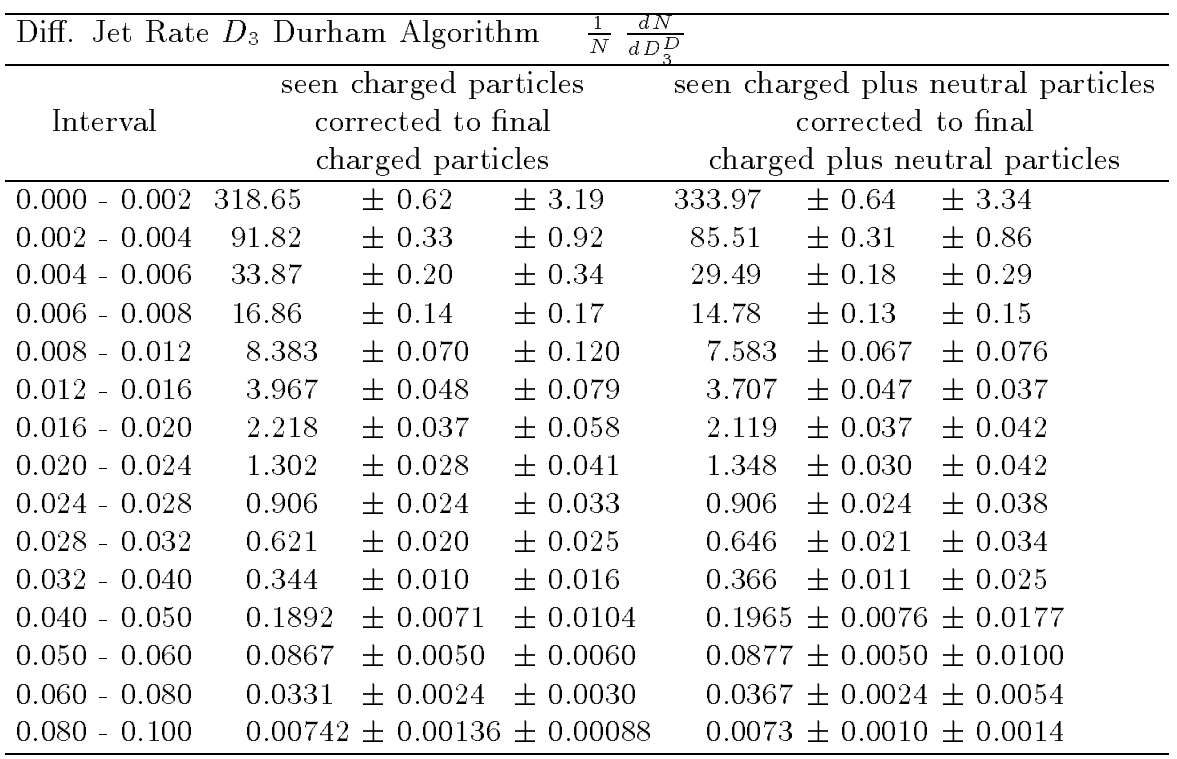

Table 39: Differential 3-jet rate for the Durham Algorithm, $D_{3}^{D}$

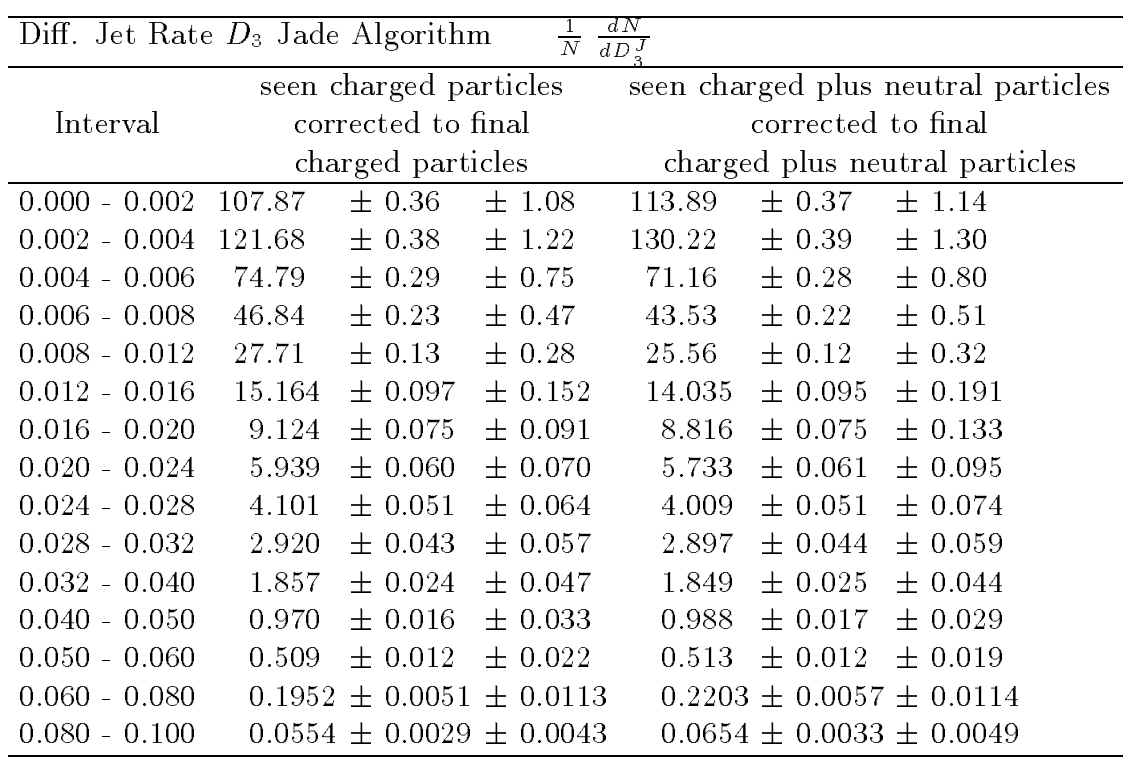

Table 40: Differential 3-jet rate for the Jade Algorithm, $D_{3}^{J}$ 


\begin{tabular}{|c|c|c|c|c|c|c|}
\hline \multirow{2}{*}{$\begin{array}{c}\text { Interval } \\
0.000-0.001\end{array}$} & \multicolumn{3}{|c|}{$\begin{array}{l}\text { seen charged particles } \\
\text { corrected to final } \\
\text { charged particles }\end{array}$} & \multicolumn{3}{|c|}{$\begin{array}{l}\text { seen charged plus neutral particles } \\
\text { corrected to final } \\
\text { charged plus neutral particles }\end{array}$} \\
\hline & 688.3 & \pm 1.3 & \pm 6.9 & 711.0 & \pm 1.3 & \pm 7.1 \\
\hline $0.001-0.002$ & 190.40 & \pm 0.67 & \pm 1.90 & 184.32 & \pm 0.66 & \pm 1.84 \\
\hline $0.002-0.003$ & 60.98 & \pm 0.37 & \pm 0.61 & 53.35 & \pm 0.35 & \pm 1.06 \\
\hline $0.003-0.004$ & 25.69 & \pm 0.24 & \pm 0.26 & 21.18 & \pm 0.22 & \pm 0.53 \\
\hline $0.004-0.005$ & 12.63 & \pm 0.17 & \pm 0.18 & 10.15 & \pm 0.15 & \pm 0.30 \\
\hline $0.005-0.006$ & 6.89 & \pm 0.13 & \pm 0.15 & 5.67 & \pm 0.11 & \pm 0.20 \\
\hline $0.006-0.008$ & 3.442 & \pm 0.063 & \pm 0.112 & 2.768 & \pm 0.057 & \pm 0.114 \\
\hline $0.008-0.010$ & 1.454 & \pm 0.040 & \pm 0.068 & 1.203 & \pm 0.039 & \pm 0.060 \\
\hline $0.010-0.012$ & 0.750 & \pm 0.031 & \pm 0.045 & 0.617 & \pm 0.028 & \pm 0.036 \\
\hline $0.012-0.014$ & 0.348 & \pm 0.020 & \pm 0.025 & 0.357 & \pm 0.022 & \pm 0.024 \\
\hline $0.014-0.016$ & 0.204 & \pm 0.016 & \pm 0.017 & 0.215 & \pm 0.017 & \pm 0.016 \\
\hline $0.016-0.018$ & 0.130 & \pm 0.013 & \pm 0.013 & 0.144 & \pm 0.015 & \pm 0.011 \\
\hline $0.018-0.020$ & 0.0712 & \pm 0.0092 & \pm 0.0078 & 0.0745 & \pm 0.010 & \pm 0.0064 \\
\hline $0.020-0.025$ & 0.0342 & \pm 0.0041 & \pm 0.0044 & 0.0434 & \pm 0.005 & \pm 0.0042 \\
\hline $0.025-0.030$ & 0.0110 & \pm 0.0023 & \pm 0.0017 & 0.0133 & \pm 0.0028 & \pm 0.0014 \\
\hline
\end{tabular}

Table 41: Differential 4-jet rate for the Durham Algorithm, $D_{4}^{D}$

\begin{tabular}{|c|c|c|c|c|}
\hline \multirow{2}{*}{$\frac{\text { Interval }}{0.000-0.001}$} & \multicolumn{3}{|c|}{$\begin{array}{l}\text { seen charged particles } \\
\text { corrected to final } \\
\text { charged particles }\end{array}$} & $\begin{array}{l}\text { seen charged plus neutral particles } \\
\text { corrected to final } \\
\text { charged plus neutral particles }\end{array}$ \\
\hline & 248.15 & \pm 0.77 & \pm 2.48 & $240.63 \pm 0.78 \pm 4.58$ \\
\hline $0.001-0.002$ & 286.76 & \pm 0.82 & \pm 2.87 & $309.26 \pm 0.86 \pm 4.96$ \\
\hline $0.002-0.003$ & 167.03 & \pm 0.63 & \pm 1.67 & $171.69 \pm 0.64 \pm 2.39$ \\
\hline $0.003-0.004$ & 97.00 & \pm 0.48 & \pm 1.04 & $92.65 \pm 0.47 \pm 1.18$ \\
\hline $0.004-0.005$ & 59.58 & \pm 0.38 & \pm 0.71 & $52.87 \pm 0.35 \pm 0.65$ \\
\hline $0.005-0.006$ & 37.48 & \pm 0.30 & \pm 0.49 & $33.41 \pm 0.28 \pm 0.43$ \\
\hline $0.006-0.008$ & 21.61 & \pm 0.16 & \pm 0.32 & $18.83 \pm 0.15 \pm 0.27$ \\
\hline $0.008-0.010$ & 11.10 & \pm 0.12 & \pm 0.19 & $9.45 \pm 0.11 \pm 0.17$ \\
\hline $0.010-0.012$ & 6.235 & \pm 0.087 & \pm 0.124 & $5.567 \pm 0.086 \pm 0.122$ \\
\hline $0.012-0.014$ & 3.602 & \pm 0.065 & \pm 0.080 & $3.262 \pm 0.066 \pm 0.089$ \\
\hline $0.014-0.016$ & 2.205 & \pm 0.052 & \pm 0.055 & $2.047 \pm 0.052 \pm 0.070$ \\
\hline $0.016-0.018$ & 1.373 & \pm 0.041 & \pm 0.037 & $1.330 \pm 0.042 \pm 0.055$ \\
\hline $0.018-0.020$ & 0.874 & \pm 0.032 & \pm 0.026 & $0.912 \pm 0.036 \pm 0.044$ \\
\hline $0.020-0.025$ & 0.460 & \pm 0.015 & \pm 0.016 & $0.456 \pm 0.016 \pm 0.025$ \\
\hline $0.025-0.030$ & 0.1735 & \pm 0.0096 & \pm 0.0070 & $0.195 \pm 0.011 \pm 0.012$ \\
\hline $0.030-0.050$ & 0.0303 & \pm 0.002 & \pm 0.0017 & $0.034 \pm 0.003 \pm 0.002$ \\
\hline
\end{tabular}

Table 42: Differential 4-jet rate for the Jade Algorithm, $D_{4}^{J}$ 


\begin{tabular}{|c|c|}
\hline \multirow{2}{*}{\multicolumn{2}{|c|}{$\begin{array}{cc}\text { Energy Energy Correlation } E E C \\
& \text { seen charged particles } \\
\text { Interval } & \text { corrected to final } \\
\cos \chi & \text { charged particles }\end{array}$}} \\
\hline & \\
\hline$-1.000--0.960$ & $3.0850 \pm 0.0085 \pm 0.0154$ \\
\hline $.960--0.9$ & $0.7097 \pm 0.0040 \pm 0$. \\
\hline $0--0.8$ & $0.3794 \pm 0.0029 \pm 0.0$ \\
\hline 0 & $0.2517 \pm 0.0024 \pm 0.0$ \\
\hline 0 & $0.18476 \pm 0.00202 \pm 0$. \\
\hline 50 & $0.14645 \pm 0.00179 \pm 0$ \\
\hline & $995 \pm 0$ \\
\hline & $55 \pm 0$ \\
\hline $0-0$ & $66 \pm 0$ \\
\hline & $132 \pm$ \\
\hline & $92 \pm$ \\
\hline & $98 \pm$ \\
\hline & 0.0 \\
\hline & $69 \pm$ \\
\hline 0 & $9 \pm$ \\
\hline & \\
\hline & \\
\hline U & 0.0 \\
\hline & $2 \pm$ \\
\hline 0 & $0 \pm$ \\
\hline & \\
\hline & \\
\hline-0 & 0.0 \\
\hline & 0 . \\
\hline & \\
\hline-0.0 & 0. \\
\hline 0 & \\
\hline-0 . & \\
\hline 0 & \\
\hline-0 & 0 . \\
\hline-0.2 & \pm \\
\hline-0. & \pm \\
\hline $0-0.3$ & $9 \pm$ \\
\hline-0.3 & 0. \\
\hline-0 & 0 . \\
\hline-0.4 & $3 \pm$ \\
\hline-0.4 & $6 \pm$ \\
\hline $0-0.5$ & $9 \pm$ \\
\hline-0.5 & 0. \\
\hline $0-0.6$ & $2 \pm$ \\
\hline-0.0 & $9 \pm$ \\
\hline-0.6 & $6 \pm$ \\
\hline $0-0.7$ & $3 \pm$ \\
\hline-0.7 & $2 \pm$ \\
\hline-0.8 & $3 \pm$ \\
\hline $0-0.0$ & 0.1 \\
\hline $40-0.880$ & $0196 \pm 0.00086$ \\
\hline $0-0.9$ & 0.2539 \\
\hline $0-0.9$ & $\pm 0.0033 \pm 0$ \\
\hline $0.960-1.000$ & $2.8965 \pm 0.0083 \pm 0.0166$ \\
\hline
\end{tabular}

\begin{tabular}{cc}
\hline Asymmetry of the Energy Energy Correlation \\
$A E E C$
\end{tabular}

Table 44: Asymmetry of the Energy Energy Correlation, $A E E C$

Table 43: Energy Energy Correlation, EEC 


\section{Tables of Sensitivities}

\begin{tabular}{|c|c|c|c|c|c|c|c|c|c|c|c|}
\hline \multirow[b]{3}{*}{ property } & \multicolumn{11}{|c|}{ model parameter } \\
\hline & \multirow[b]{2}{*}{$a$} & \multirow[b]{2}{*}{$b$} & \multirow[b]{2}{*}{$\sigma_{q}$} & \multirow[b]{2}{*}{$\Lambda_{Q C D}$} & \multirow[b]{2}{*}{$Q_{0}$} & \multirow[b]{2}{*}{$\gamma_{s}$} & \multirow[b]{2}{*}{$\frac{P(q q)}{P(q)}$} & \multicolumn{2}{|c|}{ u/d quarks } & \multicolumn{2}{|c|}{ s quarks } \\
\hline & & & & & & & & $P\left({ }^{1} S_{0}\right)$ & $P\left({ }^{3} S_{1}\right)$ & $P\left({ }^{1} S_{0}\right)$ & $P\left({ }^{3} S_{1}\right)$ \\
\hline $1-T$ & 3 & 10 & 9 & 48 & 1 & 4 & 2 & 1 & 1 & 1 & 1 \\
\hline$M$ & 11 & 21 & 21 & 81 & 9 & 3 & 4 & 2 & 3 & 2 & 2 \\
\hline$m$ & 11 & 27 & 20 & 77 & 9 & 7 & 2 & 3 & 1 & 3 & 1 \\
\hline$O$ & 4 & 8 & 7 & 26 & 2 & 3 & 3 & 1 & 1 & 1 & 1 \\
\hline$S$ & 2 & 5 & 8 & 47 & 3 & 4 & 2 & 1 & 1 & 1 & 1 \\
\hline$A$ & 3 & 17 & 22 & 80 & 4 & 9 & 5 & 3 & 3 & 4 & 1 \\
\hline$P$ & 2 & 4 & 5 & 39 & 1 & 3 & 4 & 1 & 1 & 2 & 1 \\
\hline$C$ & 3 & 10 & 10 & 51 & 2 & 5 & 3 & 1 & 1 & 1 & 1 \\
\hline$D$ & 5 & 15 & 14 & 77 & 4 & 5 & 2 & 1 & 3 & 2 & 1 \\
\hline$M_{h}^{2} / E_{v i s .}^{2}$ & 4 & 10 & 5 & 41 & 2 & 7 & 1 & 1 & 1 & 2 & 1 \\
\hline$M_{l}^{2} / E_{v i s .}^{2}$ & 7 & 17 & 1 & 47 & 4 & 7 & 1 & 1 & 1 & 0 & 0 \\
\hline$M_{d}^{2} / E_{\text {vis. }}^{2}$ & 2 & 6 & 4 & 27 & 2 & 4 & 4 & 1 & 1 & 3 & 1 \\
\hline$B_{\max }$ & 3 & 10 & 13 & 45 & 2 & 7 & 2 & 1 & 1 & 0 & 0 \\
\hline$B_{\min }$ & 6 & 15 & 14 & 56 & 2 & 1 & 2 & 1 & 0 & 1 & 1 \\
\hline$B_{\text {sum }}$ & 7 & 9 & 21 & 65 & 7 & 6 & 3 & 2 & 1 & 2 & 1 \\
\hline$B_{\text {diff }}$ & 2 & 3 & 8 & 27 & 1 & 8 & 1 & 1 & 1 & 1 & 1 \\
\hline$D_{2}^{D}$ & 2 & 2 & 9 & 33 & 2 & 6 & 2 & 1 & 1 & 1 & 1 \\
\hline$D_{3}^{D}$ & 3 & 9 & 16 & 87 & 4 & 11 & 7 & 3 & 5 & 4 & 2 \\
\hline$D_{4}^{D}$ & 7 & 21 & 36 & 113 & 9 & 18 & 8 & 2 & 7 & 3 & 4 \\
\hline$D_{2}^{J}$ & 0 & 3 & 5 & 39 & 2 & 4 & 3 & 1 & 1 & 1 & 1 \\
\hline$D_{3}^{J}$ & 4 & 10 & 14 & 71 & 1 & 6 & 2 & 1 & 2 & 1 & 2 \\
\hline$D_{4}^{J}$ & 6 & 25 & 21 & 90 & 4 & 15 & 8 & 2 & 2 & 2 & 2 \\
\hline$E E C$ & 2 & 6 & 4 & 34 & 1 & 3 & 1 & 1 & 0 & 1 & 0 \\
\hline$A E E C$ & 3 & 7 & 18 & 36 & 5 & 8 & 6 & 2 & 3 & 2 & 2 \\
\hline$p_{t}^{2 n}$ & 11 & 19 & 16 & 23 & 6 & 4 & 3 & 7 & 4 & 3 & 0 \\
\hline$p_{t}^{\text {out }}$ & 9 & 15 & 43 & 47 & 3 & 3 & 2 & 10 & 6 & 4 & 1 \\
\hline$y_{T}$ & 5 & 12 & 16 & 29 & 3 & 3 & 1 & 5 & 1 & 2 & 0 \\
\hline$x_{p}$ & 6 & 13 & 13 & 22 & 9 & 3 & 1 & 5 & 2 & 3 & 0 \\
\hline$\rho^{0}$ & 17 & 30 & 18 & 42 & 23 & 18 & 9 & 11 & 51 & 8 & 4 \\
\hline$K^{0} / K^{ \pm}$ & 10 & 21 & 15 & 41 & 13 & 57 & 4 & 2 & 6 & 25 & 6 \\
\hline$K^{* 0} / K^{* \pm}$ & 8 & 20 & 11 & 32 & 10 & 52 & 3 & 3 & 2 & 25 & 38 \\
\hline$\phi$ & 6 & 15 & 11 & 34 & 9 & 81 & 5 & 6 & 5 & 6 & 78 \\
\hline$p$ & 12 & 27 & 10 & 40 & 12 & 8 & 75 & 10 & 3 & 7 & 3 \\
\hline$\Lambda^{0}$ & 9 & 34 & 18 & 37 & 11 & 35 & 75 & 5 & 2 & 4 & 2 \\
\hline$\Sigma^{ \pm}$ & 8 & 23 & 15 & 40 & 15 & 34 & 73 & 4 & 6 & 9 & 7 \\
\hline
\end{tabular}

Table 45: Average sensitivities $(\times 100)$ for JETSET 7.4 PS with standard decays. Sensitivities for ARIADNE (with $p_{t}^{Q C D}$ in place of $Q_{0}$ ) and JETSET 7.3 PS are similar. The identified particle sensitivities have been calculated for the $\xi_{p}$ distributions. The quoted values have statistical errors. 


\begin{tabular}{|c|c|c|c|c|c|c|c|c|}
\hline \multirow[b]{2}{*}{ Property } & \multicolumn{8}{|c|}{ model parameter } \\
\hline & $Q C D L A M$ & RMASS(13) & $C L M A X$ & $C L P O W$ & $C L S M R$ & $P W T(3)$ & $P W T(7)$ & $D E C W T$ \\
\hline $1-T$ & 25 & 14 & 3 & 2 & 1 & 1 & 2 & 3 \\
\hline$M$ & 32 & 15 & 5 & 12 & 3 & 1 & 2 & 3 \\
\hline$m$ & 34 & 11 & 8 & 8 & 3 & 2 & 4 & 4 \\
\hline$O$ & 19 & 3 & 6 & 4 & 0 & 1 & 1 & 1 \\
\hline$S$ & 24 & 8 & 5 & 7 & 2 & 0 & 1 & 1 \\
\hline$A$ & 50 & 7 & 13 & 11 & 4 & 1 & 3 & 1 \\
\hline$P$ & 22 & 5 & 5 & 4 & 1 & 0 & 0 & 0 \\
\hline$C$ & 22 & 5 & 5 & 4 & 1 & 1 & 2 & 3 \\
\hline$D$ & 30 & 14 & 4 & 3 & 1 & 2 & 4 & 4 \\
\hline$M_{h}^{2} / E_{v i s .}^{2}$ & 20 & 13 & 8 & 5 & 1 & 1 & 1 & 1 \\
\hline$M_{l}^{2} / E_{v i s .}^{2}$ & 22 & 22 & 7 & 2 & 1 & 0 & 0 & 0 \\
\hline$M_{d}^{2} / E_{v i s .}^{2}$ & 18 & 6 & 8 & 6 & 1 & 1 & 1 & 1 \\
\hline$B_{\max }$ & 33 & 12 & 7 & 6 & 1 & 4 & 2 & 5 \\
\hline$B_{\min }$ & 40 & 14 & 6 & 5 & 2 & 2 & 2 & 2 \\
\hline$B_{\text {sum }}$ & 29 & 17 & 6 & 22 & 6 & 2 & 4 & 3 \\
\hline$B_{\text {diff }}$ & 20 & 3 & 4 & 3 & 1 & 1 & 1 & 2 \\
\hline$D_{2}^{D}$ & 27 & 4 & 4 & 2 & 1 & 2 & 1 & 1 \\
\hline$D_{3}^{D}$ & 53 & 9 & 8 & 4 & 2 & 1 & 2 & 1 \\
\hline$D_{4}^{D}$ & 74 & 11 & 17 & 12 & 4 & 4 & 8 & 2 \\
\hline$D_{2}^{J}$ & 14 & 4 & 2 & 2 & 1 & 1 & 1 & 1 \\
\hline$D_{3}^{J}$ & 38 & 9 & 7 & 7 & 1 & 1 & 2 & 3 \\
\hline$D_{4}^{J}$ & 44 & 12 & 15 & 10 & 2 & 1 & 4 & 3 \\
\hline$E E C$ & 18 & 6 & 2 & 2 & 1 & 0 & 1 & 1 \\
\hline$A E E C$ & 25 & 6 & 8 & 6 & 2 & 1 & 2 & 3 \\
\hline$p_{t}^{2 n}$ & 14 & 9 & 13 & 19 & 6 & 1 & 2 & 1 \\
\hline$p_{t}^{o u t}$ & 30 & 10 & 30 & 13 & 4 & 1 & 2 & 3 \\
\hline$y_{T}$ & 12 & 10 & 17 & 22 & 5 & 2 & 2 & 2 \\
\hline$x_{p}$ & 4 & 6 & 10 & 25 & 7 & 2 & 1 & 1 \\
\hline$\rho^{0}$ & 12 & 15 & 14 & 36 & 7 & 14 & 9 & 9 \\
\hline$K^{0} / K^{ \pm}$ & 16 & 28 & 39 & 55 & 12 & 32 & 7 & 7 \\
\hline$K^{* 0} / K^{* \pm}$ & 22 & 39 & 16 & 41 & 9 & 45 & 12 & 11 \\
\hline$\phi$ & 43 & 74 & 34 & 44 & 13 & 117 & 15 & 10 \\
\hline$p$ & 16 & 21 & 199 & 119 & 15 & 7 & 61 & 55 \\
\hline$\Lambda^{0}$ & 29 & 40 & 262 & 100 & 15 & 58 & 60 & 41 \\
\hline$\Sigma^{ \pm}$ & 44 & 58 & 187 & 64 & 35 & 42 & 60 & 17 \\
\hline
\end{tabular}

Table 46: Average sensitivities $(\times 100)$ for HERWIG $5.8 \mathrm{C}$ with standard decays. The identified particle sensitivities have been calculated for the $\xi_{p}$ distributions. The quoted values have statistical errors.

\begin{tabular}{|c|c|c|c|c|c|c|c|c|}
\hline \multirow[t]{2}{*}{ Parameter } & JETSET & $7.3 \mathrm{PS}$ & JETSET & $7.4 \mathrm{PS}$ & \multicolumn{2}{|c|}{ ARIADNE 4.06} & \multicolumn{2}{|c|}{ JETSET $7.4 \mathrm{ME}$} \\
\hline & $<x_{E}>_{D^{*}}$ & $<x_{E}>_{B}$ & $<x_{E}>_{D^{*}}$ & $<x_{E}>_{B}$ & $<x_{E}>_{D^{*}}$ & $<x_{E}>_{B}$ & $<x_{E}>_{D^{*}}$ & $<x_{E}>_{B}$ \\
\hline$\Lambda_{Q C D}$ & 32 & 30 & 29 & 26 & 23 & 18 & 22 & 23 \\
\hline$\epsilon_{c}$ & 9 & & 9 & & 10 & & 9 & \\
\hline$\epsilon_{b}$ & & 8 & & 8 & & 9 & & 10 \\
\hline
\end{tabular}

Table 47: Sensitivity $(\times 100)$ of $\left\langle x_{E}>\right.$ for $D^{*}$ and $B$ mesons 


\section{E Tables of the Results of the Fits}

\begin{tabular}{|c|c|c|c|c|c|c|}
\hline \multirow[t]{2}{*}{ Parameter } & \multirow[t]{2}{*}{ Name } & \multirow[t]{2}{*}{ Default } & \multirow[t]{2}{*}{ Range gen. } & \multicolumn{3}{|c|}{ Fit Result } \\
\hline & & & & Value & stat. & sys. \\
\hline$\Lambda_{Q C D}$ & $\operatorname{PARJ}(81)$ & 0.4 & $0.25-0.35$ & 0.297 & \pm 0.005 & $\begin{array}{l}+0.012 \\
-0.007\end{array}$ \\
\hline$Q_{0}$ & $\operatorname{PARJ}(82)$ & 1.0 & $1.0-2.0$ & 1.34 & \pm 0.08 & $\begin{array}{l}+\quad 0.15 \\
-0.09 \\
\end{array}$ \\
\hline$a$ & PARJ(41) & 0.5 & $0.1-0.5$ & 0.409 & \pm 0.020 & $\begin{array}{l}+0.009 \\
-0.013\end{array}$ \\
\hline$b$ & PARJ(42) & 0.9 & 0.844 & & optimized & \\
\hline$\sigma_{q}$ & PARJ(21) & 0.35 & $0.36-0.44$ & 0.401 & \pm 0.006 & $\begin{array}{l}+0.003 \\
-0.013 \\
\end{array}$ \\
\hline$P\left({ }^{1} S_{0}\right)_{u d}$ & - & 0.5 & $0.3-0.5$ & 0.272 & \pm 0.030 & $\begin{array}{l}+0.108 \\
-0.008\end{array}$ \\
\hline$P\left({ }^{3} S_{1}\right)_{u d}$ & - & 0.5 & $0.2-0.4$ & 0.306 & \pm 0.049 & $\begin{array}{l}+0.006 \\
-0.031\end{array}$ \\
\hline$P\left({ }^{1} P_{1}\right)_{u d}$ & - & 0 . & see text & & 0.094 & \\
\hline$P(\text { other } P \text { states })_{u d}$ & - & 0. & see text & & 0.305 & \\
\hline$\gamma_{s}$ & PARJ(2) & 0.30 & $0.27-0.31$ & 0.307 & \pm 0.007 & $\begin{array}{l}+0.002 \\
-0.017\end{array}$ \\
\hline$P\left({ }^{1} S_{0}\right)_{s}$ & - & 0.4 & $0.3-0.5$ & 0.416 & \pm 0.029 & $\begin{array}{l}+0.015 \\
-0.003\end{array}$ \\
\hline$P\left({ }^{3} S_{1}\right)_{s}$ & - & 0.6 & $0.2-0.4$ & 0.304 & \pm 0.023 & $\begin{array}{l}+0.017 \\
-0.002\end{array}$ \\
\hline$P(P \text { states })_{s}$ & - & 0. & see text & & 0.280 & \\
\hline$\epsilon_{c}$ & PARJ(54) & - & variable & -0.0372 & \pm 0.0008 & $\begin{array}{l}+0.0020 \\
-0.0012\end{array}$ \\
\hline$P\left({ }^{1} S_{0}\right)_{c}$ & - & 0.25 & 0.26 & & & \\
\hline$P\left({ }^{3} S_{1}\right)_{c}$ & - & 0.75 & 0.44 & & adj. to dat & \\
\hline$P(P \text { states })_{c}$ & - & 0. & 0.3 & & & \\
\hline$\epsilon_{b}$ & PARJ(55) & - & variable & -0.00284 & $4 \pm 0.00006$ & $\begin{array}{l}+0.00013 \\
-0.00009\end{array}$ \\
\hline$P\left({ }^{1} S_{0}\right)_{b}$ & - & 0.25 & 0.175 & & & \\
\hline$P\left({ }^{3} S_{1}\right)_{b}$ & - & 0.75 & 0.525 & & adj. to dat & \\
\hline$P(P \text { states })_{b}$ & - & 0. & 0.3 & & & \\
\hline$P(q q) / P(q)$ & PARJ(1) & 0.1 & $0.08-0.11$ & 0.095 & \pm 0.001 & $\begin{array}{l}+0.004 \\
-0.002\end{array}$ \\
\hline$[P(u s) / P(u d)] / \gamma_{s}$ & $\operatorname{PARJ}(3)$ & 0.4 & 0.593 & & adj. to dat & \\
\hline$P(q q 1) / P(q q 0)$ & $\operatorname{PARJ}(4)$ & 0.05 & 0.07 & & adj. to dat & \\
\hline extra baryon supp. & PARJ(19) & 0. & 0.5 & adj. to & o data, only & $y$ for uds \\
\hline extra $\eta$ supp. & PARJ(25) & 1.0 & 0.65 & & $0.65 \pm 0.0$ & \\
\hline extra $\eta^{\prime}$ supp. & PARJ(26) & 1.0 & 0.23 & & $0.23 \pm 0.0$ & \\
\hline
\end{tabular}

Table 48: Parameter settings and fit results for JETSET 7.3 PS with DELPHI decays 


\begin{tabular}{|c|c|c|c|c|c|c|}
\hline \multirow[t]{2}{*}{ Parameter } & \multirow[t]{2}{*}{ Name } & \multirow[t]{2}{*}{ Default } & \multirow[t]{2}{*}{ Range gen. } & \multicolumn{3}{|c|}{ Fit Result } \\
\hline & & & & Value & stat. & sys. \\
\hline$\Lambda_{Q C D}$ & PARJ(81) & 0.29 & $0.25-0.35$ & 0.297 & \pm 0.004 & $\begin{array}{l}+0.007 \\
-0.008\end{array}$ \\
\hline$Q_{0}$ & PARJ $(82)$ & 1.0 & $1.0-2.0$ & 1.56 & \pm 0.11 & $\begin{array}{l}+\quad 0.21 \\
-\quad 0.15 \\
\end{array}$ \\
\hline$a$ & $\operatorname{PARJ}(41)$ & 0.3 & $0.1-0.5$ & 0.417 & \pm 0.022 & $\begin{array}{l}+0.011 \\
-0.015\end{array}$ \\
\hline$b$ & PARJ(42) & 0.58 & 0.850 & & optimized & \\
\hline$\sigma_{q}$ & PARJ(21) & 0.36 & $0.36-0.44$ & 0.408 & \pm 0.005 & $\begin{array}{l}+0.004 \\
-0.004\end{array}$ \\
\hline$P\left({ }^{1} S_{0}\right)_{u d}$ & - & 0.5 & $0.3-0.5$ & 0.297 & \pm 0.021 & $\begin{array}{l}+0.102 \\
-0.011\end{array}$ \\
\hline$P\left({ }^{3} S_{1}\right)_{u d}$ & - & 0.5 & $0.2-0.4$ & 0.289 & \pm 0.038 & $\begin{array}{l}+0.004 \\
-0.026\end{array}$ \\
\hline$P\left({ }^{1} P_{1}\right)_{u d}$ & - & 0 . & see text & & 0.096 & \\
\hline$P(\text { other } P \text { states })_{u d}$ & - & 0. & see text & & 0.318 & \\
\hline$\gamma_{s}$ & $\operatorname{PARJ}(2)$ & 0.30 & $0.27-0.31$ & 0.308 & \pm 0.007 & $\begin{array}{l}+0.004 \\
-0.036\end{array}$ \\
\hline$P\left({ }^{1} S_{0}\right)_{s}$ & - & 0.4 & $0.3-0.5$ & 0.410 & \pm 0.038 & $\begin{array}{l}+0.026 \\
-0.013\end{array}$ \\
\hline$P\left({ }^{3} S_{1}\right)_{s}$ & - & 0.6 & $0.2-0.4$ & 0.297 & \pm 0.021 & $\begin{array}{l}+0.020 \\
-0.004\end{array}$ \\
\hline$P(P \text { states })_{s}$ & - & 0. & see text & & 0.293 & \\
\hline$\epsilon_{c}$ & PARJ(54) & - & variable & -0.0372 & \pm 0.0007 & $\begin{array}{l}+0.0011 \\
-0.0012\end{array}$ \\
\hline$P\left({ }^{1} S_{0}\right)_{c}$ & - & 0.25 & 0.26 & & & \\
\hline$P\left({ }^{3} S_{1}\right)_{c}$ & - & 0.75 & 0.44 & & adj. to dat & \\
\hline$P(P \text { states })_{c}$ & - & 0. & 0.3 & & & \\
\hline$\epsilon_{b}$ & PARJ(55) & - & variable & -0.00284 & $4 \pm 0.00005$ & $\begin{array}{l}+0.00012 \\
-0.00010\end{array}$ \\
\hline$P\left({ }^{1} S_{0}\right)_{b}$ & - & 0.25 & 0.175 & & & \\
\hline$P\left({ }^{3} S_{1}\right)_{b}$ & - & 0.75 & 0.525 & & adj. to dat & \\
\hline$P(P \text { states })_{b}$ & - & 0. & 0.3 & & & \\
\hline$P(q q) / P(q)$ & PARJ(1) & 0.1 & $0.08-0.11$ & 0.099 & \pm 0.001 & $\begin{array}{l}+0.005 \\
-0.002\end{array}$ \\
\hline$[P(u s) / P(u d)] / \gamma_{s}$ & $\operatorname{PARJ}(3)$ & 0.4 & 0.593 & & adj. to dat & \\
\hline$P(q q 1) / P(q q 0)$ & $\operatorname{PARJ}(4)$ & 0.05 & 0.07 & & adj. to dat & \\
\hline extra baryon supp. & PARJ(19) & 0. & 0.5 & adj. to & o data, only & $y$ for uds \\
\hline extra $\eta$ supp. & PARJ(25) & 1.0 & 0.65 & & $0.65 \pm 0.0$ & \\
\hline extra $\eta^{\prime}$ supp. & PARJ(26) & 1.0 & 0.23 & & $0.23 \pm 0.0$ & \\
\hline
\end{tabular}

Table 49: Parameter settings and fit results for JETSET 7.4 PS with default decays 


\begin{tabular}{|c|c|c|c|c|c|c|}
\hline \multirow[t]{2}{*}{ Parameter } & \multirow[t]{2}{*}{ Name } & \multirow[t]{2}{*}{ Default } & \multirow[t]{2}{*}{ Range gen. } & \multicolumn{3}{|c|}{ Fit Result } \\
\hline & & & & Value & stat. & sys. \\
\hline$\Lambda_{Q C D}$ & $\operatorname{PARA}(1)$ & 0.22 & $0.20-0.30$ & 0.237 & \pm 0.003 & $\begin{array}{l}+0.006 \\
-0.002\end{array}$ \\
\hline$p_{t}^{Q C D}$ & $\operatorname{PARA}(3)$ & 0.6 & $0.4-1.2$ & 0.64 & \pm 0.08 & $\begin{array}{l}+0.20 \\
-0.08 \\
\end{array}$ \\
\hline$a$ & PARJ(41) & 0.5 & $0.1-0.5$ & 0.391 & \pm 0.047 & $\begin{array}{l}+0.039 \\
-0.046\end{array}$ \\
\hline$b$ & PARJ(42) & 0.9 & 0.850 & & optimized & \\
\hline$\sigma_{q}$ & PARJ(21) & 0.35 & $0.36-0.44$ & 0.390 & \pm 0.005 & $\begin{array}{l}+0.003 \\
-0.007\end{array}$ \\
\hline$P\left({ }^{1} S_{0}\right)_{u d}$ & - & 0.5 & $0.3-0.5$ & 0.276 & \pm 0.017 & $\begin{array}{l}+0.103 \\
-0.012\end{array}$ \\
\hline$P\left({ }^{3} S_{1}\right)_{u d}$ & - & 0.5 & $0.2-0.4$ & 0.292 & \pm 0.056 & $\begin{array}{l}+0.003 \\
-0.020\end{array}$ \\
\hline$P\left({ }^{1} P_{1}\right)_{u d}$ & - & 0. & see text & & 0.096 & \\
\hline$P(\text { other } P \text { states })_{u d}$ & - & 0. & see text & & 0.336 & \\
\hline$\gamma_{s}$ & PARJ(2) & 0.30 & $0.27-0.31$ & 0.302 & \pm 0.004 & $\begin{array}{l}+0.004 \\
-0.008\end{array}$ \\
\hline$P\left({ }^{1} S_{0}\right)_{s}$ & - & 0.4 & $0.3-0.5$ & 0.389 & \pm 0.026 & $\begin{array}{l}+0.012 \\
-0.010\end{array}$ \\
\hline$P\left({ }^{3} S_{1}\right)_{s}$ & - & 0.6 & $0.2-0.4$ & 0.301 & \pm 0.019 & $\begin{array}{l}+0.004 \\
-0.002\end{array}$ \\
\hline$P\left(P\right.$ states $_{s}$ & - & 0. & see text & & 0.310 & \\
\hline$\epsilon_{c}$ & PARJ(54) & - & variable & -0.0378 & \pm 0.0005 & $\begin{array}{l}+0.0009 \\
-0.0004\end{array}$ \\
\hline$P\left({ }^{1} S_{0}\right)_{c}$ & - & 0.25 & 0.26 & & & \\
\hline$P\left({ }^{3} S_{1}\right)_{c}$ & - & 0.75 & 0.44 & & adj. to dat & \\
\hline$P(P \text { states })_{c}$ & - & 0. & 0.3 & & & \\
\hline$\epsilon_{b}$ & PARJ(55) & - & variable & -0.00255 & $5 \pm 0.00004$ & $\begin{array}{l}+0.00006 \\
-0.00002\end{array}$ \\
\hline$P\left({ }^{1} S_{0}\right)_{b}$ & - & 0.25 & 0.175 & & & \\
\hline$P\left({ }^{3} S_{1}\right)_{b}$ & - & 0.75 & 0.525 & & adj. to dat & \\
\hline$P(P \text { states })_{b}$ & - & 0. & 0.3 & & & \\
\hline$P(q q) / P(q)$ & PARJ(1) & 0.1 & $0.08-0.11$ & 0.096 & \pm 0.001 & $\begin{array}{l}+0.008 \\
-0.001\end{array}$ \\
\hline$[P(u s) / P(u d)] / \gamma_{s}$ & $\operatorname{PARJ}(3)$ & 0.4 & 0.603 & & adj. to dat & \\
\hline$P(q q 1) / P(q q 0)$ & PARJ(4) & 0.05 & 0.07 & & adj. to dat & \\
\hline extra baryon supp. & PARJ(19) & 0. & 0.5 & adj. to & o data, only & $y$ for uds \\
\hline extra $\eta$ supp. & PARJ(25) & 1.0 & 0.65 & & $0.65 \pm 0.0$ & \\
\hline extra $\eta^{\prime}$ supp. & PARJ(26) & 1.0 & 0.23 & & $0.23 \pm 0.0$ & \\
\hline
\end{tabular}

Table 50: Parameter settings and fit results for ARIADNE 4.06 with DELPHI decays 


\begin{tabular}{|c|c|c|c|c|c|c|}
\hline \multirow[t]{2}{*}{ Parameter } & \multirow[t]{2}{*}{ name } & \multirow[t]{2}{*}{ Default } & \multirow[t]{2}{*}{ Range gen. } & \multicolumn{3}{|c|}{ Fit Result } \\
\hline & & & & Value & stat. & sys. \\
\hline$\Lambda_{Q C D}$ & PARA(1) & 0.22 & $0.20-0.30$ & 0.237 & $\pm 0.004 \quad$ & $\begin{array}{l}0.005 \\
-0.004\end{array}$ \\
\hline$p_{t}^{Q C D}$ & $\operatorname{PARA}(3)$ & 0.6 & $0.4-1.2$ & 0.68 & \pm 0.06 & $\begin{array}{l}0.11 \\
-0.11 \\
\end{array}$ \\
\hline$a$ & PAR.J(41) & 0.5 & $0.1-0.5$ & 0.388 & \pm 0.030 & $\begin{array}{r}-0.036 \\
-0.112\end{array}$ \\
\hline$b$ & PARJ(42) & 0.9 & 0.850 & & optimized & \\
\hline$\sigma_{q}$ & PARJ(21) & 0.35 & $0.36-0.44$ & 0.387 & $\pm 0.008 \quad$ & $\begin{array}{r}-0.012 \\
-0.016\end{array}$ \\
\hline$P\left({ }^{1} S_{0}\right)_{u d}$ & - & 0.5 & $0.3-0.5$ & 0.326 & \pm 0.017 & $\begin{array}{r}-0.058 \\
-0.023\end{array}$ \\
\hline$P\left({ }^{3} S_{1}\right)_{u d}$ & - & 0.5 & $0.2-0.4$ & 0.263 & $\pm 0.039 \quad+$ & $\begin{array}{r}0.015 \\
-0.010\end{array}$ \\
\hline$P\left({ }^{1} P_{1}\right)_{u d}$ & - & 0. & see text & & 0.096 & \\
\hline$P(\text { other } P \text { states })_{u d}$ & - & 0. & see text & & 0.411 & \\
\hline$\gamma_{s}$ & PARJ(2) & 0.30 & $0.27-0.31$ & 0.282 & $\pm 0.005 \quad+$ & $\begin{array}{r}-0.015 \\
-0.004\end{array}$ \\
\hline$P\left({ }^{1} S_{0}\right)_{s}$ & - & 0.4 & $0.3-0.5$ & 0.395 & \pm 0.023 & $\begin{array}{r}0.008 \\
-0.040\end{array}$ \\
\hline$P\left({ }^{3} S_{1}\right)_{s}$ & - & 0.6 & $0.2-0.4$ & 0.325 & $\pm 0.015 \quad+$ & $\begin{array}{r}0.005 \\
-0.033\end{array}$ \\
\hline$P(P \text { states })_{s}$ & - & 0. & see text & & 0.280 & \\
\hline$\epsilon_{c}$ & PARJ(54) & - & variable & -0.0378 & $\pm 0.0005 \stackrel{+}{-}$ & $\begin{array}{r}0.0009 \\
-0.0004\end{array}$ \\
\hline$P\left({ }^{1} S_{0}\right)_{c}$ & - & 0.25 & 0.26 & & & \\
\hline$P\left({ }^{3} S_{1}\right)_{c}$ & - & 0.75 & 0.44 & & adj. to data & \\
\hline$P(P \text { states })_{c}$ & - & 0. & 0.3 & & & \\
\hline$\epsilon_{b}$ & PAR.J(55) & - & variable & -0.00255 & $5 \pm 0.00004_{-}^{+}$ & $\begin{array}{r}0.00006 \\
-0.00002\end{array}$ \\
\hline$P\left({ }^{1} S_{0}\right)_{b}$ & - & 0.25 & 0.175 & & & \\
\hline$P\left({ }^{3} S_{1}\right)_{b}$ & - & 0.75 & 0.525 & & adj. to data & \\
\hline$P(P \text { states })_{b}$ & - & 0. & 0.3 & & & \\
\hline$P(q q) / P(q)$ & PARJ(1) & 0.1 & $0.08-0.11$ & 0.101 & \pm 0.001 & $\begin{array}{r}-0.006 \\
-0.001\end{array}$ \\
\hline$[P(u s) / P(u d)] / \gamma_{s}$ & $\operatorname{PARJ}(3)$ & 0.4 & 0.645 & & adj. to data & \\
\hline$P(q q 1) / P(q q 0)$ & $\operatorname{PARJ}(4)$ & 0.05 & 0.07 & & adj. to data & \\
\hline extra baryon supp. & PARJ(19) & 0. & 0.5 & adj. to & o data, only $f$ & for uds \\
\hline extra $\eta$ supp. & PARJ(25) & 1.0 & 0.65 & & $0.65 \pm 0.06$ & \\
\hline extra $\eta^{\prime}$ supp. & PARJ(26) & 1.0 & 0.23 & & $0.23 \pm 0.05$ & \\
\hline
\end{tabular}

Table 51: Parameter settings and fit results for ARIADNE 4.06 with default decays 


\begin{tabular}{|c|c|c|c|c|c|c|}
\hline \multirow[t]{2}{*}{ Parameter } & \multirow[t]{2}{*}{ Name } & \multirow[t]{2}{*}{ Default } & \multirow[t]{2}{*}{ Range gen. } & \multicolumn{3}{|c|}{ Fit Result } \\
\hline & & & & Value & stat. & sys. \\
\hline$\Lambda_{Q C D}$ & PARJ(122) & 0.22 & -0.30 & 0.163 & $\pm 0.002 \quad$ & $\begin{array}{r}+0.001 \\
-0.003\end{array}$ \\
\hline Scale $\mu$ & PARJ(129) & 0.002 & $0.0005-0.0025$ & 0.0025 & $0 \pm 0.00006_{-}^{+}$ & $\begin{array}{r}+0.00013 \\
-\quad 0.00018 \\
\end{array}$ \\
\hline$a$ & PARJ(41) & 0.3 & -0.5 & 0.903 & $\pm 0.014 \quad$ & $\begin{array}{r}0.038 \\
-0.014\end{array}$ \\
\hline$b$ & PARJ(42) & 0.58 & 0.525 & \multicolumn{3}{|c|}{ optimized } \\
\hline$\sigma_{q}$ & PARJ(21) & 0.36 & $0.36-0.44$ & 0.477 & 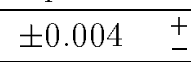 & $\begin{array}{r}+0.005 \\
-0.006 \\
\end{array}$ \\
\hline$P\left({ }^{1} S_{0}\right)_{u d}$ & - & 0.5 & -0.5 & 0.327 & \pm 0.023 & $\begin{array}{l}+0.051 \\
-0.021\end{array}$ \\
\hline$P\left({ }^{3} S_{1}\right)_{u d}$ & - & 0.5 & -0.4 & 0.274 & \pm 0.061 & $\begin{array}{r}0.012 \\
-0.013\end{array}$ \\
\hline$P\left({ }^{1} P_{1}\right)_{u d}$ & - & 0 . & see text & \multicolumn{3}{|c|}{0.089} \\
\hline$P(\text { other } P \text { states })_{u d}$ & - & 0. & see text & \multicolumn{3}{|c|}{0.310} \\
\hline$\gamma_{s}$ & $\operatorname{PARJ}(2)$ & 0.30 & $0.27-0.31$ & 0.277 & \pm 0.006 & $\begin{array}{l}+0.008 \\
-0.004\end{array}$ \\
\hline$P\left({ }^{1} S_{0}\right)_{s}$ & - & 0.4 & -0.5 & 0.413 & \pm 0.028 & $\begin{array}{l}0.016 \\
-0.011\end{array}$ \\
\hline$P\left({ }^{3} S_{1}\right)_{s}$ & - & 0.6 & -0.4 & 0.330 & $\pm 0.021 \quad$ & $\begin{array}{l}0.011 \\
-0.008\end{array}$ \\
\hline$P\left(P\right.$ states $_{s}$ & - & 0. & see text & \multicolumn{3}{|c|}{0.257} \\
\hline$\epsilon_{c}$ & PARJ(54) & - & -0.0950 & \multicolumn{3}{|c|}{ optimized } \\
\hline$P\left({ }^{1} S_{0}\right)_{c}$ & - & 0.25 & 0.26 & \\
\hline$P\left({ }^{3} S_{1}\right)_{c}$ & - & 0.75 & 0.44 & \multicolumn{3}{|c|}{ adj. to data } \\
\hline$P(P \text { states })_{c}$ & - & 0. & 0.3 & & & \\
\hline$\epsilon_{b}$ & PARJ $(55)$ & - & -0.00427 & \multicolumn{3}{|c|}{ optimized } \\
\hline$P\left({ }^{1} S_{0}\right)_{b}$ & - & 0.25 & 0.175 & \\
\hline$P\left({ }^{3} S_{1}\right)_{b}$ & - & 0.75 & 0.525 & \multicolumn{3}{|c|}{ adj. to data } \\
\hline$P(P \text { states })_{b}$ & - & 0. & 0.3 & & & \\
\hline$P(q q) / P(q)$ & PARJ(1) & 0.1 & $0.08-0.11$ & 0.087 & \pm 0.002 & $\begin{array}{r}+0.008 \\
-0.002\end{array}$ \\
\hline$[P(u s) / P(u d)] / \gamma_{s}$ & $\operatorname{PARJ}(3)$ & 0.4 & 0.657 & \multicolumn{3}{|c|}{ adj. to data } \\
\hline$P(q q 1) / P(q q 0)$ & PARJ(4) & 0.05 & 0.07 & \multicolumn{3}{|c|}{ adj. to data } \\
\hline extra baryon supp. & PARJ(19) & 0. & 0.5 & \multicolumn{3}{|c|}{ adj. to data, only for uds } \\
\hline \multirow{2}{*}{$\begin{array}{l}\text { extra } \eta \text { supp. } \\
\text { extra } \eta^{\prime} \text { supp. }\end{array}$} & PARJ $(25)$ & 1.0 & 0.65 & & $0.65 \pm 0.06$ & \\
\hline & PARJ(26) & 1.0 & 0.23 & & $0.23 \pm 0.05$ & \\
\hline
\end{tabular}

Table 52: Parameter settings and fit results for JETSET 7.4 ME with default decays

\begin{tabular}{llllll}
\hline Parameter & Default & Range gen. & \multicolumn{2}{c}{ Fit Result } \\
& & \multicolumn{4}{c}{ Value } \\
stat. & sys. \\
\hline QCDLAM & 0.18 & $0.155-0.205$ & $0.163 \pm 0.001 \pm 0.004$ \\
RMASS(13) & 0.75 & $0.64-0.96$ & $0.65 \pm 0.01 \pm 0.02$ \\
CLMAX & 3.35 & 2.35 & -4.35 & $3.48 \pm 0.04 \pm 0.01$ \\
CLPOW & 2.0 & 1.0 & -2.0 & $1.49 \pm 0.04 \pm 0.13$ \\
CLSMR & 0. & 0.0 & -1.0 & $0.36 \pm 0.04 \pm 0.06$ \\
DECWT & 1.0 & 0.0 & -1.0 & $0.77 \pm 0.08 \pm 0.01$ \\
PWT(3) & 1.0 & 0.0 & -1.0 & $0.83 \pm 0.02 \pm 0.03$ \\
PWT(7) & 1.0 & 0.0 & -1.0 & $0.74 \pm 0.09 \pm 0.09$ \\
\hline
\end{tabular}

Table 53: Parameter settings and fit results for HERWIG 5.8 C 


\begin{tabular}{|c|c|c|c|c|c|c|c|c|c|}
\hline & $a$ & $\sigma_{q}$ & $\Lambda_{Q C D}$ & $Q_{0}$ & $\gamma_{s}$ & $P\left({ }^{1} S_{0}\right)_{u d}$ & $P\left({ }^{3} S_{1}\right)_{u d}$ & $P\left({ }^{1} S_{0}\right)_{s}$ & $P\left({ }^{3} S_{1}\right)_{s}$ \\
\hline$a$ & 1.00 & 0.33 & -0.60 & 0.01 & -0.10 & 0.27 & -0.11 & 0.06 & 0.03 \\
\hline$\sigma_{q}$ & & 1.00 & -0.69 & -0.08 & 0.43 & -0.41 & 0.11 & -0.26 & -0.29 \\
\hline$\Lambda_{Q C D}$ & & & 1.00 & 0.18 & -0.26 & 0.19 & -0.10 & 0.15 & 0.20 \\
\hline$Q_{0}$ & & & & 1.00 & 0.20 & 0.06 & -0.38 & -0.35 & -0.13 \\
\hline$\gamma_{s}$ & & & & & 1.00 & -0.04 & 0.19 & -0.73 & -0.65 \\
\hline$P\left({ }^{1} S_{0}\right)_{u d}$ & & & & & & 1.00 & 0.37 & -0.29 & 0.12 \\
\hline$P\left({ }^{3} S_{1}\right)_{u d}$ & & & & & & & 1.00 & -0.10 & -0.26 \\
\hline$P\left({ }^{1} S_{0}\right)_{s}$ & & & & & & & & 1.00 & 0.41 \\
\hline$P\left({ }^{3} S_{1}\right)_{s}$ & & & & & & & & & 1.00 \\
\hline
\end{tabular}

Table 54: Table of Correlation Coefficients for JETSET 7.4 PS Fit

\begin{tabular}{|c|c|c|c|c|c|c|c|c|c|}
\hline & $a$ & $\sigma_{q}$ & $\Lambda_{Q C D}$ & $p_{t}^{Q C D}$ & $\gamma_{s}$ & $P\left({ }^{1} S_{0}\right)_{u d}$ & $P\left({ }^{3} S_{1}\right)_{u d}$ & $P\left({ }^{1} S_{0}\right)_{s}$ & $P\left({ }^{3} S_{1}\right)_{s}$ \\
\hline$a$ & 1.00 & 0.11 & -0.18 & 0.90 & -0.29 & 0.71 & -0.04 & 0.29 & 0.07 \\
\hline$\sigma_{q}$ & & 1.00 & -0.60 & -0.07 & 0.12 & -0.47 & -0.06 & 0.02 & 0.05 \\
\hline$\Lambda_{Q C D}$ & & & 1.00 & 0.16 & 0.02 & 0.18 & 0.03 & -0.03 & -0.06 \\
\hline$p_{t}^{Q C D}$ & & & & 1.00 & -0.21 & 0.65 & -0.02 & 0.26 & 0.05 \\
\hline$\gamma_{s}$ & & & & & 1.00 & -0.27 & -0.02 & -0.41 & -0.47 \\
\hline$P\left({ }^{1} S_{0}\right)_{u d}$ & & & & & & 1.00 & -0.11 & -0.03 & 0.13 \\
\hline$P\left({ }^{3} S_{1}\right)_{u d}$ & & & & & & & 1.00 & 0.08 & -0.03 \\
\hline$P\left({ }^{1} S_{0}\right)_{s}$ & & & & & & & & 1.00 & 0.02 \\
\hline$P\left({ }^{3} S_{1}\right)_{s}$ & & & & & & & & & 1.00 \\
\hline
\end{tabular}

Table 55: Table of Correlation Coefficients for ARIADNE 4.06 PS Fit 


\section{F Figures of Data and Model Comparisons}

The figures in this section compare corrected DELPHI data and identified particle spectra to the following fragmentation models:

- JETSET 7.3 with DELPHI decays labeled JT 7.3 PS (full curves)

- JETSET 7.4 with default decays labeled JT 7.4 PS (dashed curves)

- ARIADNE 4.06 with DELPHI decays labeled AR 4.06 (dotted curves)

- HERWIG 5.8 C with default decays labeled H $5.8 \mathrm{C}$ (dot-dashed curves)

- JETSET 7.4 ME with default decays labeled JT 7.4 ME (widely spaced dots)

The references for the identified particle data can be found in table 7 . The total correction factor for each distribution is shown in the upper inset. The lower insets of the plots show the relative deviation of the models from the data. Also shown as a shaded area in these insets is the total experimental error obtained by adding quadratically the systematic and statistical error in each bin. The error should be interpreted like a statistical one standard deviation uncertainty.

For the $\xi_{p}$ distributions of identified $K^{0}, K^{ \pm}, p$ and $\Lambda^{0}$ (Figs. 39, 40, 45, 46), we also show separate plots comparing the models with each data set separately (ALEPH dashed curve, DELPHI full curve, OPAL dotted curve). 


\section{F.1 Inclusive Single Charged Particle Distributions}

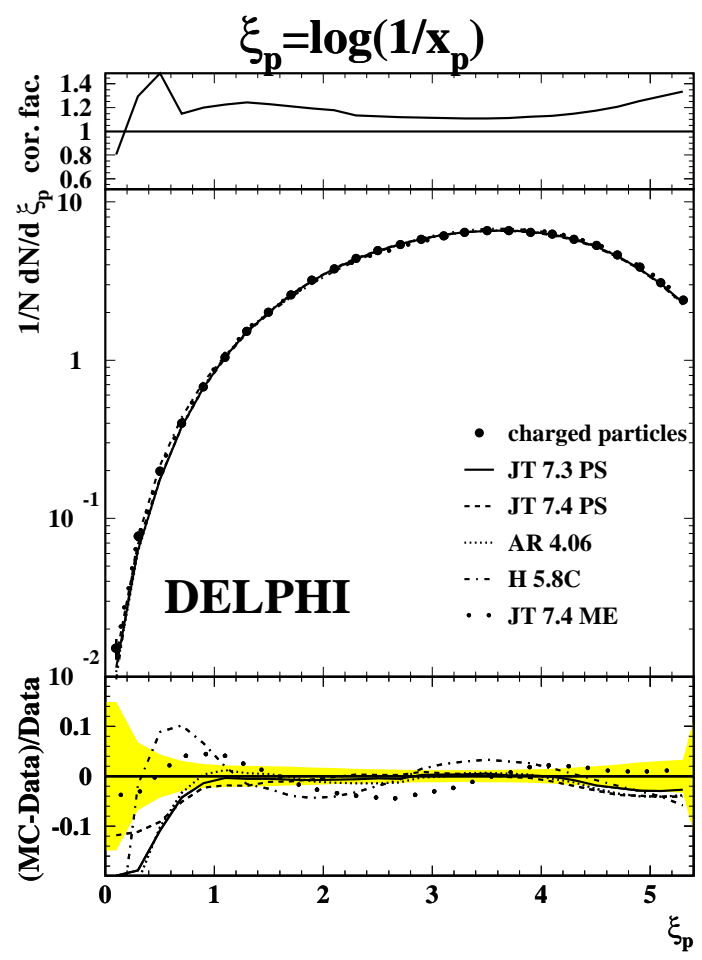

Figure $3: \xi_{p}=\log \left(1 / x_{p}\right)$

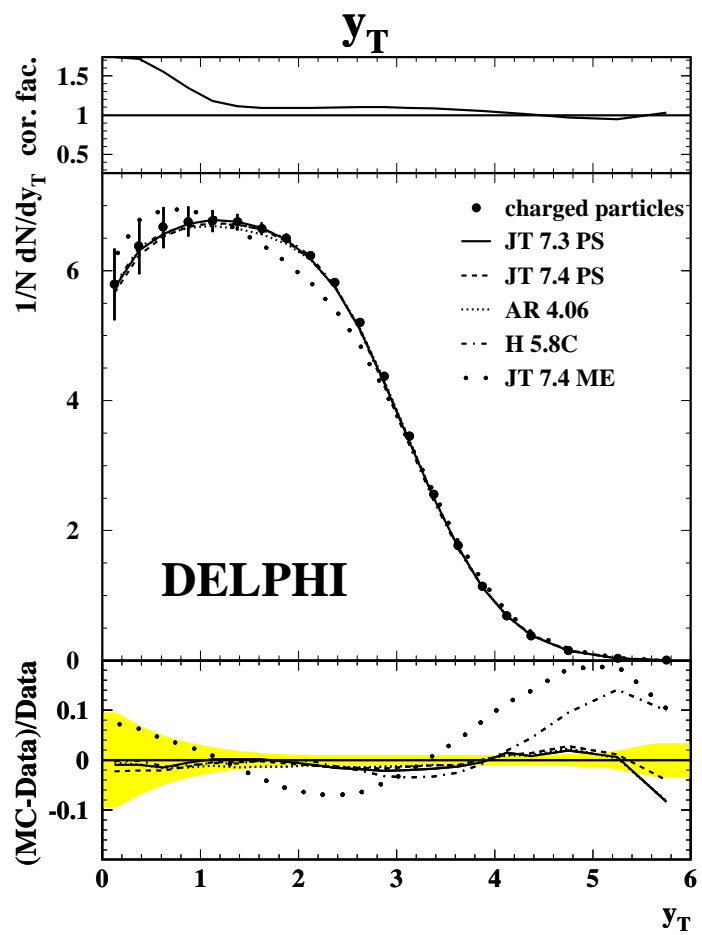

Figure 5: Rapidity, $y_{T}$

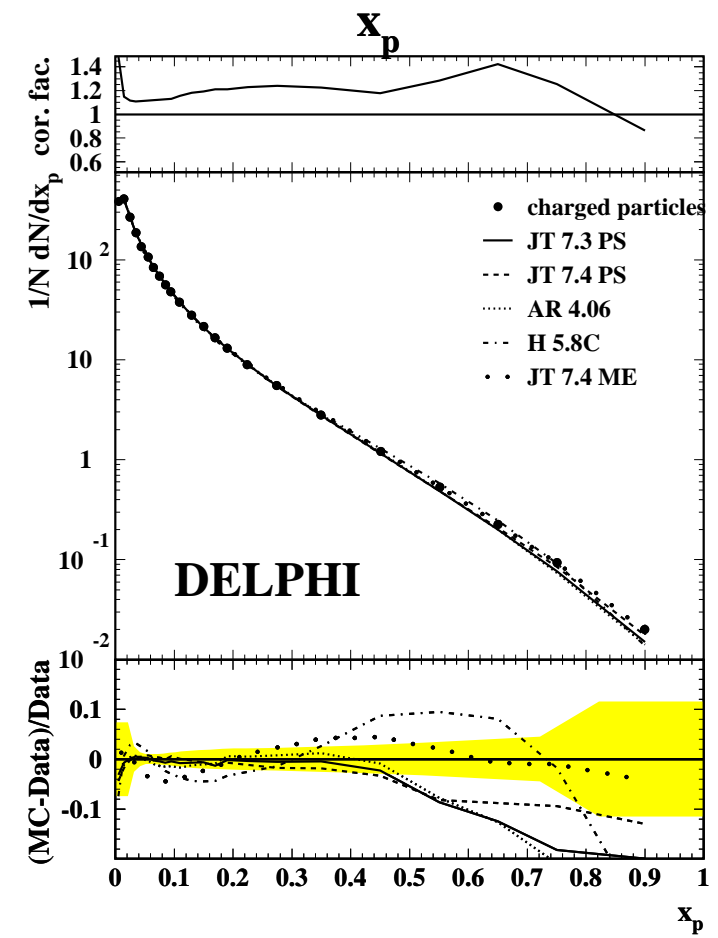

Figure 4: Scaled Momentum, $x_{p}$

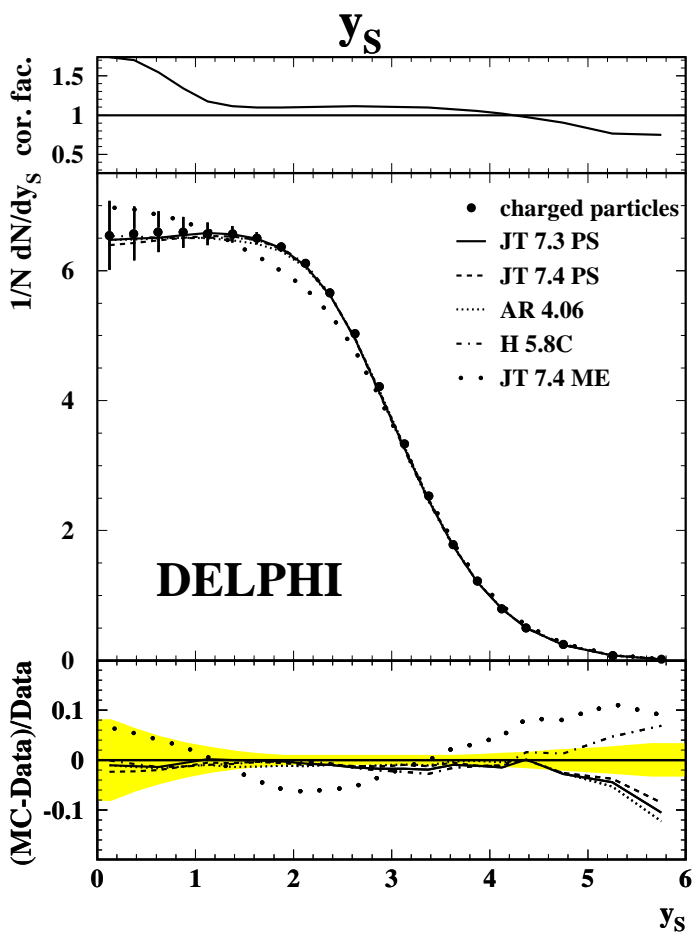

Figure 6: Rapidity, $y_{S}$ 


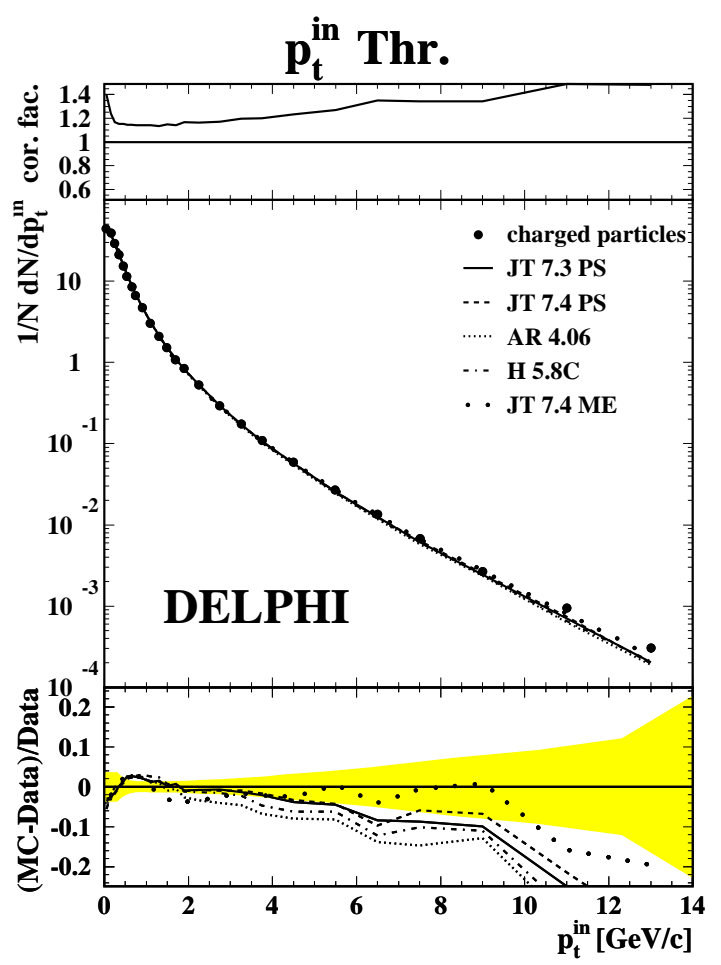

Figure 7: Transverse momentum, $p_{t}^{i n}$, with respect to the Thrust axis

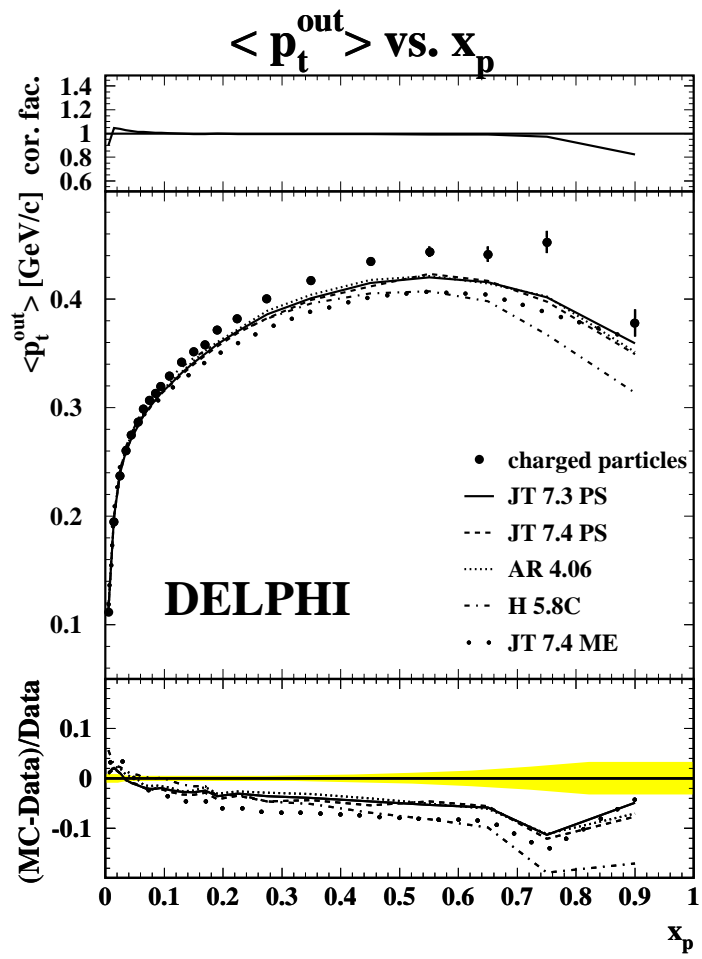

Figure $9:<p_{t}^{\text {out }}>$ vs. $x_{p}$

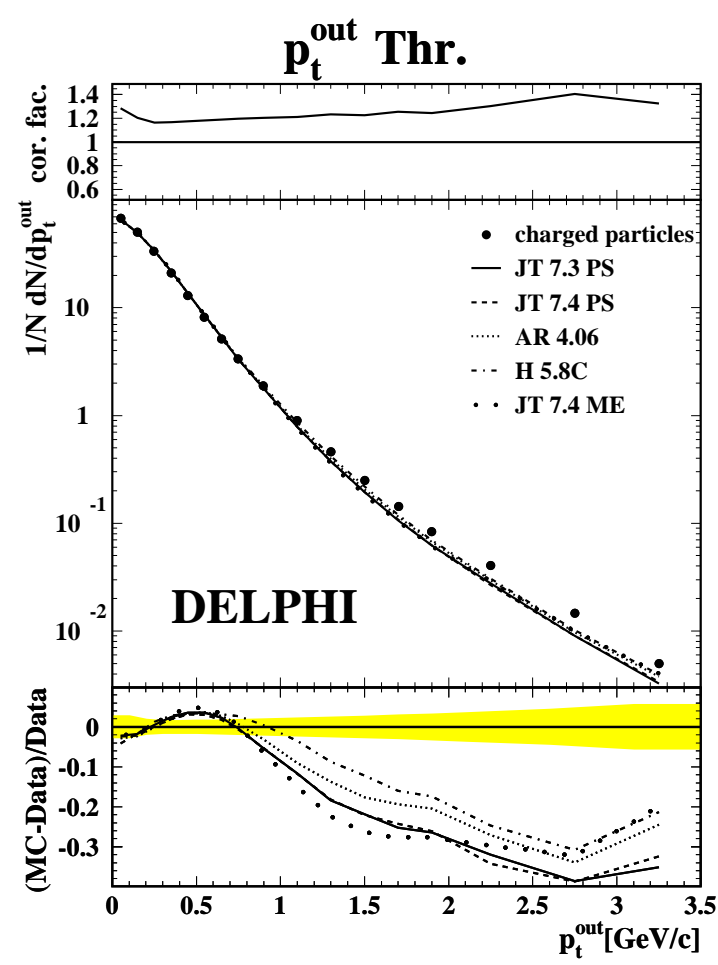

Figure 8: Transverse momentum, $p_{t}^{\text {out }}$, with respect to the Thrust axis

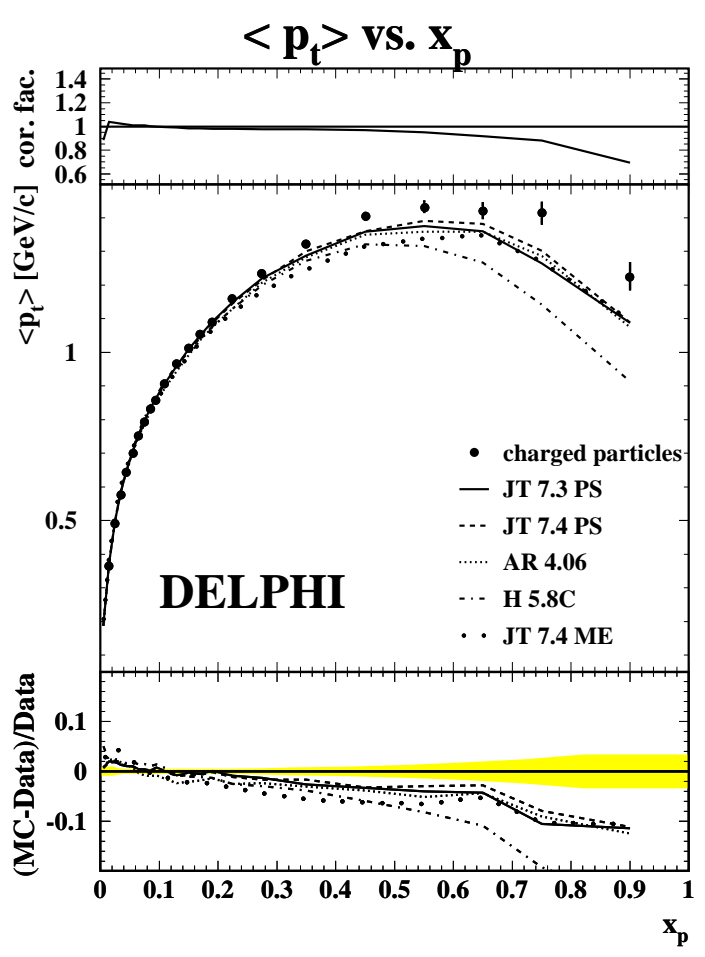

Figure 10: $<p_{t}>$ vs. $x_{p}$ 
F.2 Event Shape Distributions
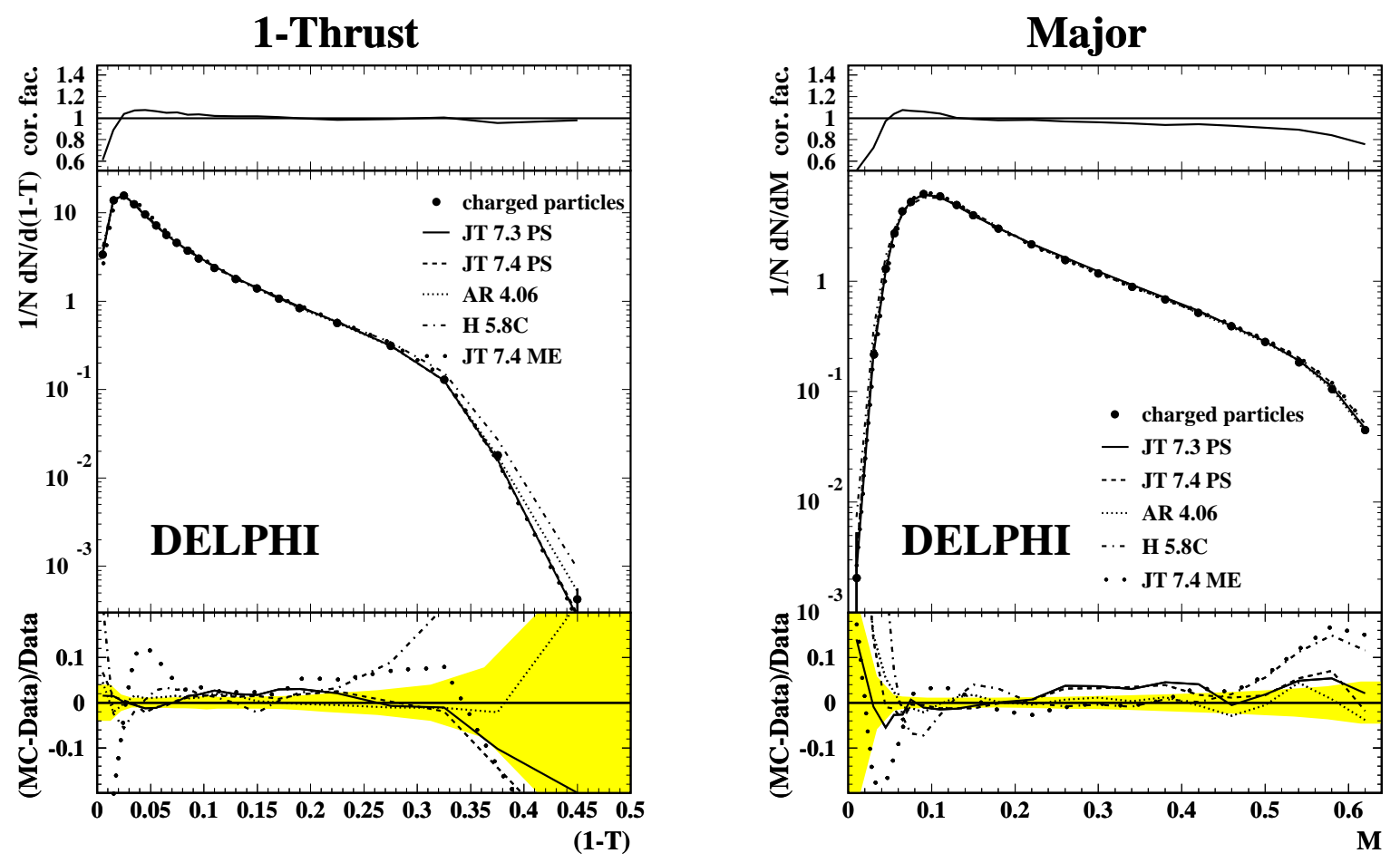

Figure 11: 1-Thrust, $1-T$
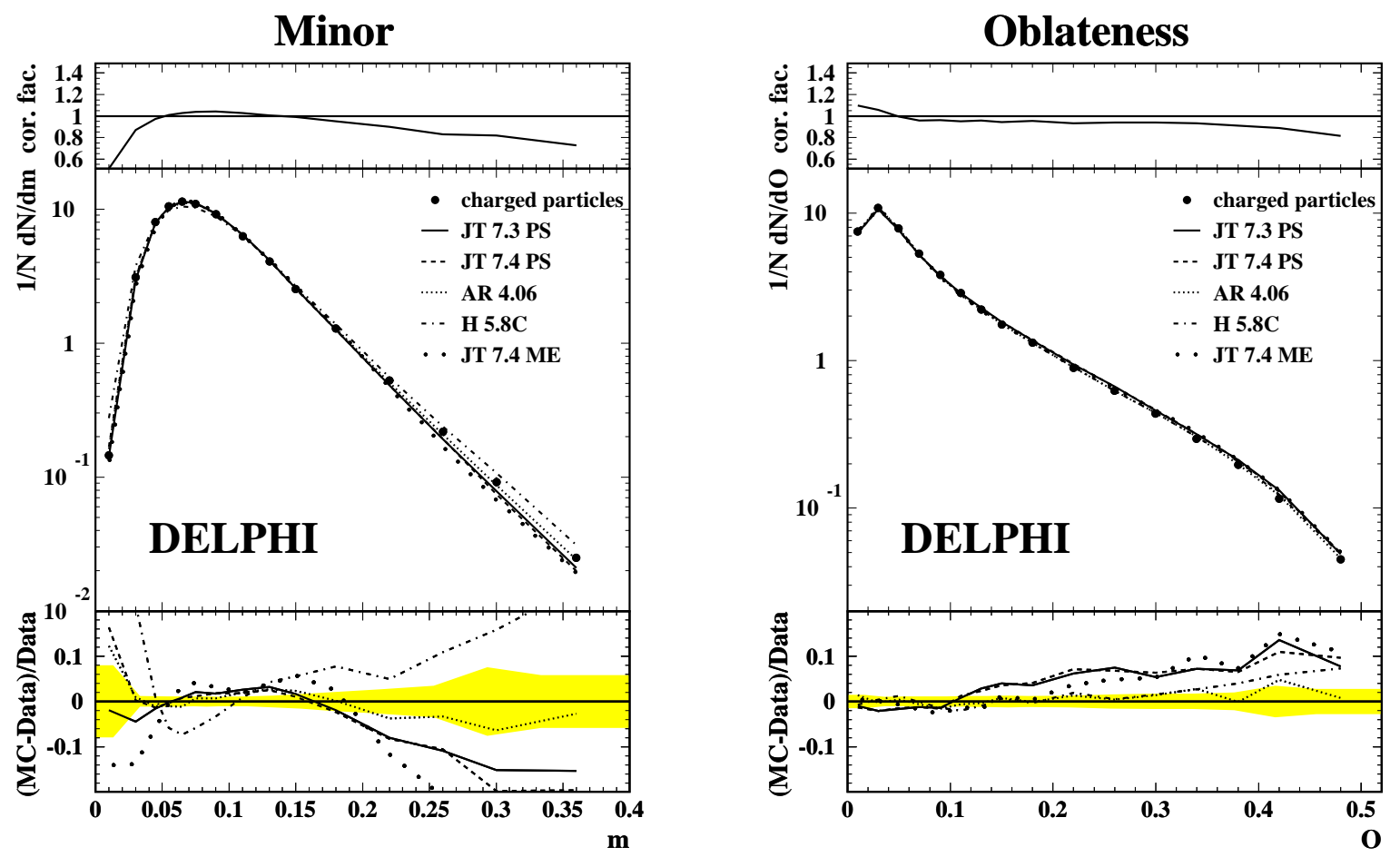

Figure 13: Minor, $m$

Figure 14: Oblateness, $O$ 


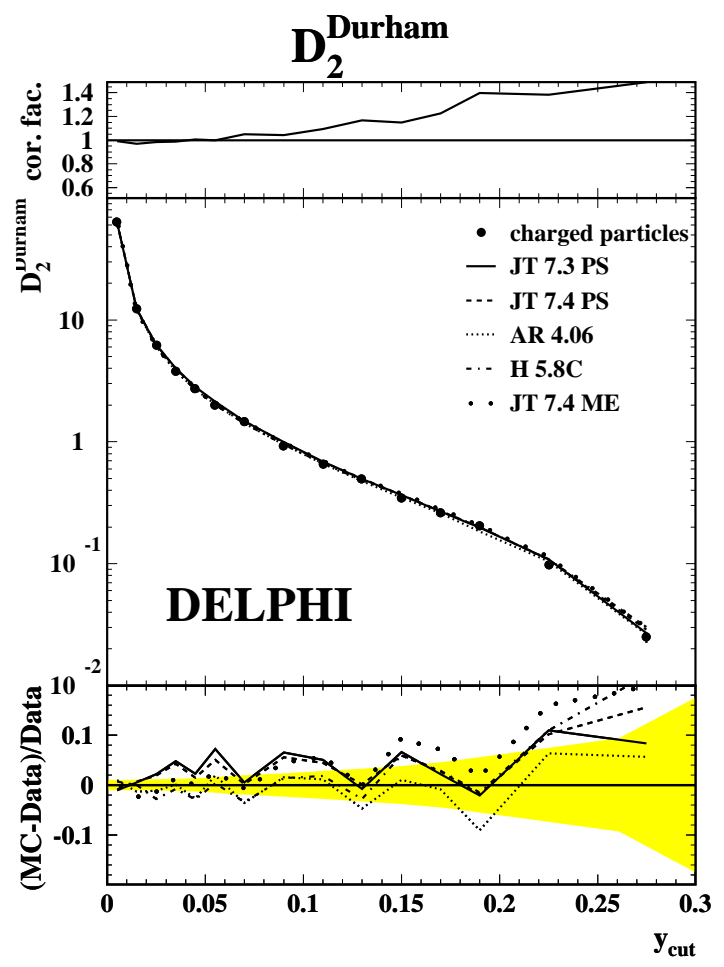

Figure 15: Differential 2-jet rate for the Durham Algorithm, $D_{2}^{\text {Durham }}$

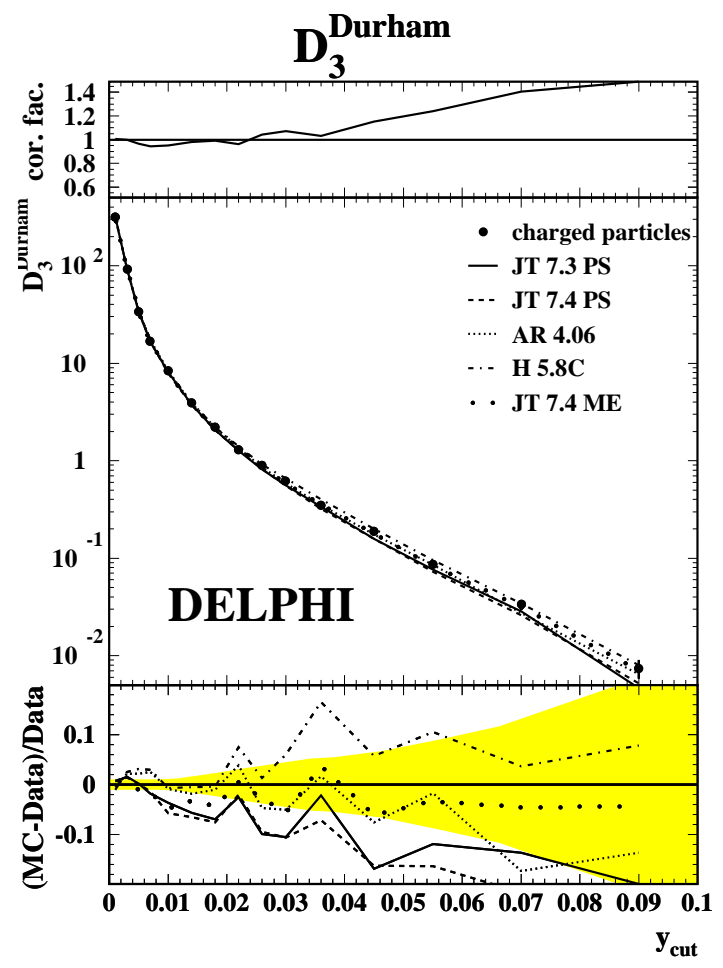

Figure 17: Differential 3-jet rate for the Durham Algorithm, $D_{3}^{\text {Durham }}$

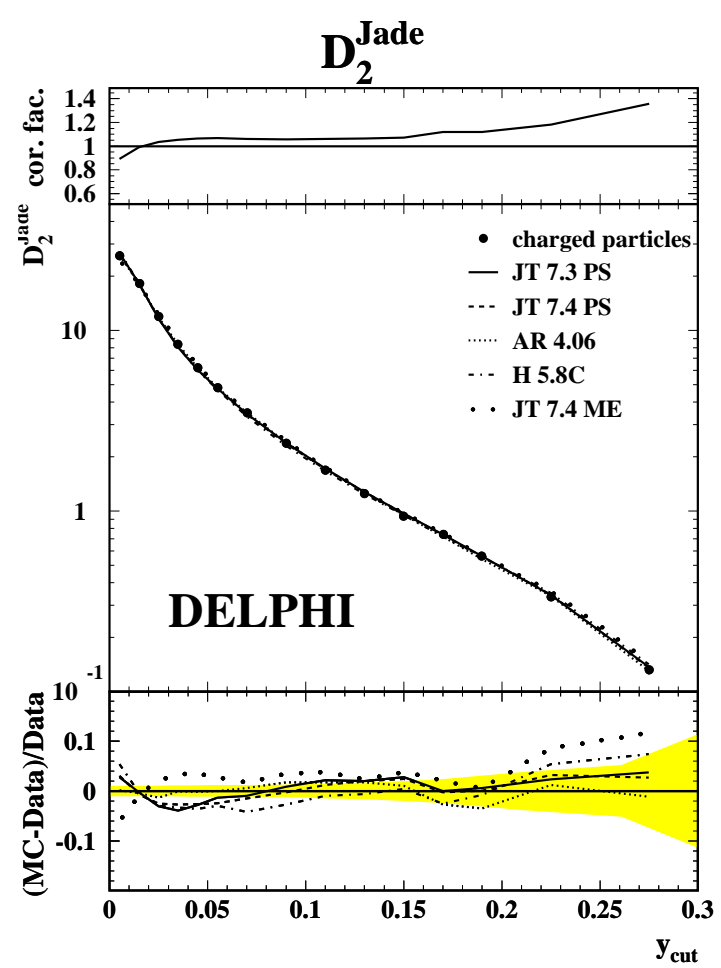

Figure 16: Differential 2-jet rate for the Jade Algorithm, $D_{2}^{\text {Jade }}$

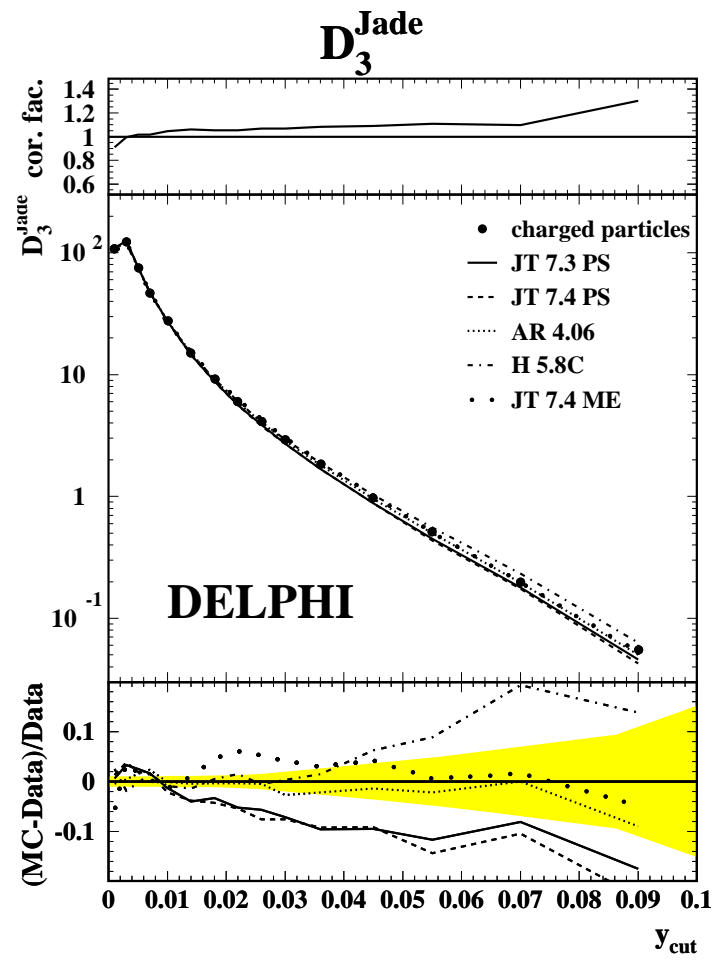

Figure 18: Differential 3-jet rate for the Jade Algorithm, $D_{3}^{J a d e}$ 


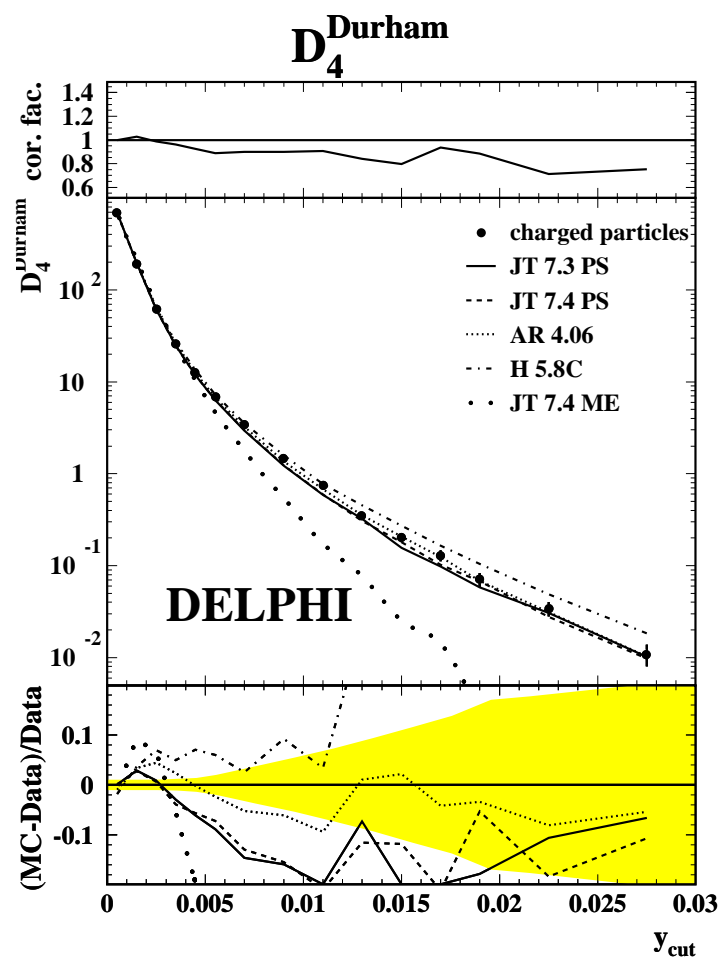

Figure 19: Differential 4-jet rate for the Durham Algorithm, D Durham

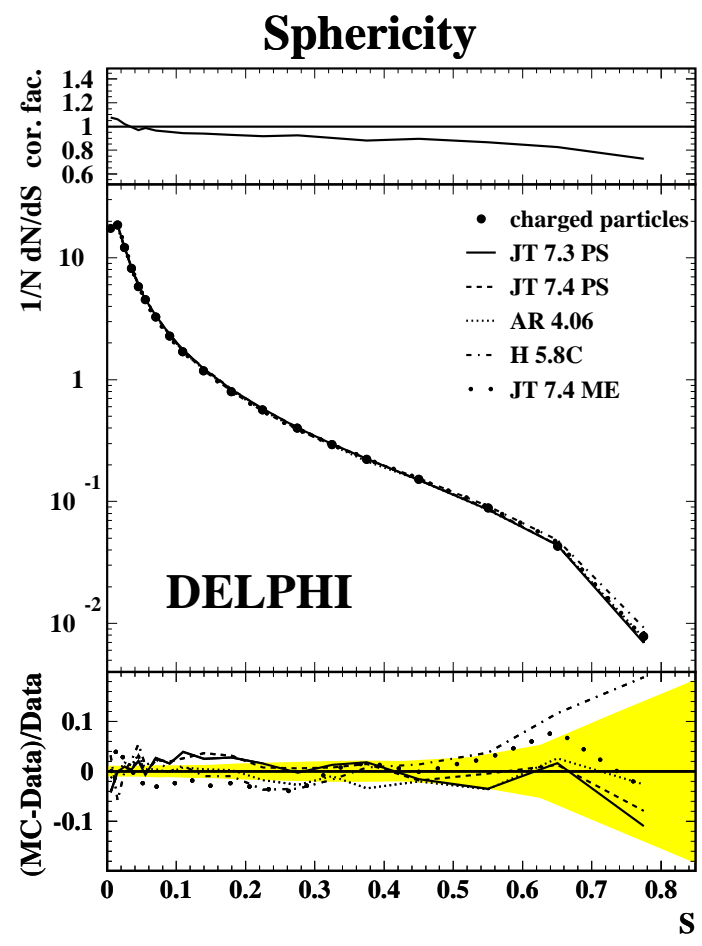

Figure 21: Sphericity, $S$

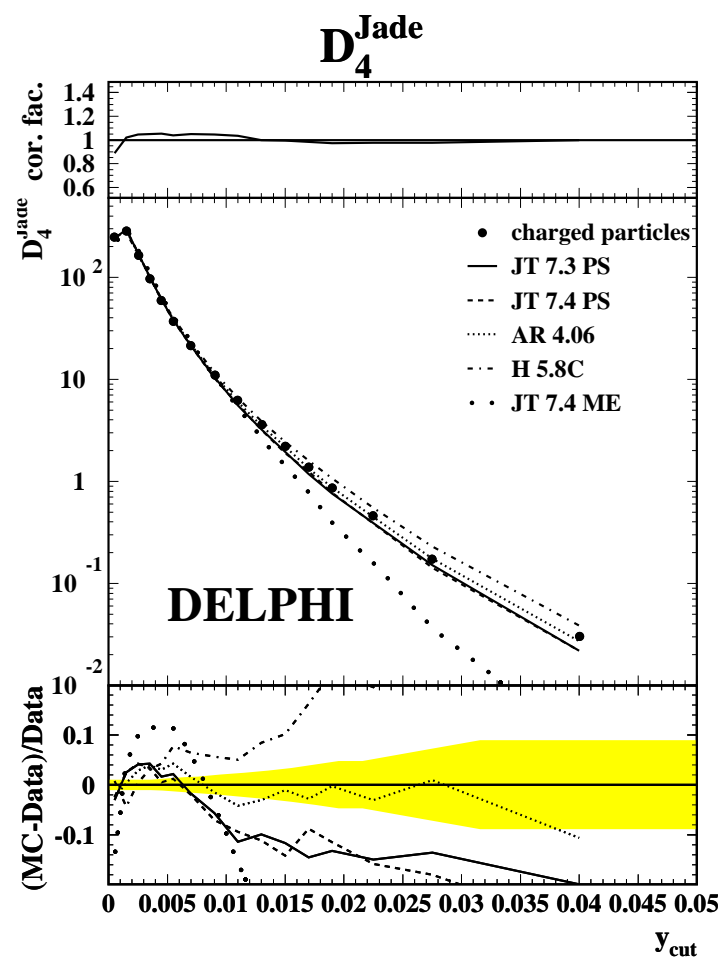

Figure 20: Differential 4-jet rate for the Jade Algorithm, $D_{4}^{\text {Jade }}$

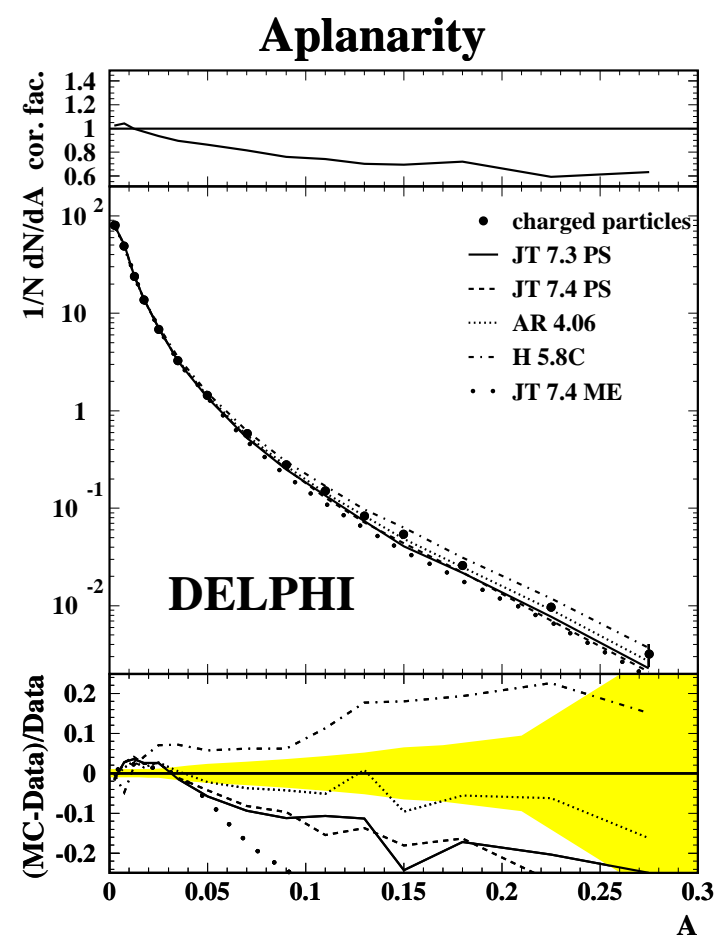

Figure 22: Aplanarity, $A$ 


\section{Planarity}

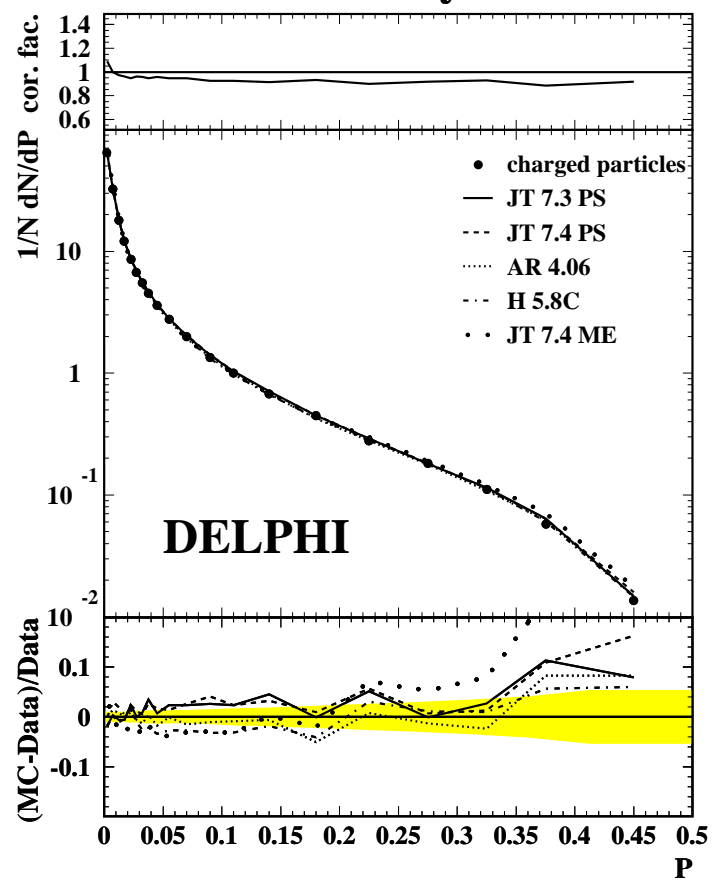

Figure 23: Planarity, $P$

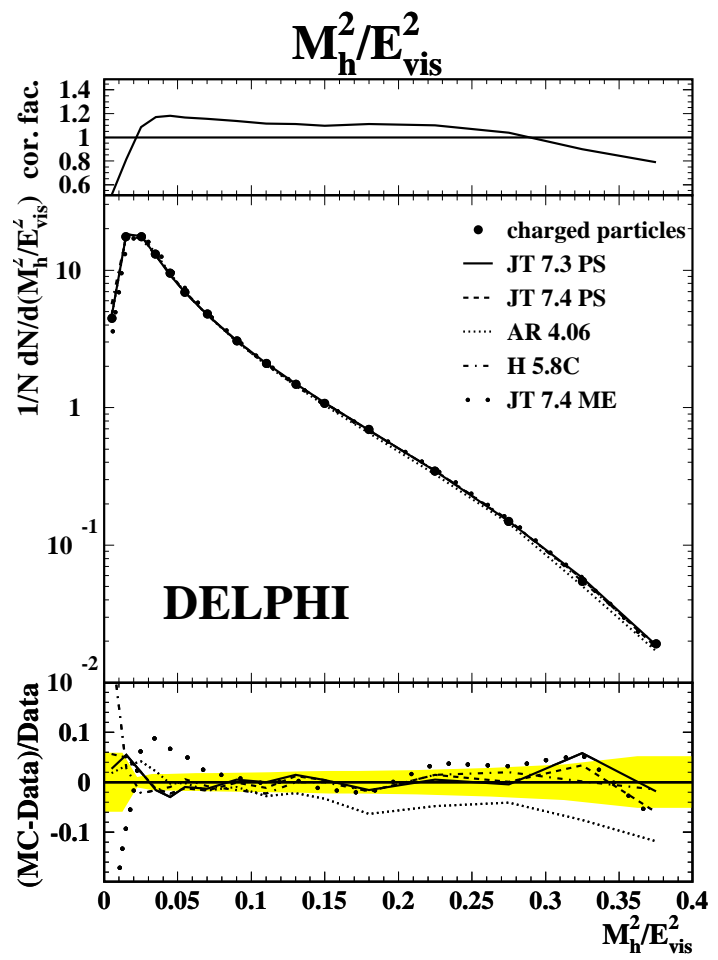

Figure 25: Heavy Hemisphere Mass, $M_{h}^{2} / E_{v i s}^{2}$

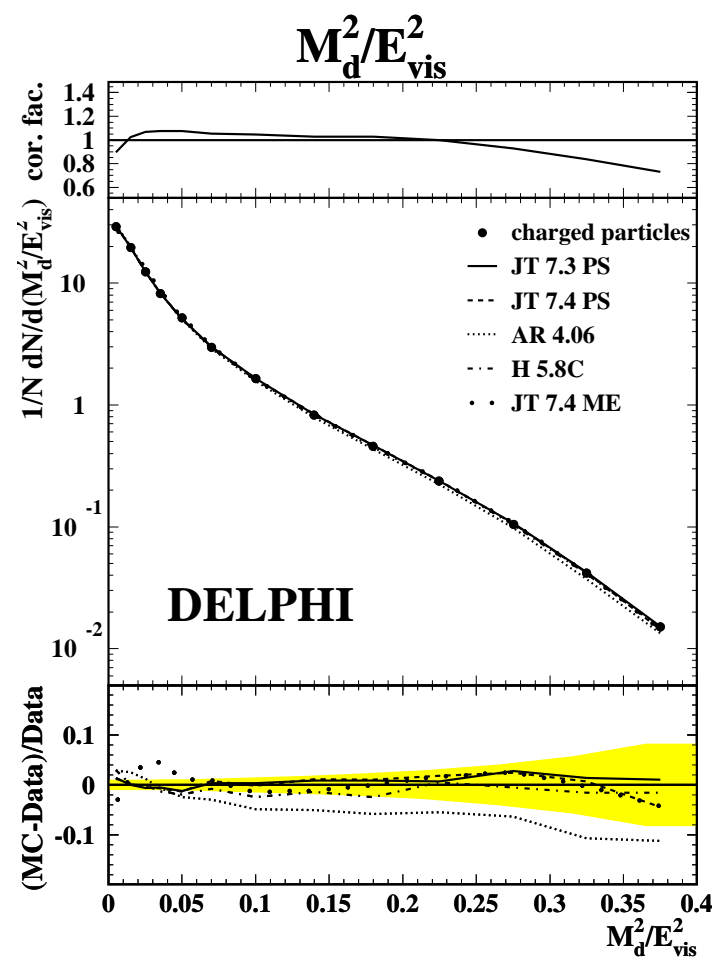

Figure 24: Difference of Hemisphere masses, $M_{d}^{2} / E_{v i s}^{2}$

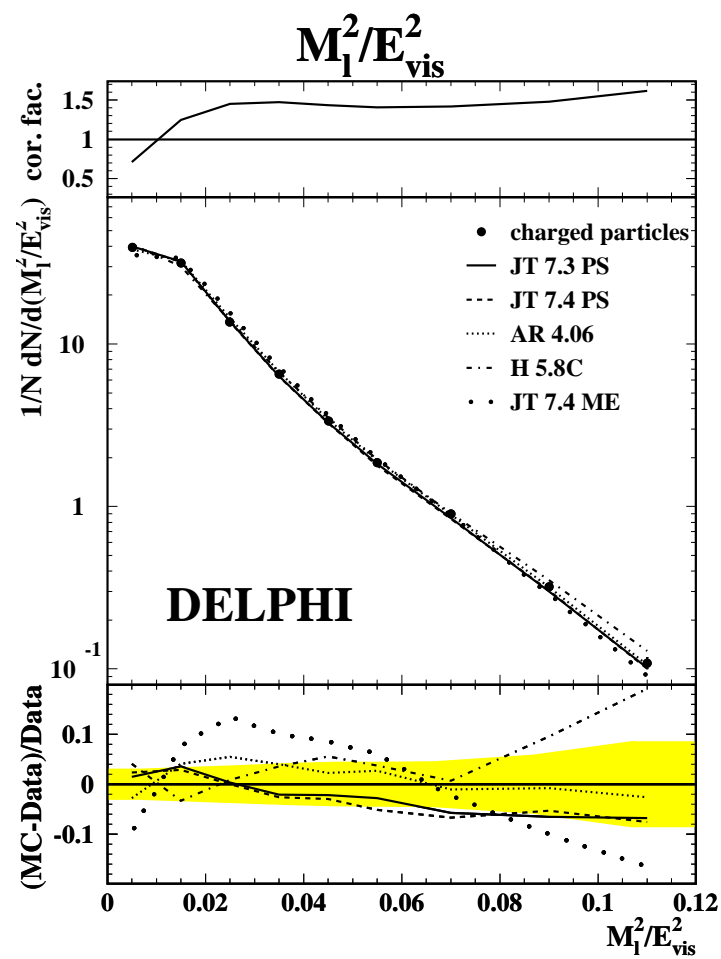

Figure 26: Light Hemisphere Mass, $M_{l}^{2} / E_{v i s}^{2}$ 


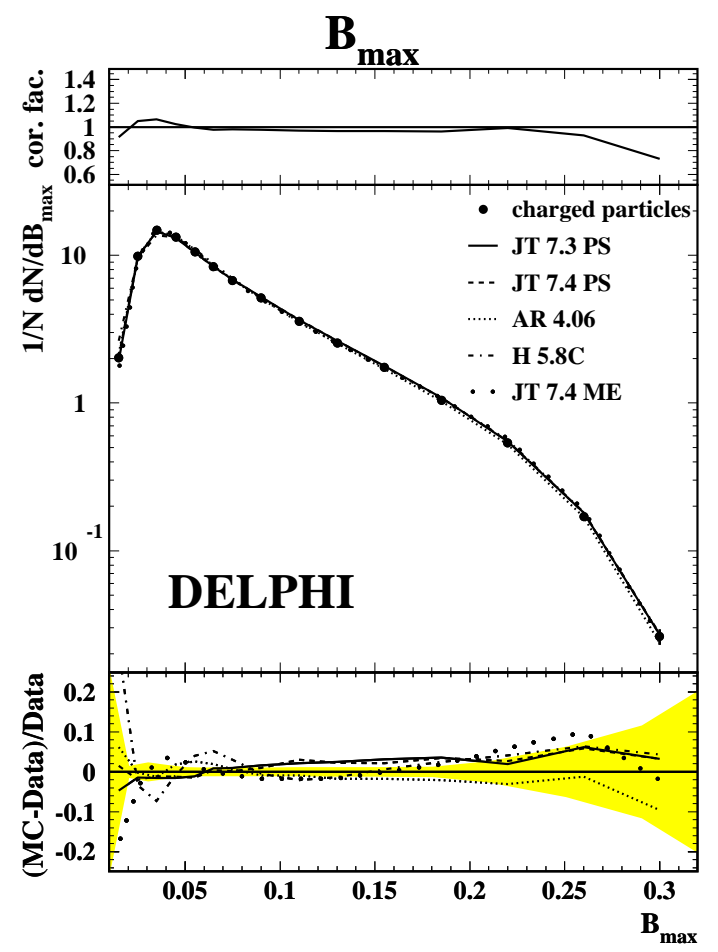

Figure 27: Wide Hemisphere Broadening, $B_{\max }$

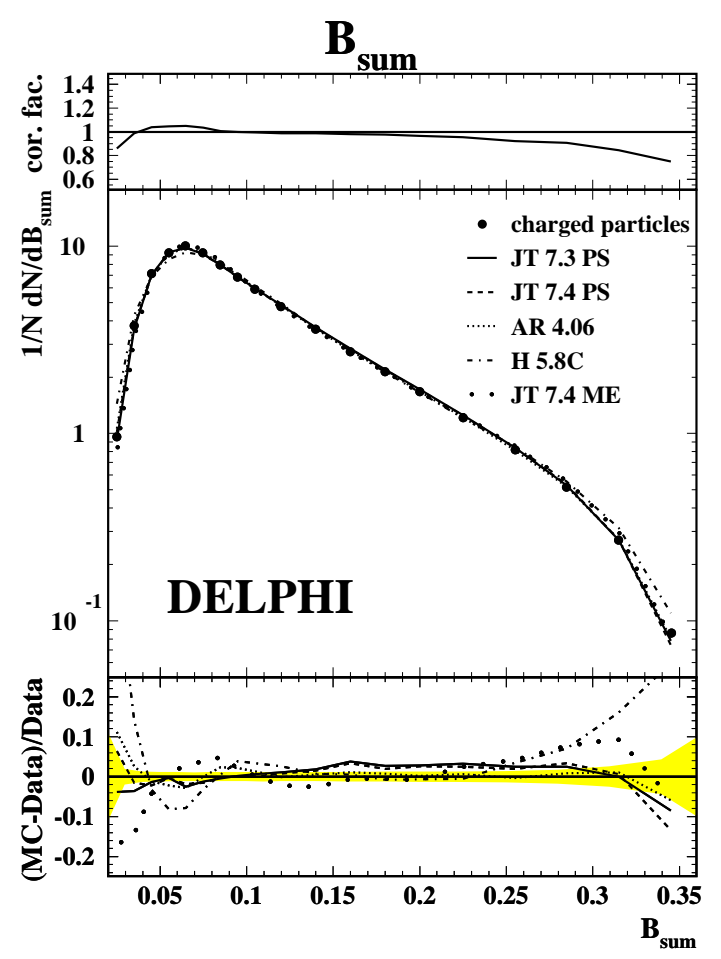

Figure 29: Total Hemisphere Broadening, $B_{\text {sum }}$

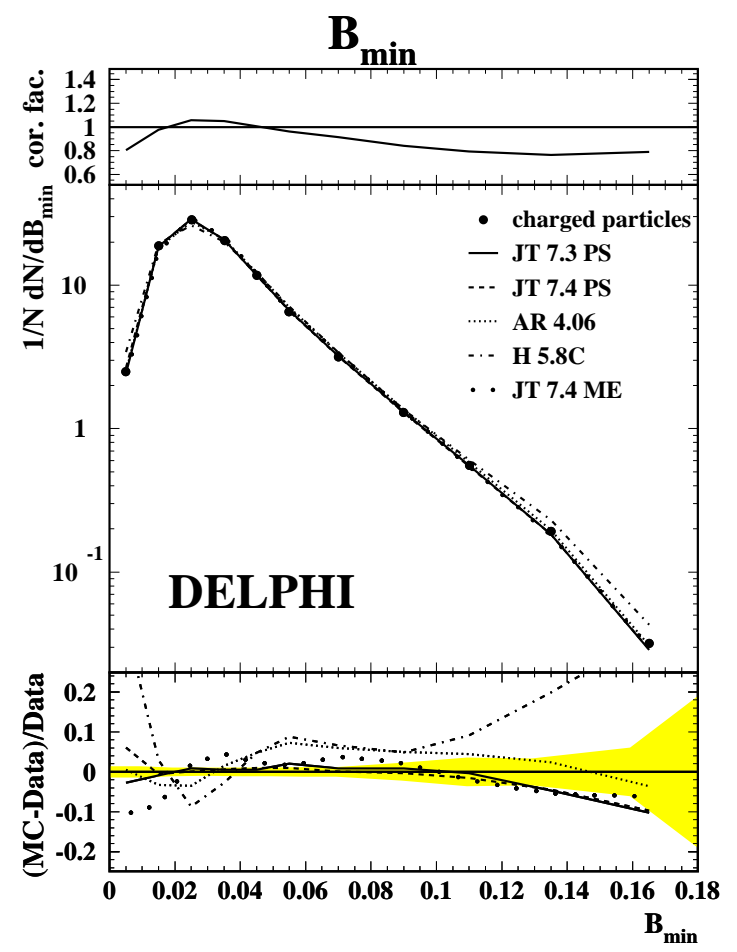

Figure 28: Narrow Hemisphere Broadening, $B_{\min }$

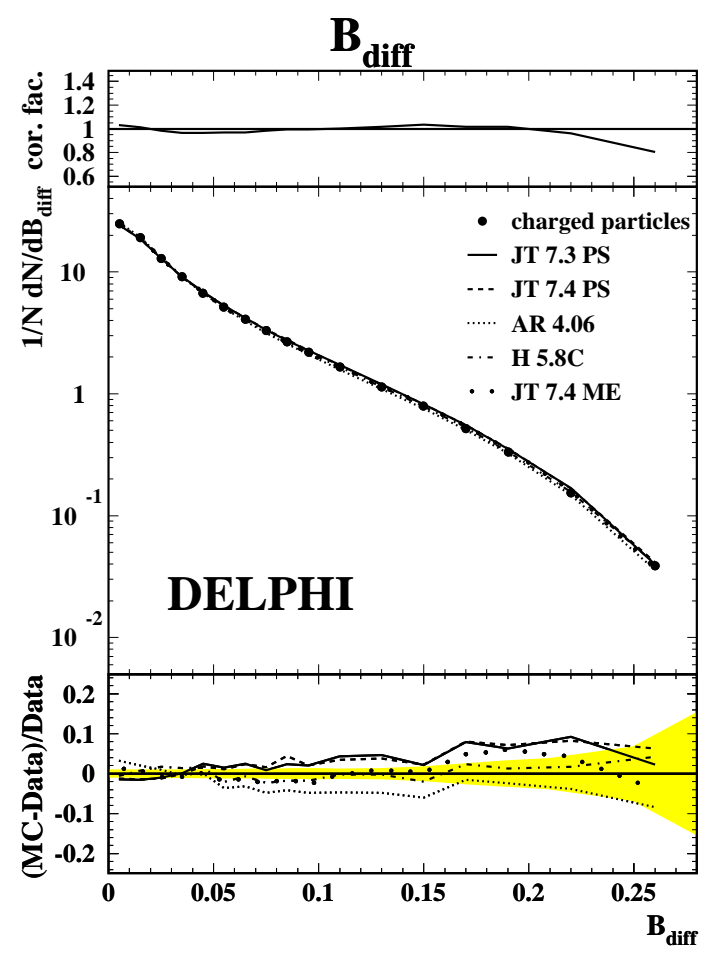

Figure 30: Difference of the Hemisphere Broadenings, $B_{\text {diff }}$ 


\section{C-Parameter}

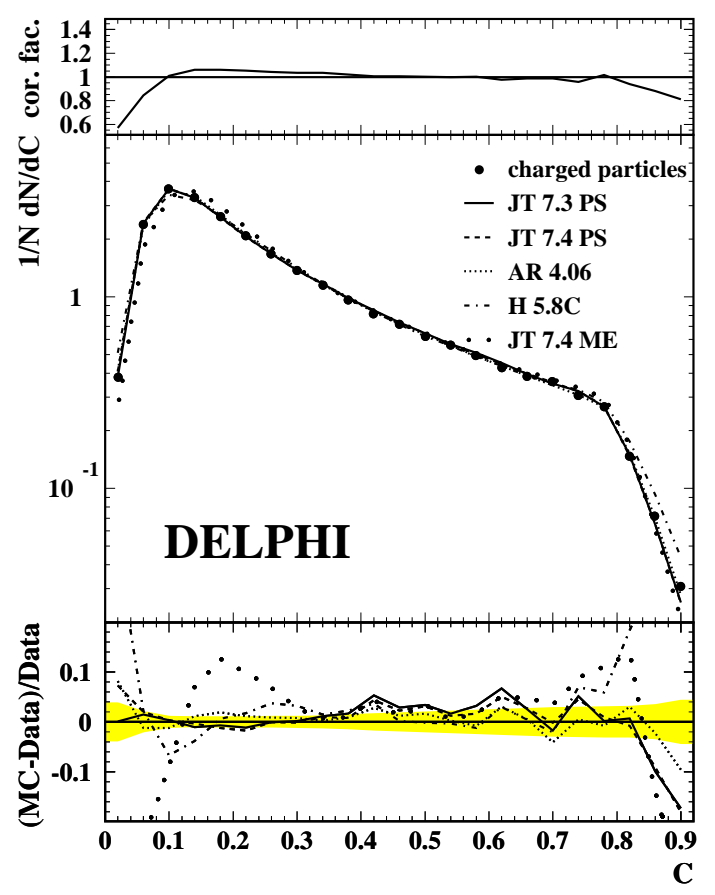

Figure 31: C-Parameter, $C$

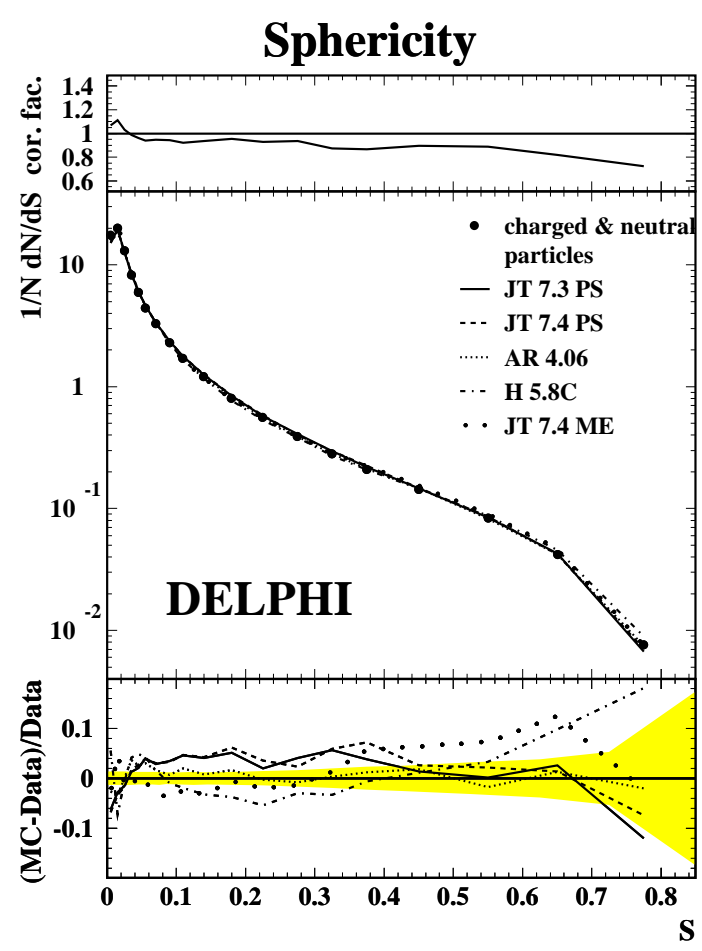

Figure 33: Sphericity, $S$, measured from charged and neutral particles

\section{D-Parameter}

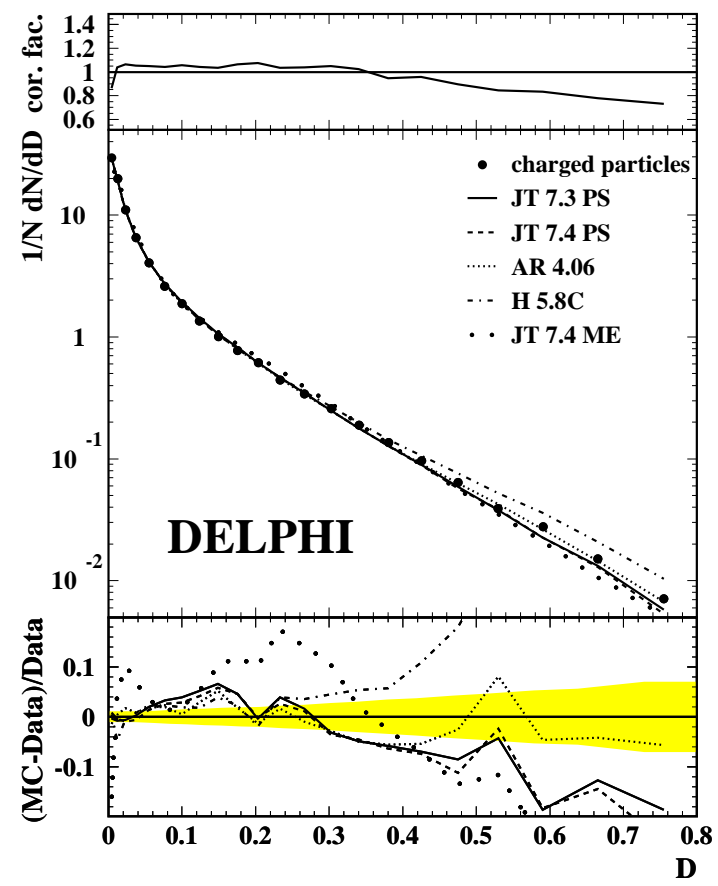

Figure 32: D-Parameter, $D$

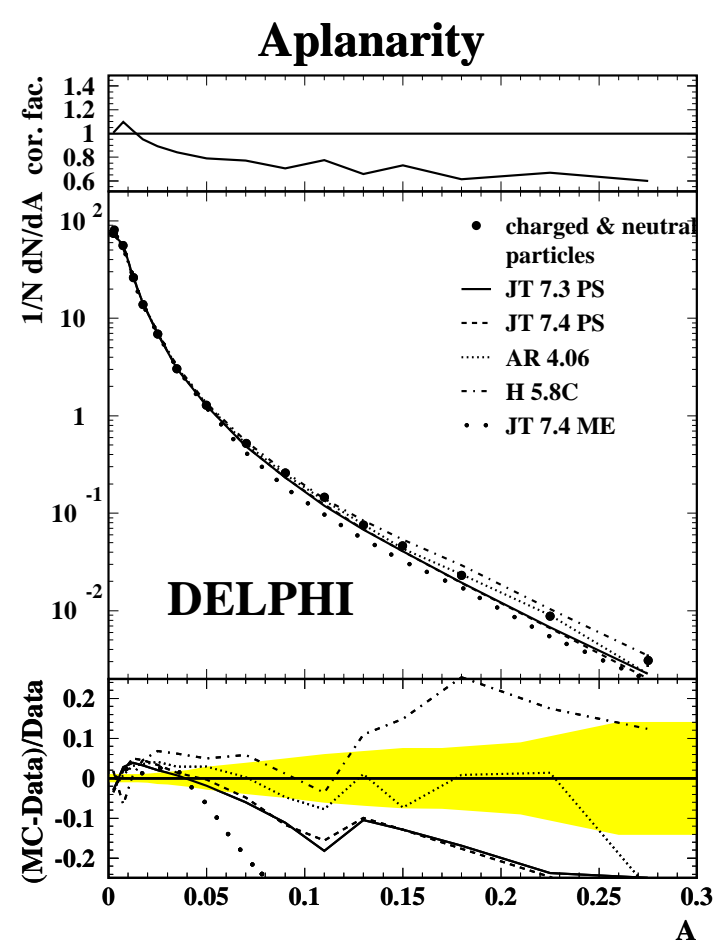

Figure 34: Aplanarity, A, as measured from charged and neutral particles 


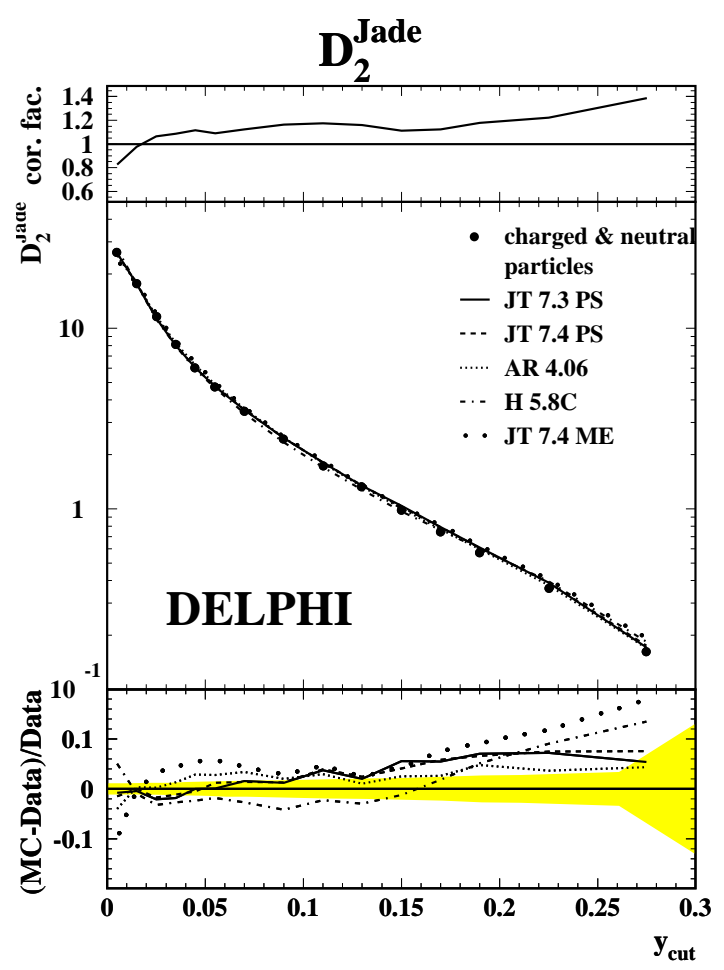

Figure 35: Differential 2-jet rate for the Jade Algorithm, $D_{2}^{J}$, measured from charged and neutral particles

\section{EEC}

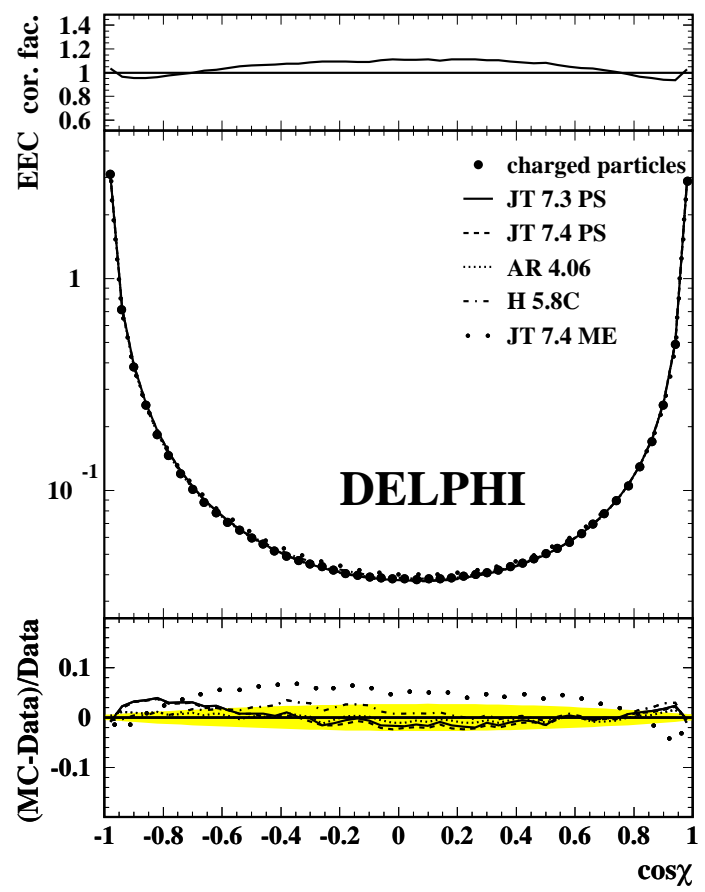

Figure 37: Energy Energy

Correlation, EEC

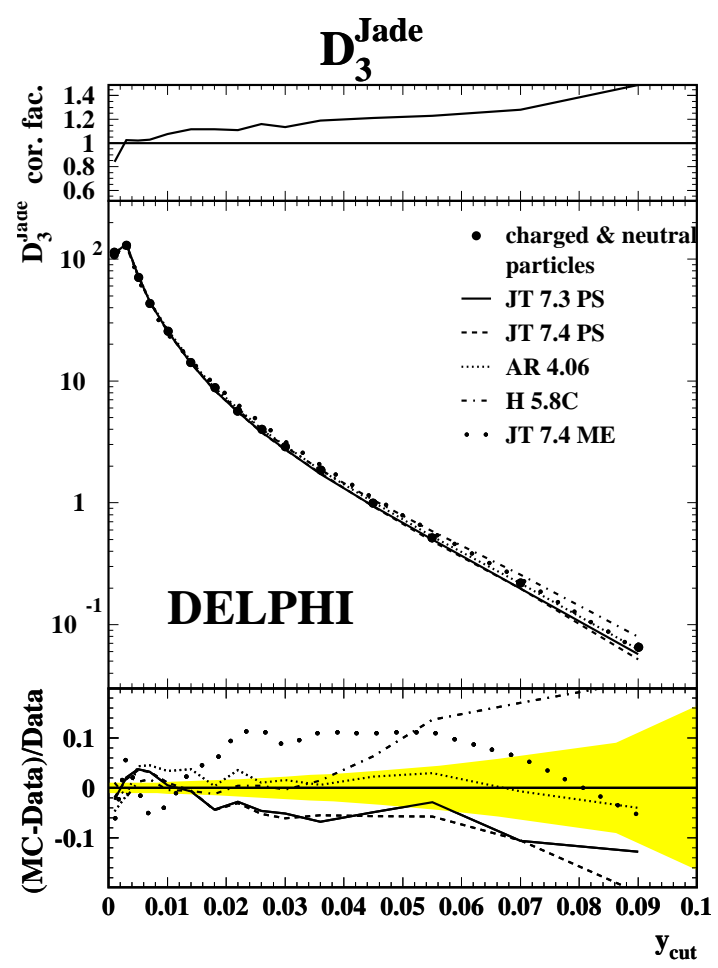

Figure 36: Differential 3-jet rate for the Jade Algorithm, $D_{3}^{J}$, measured from charged and neutral particles

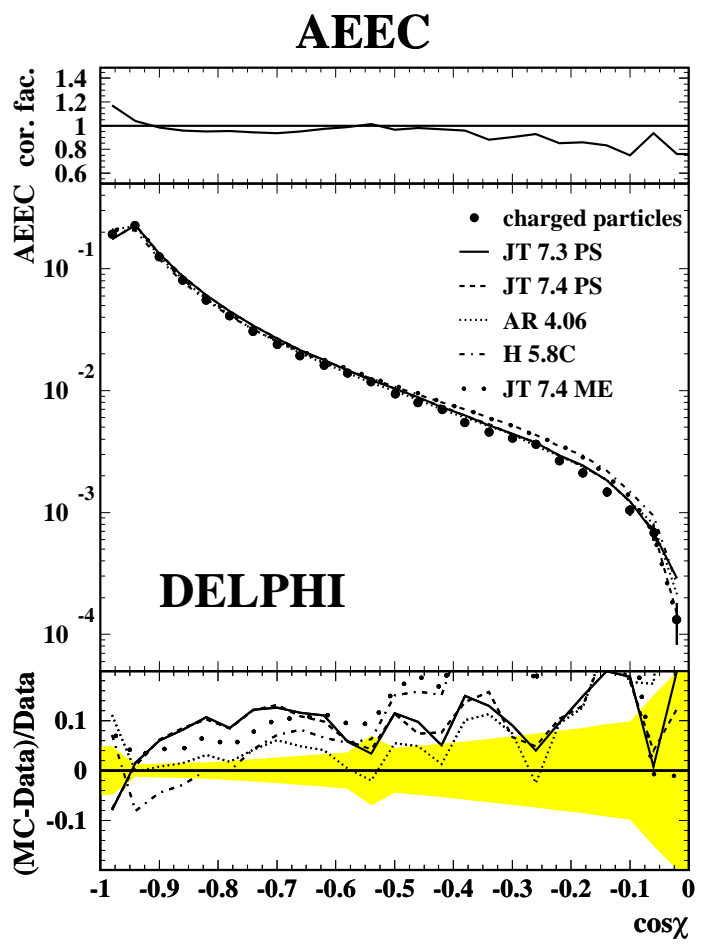

Figure 38: Asymmetry of the Energy Energy Correlation, AEEC 


\section{F.3 Identified Particle Distributions}
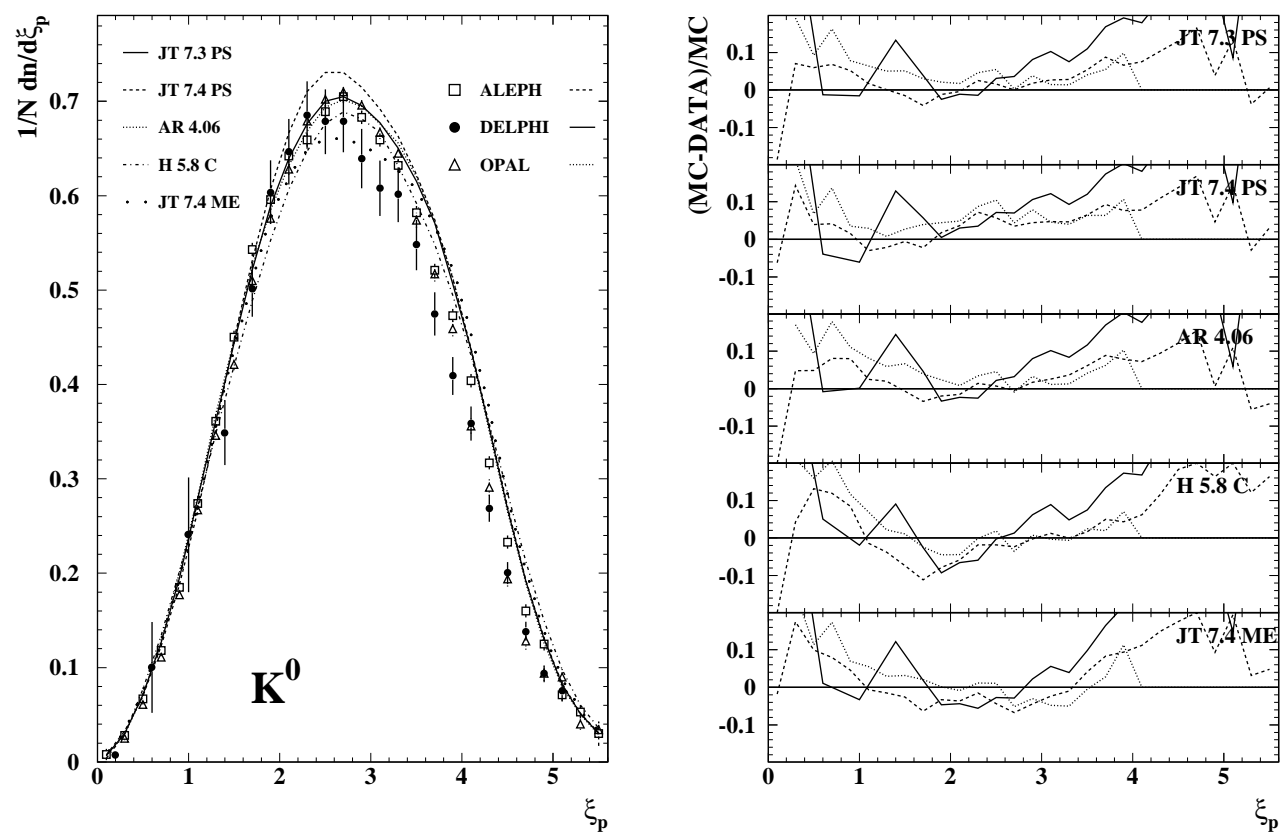

Figure 39: $\xi_{p}$ distribution of $K^{0}$. The curves on the right refer to the different experiments given on the left plot.
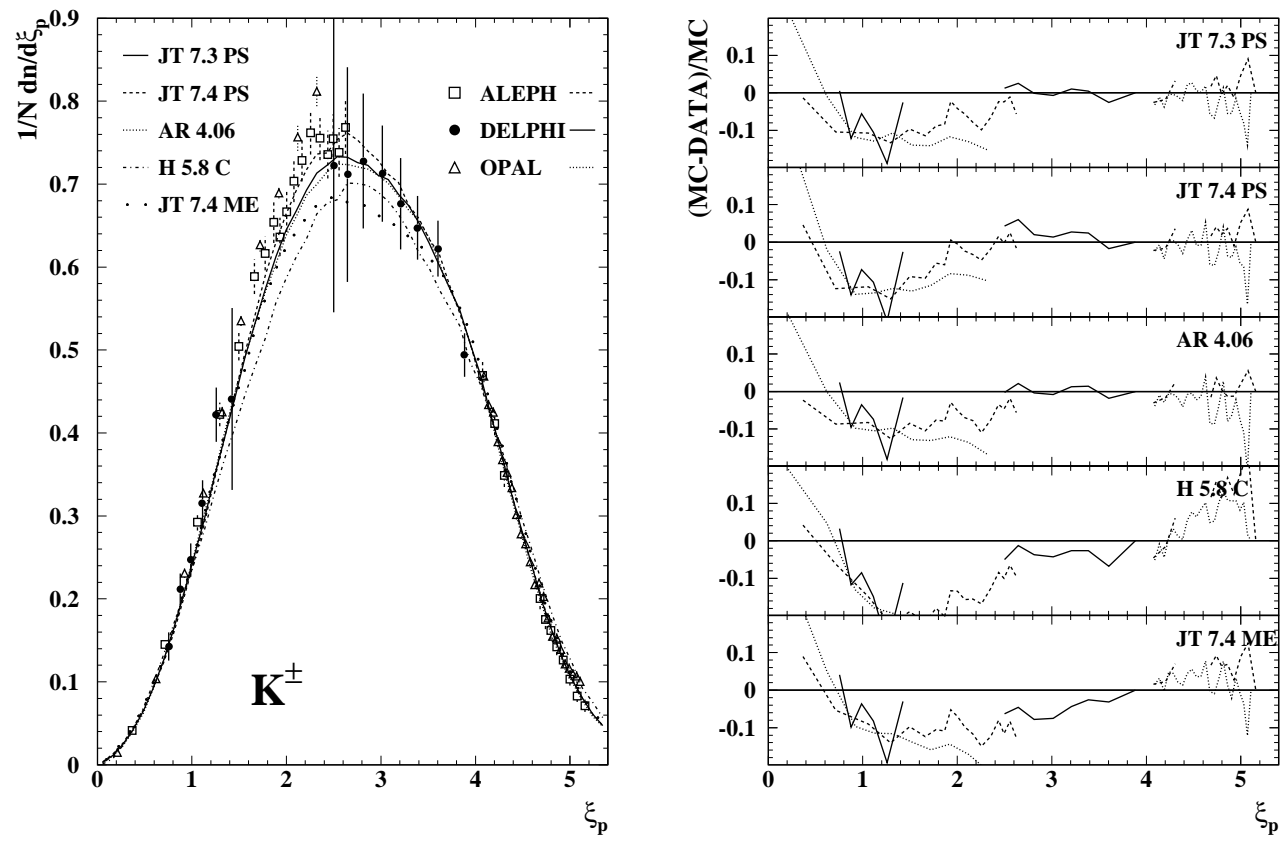

Figure 40: $\xi_{p}$ distribution of $K^{ \pm}$. The curves on the right refer to the different experiments given on the left plot. 


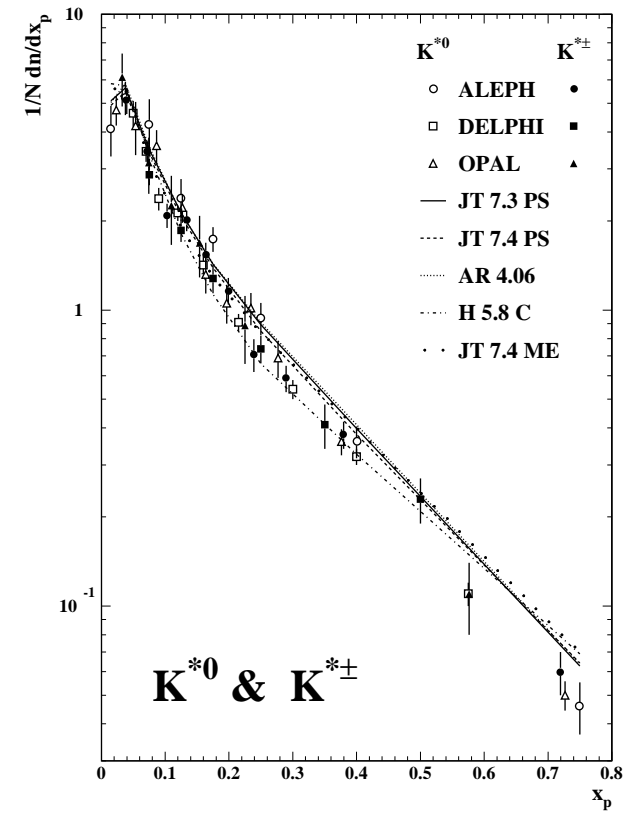

Figure 41: $x_{E}$ distribution of $K^{* 0}$ and $K^{* \pm}$

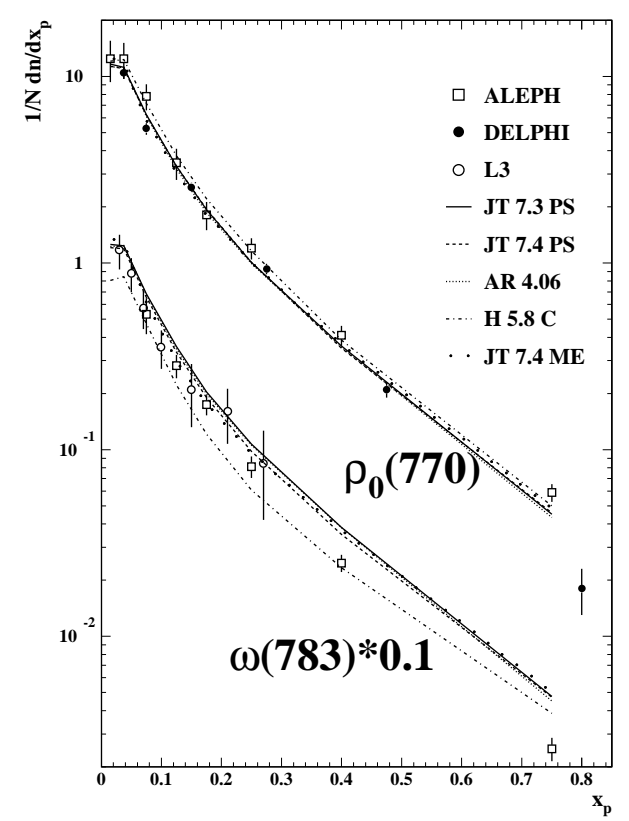

Figure 43: $x_{p}$ distribution of $\rho$ and $\omega$

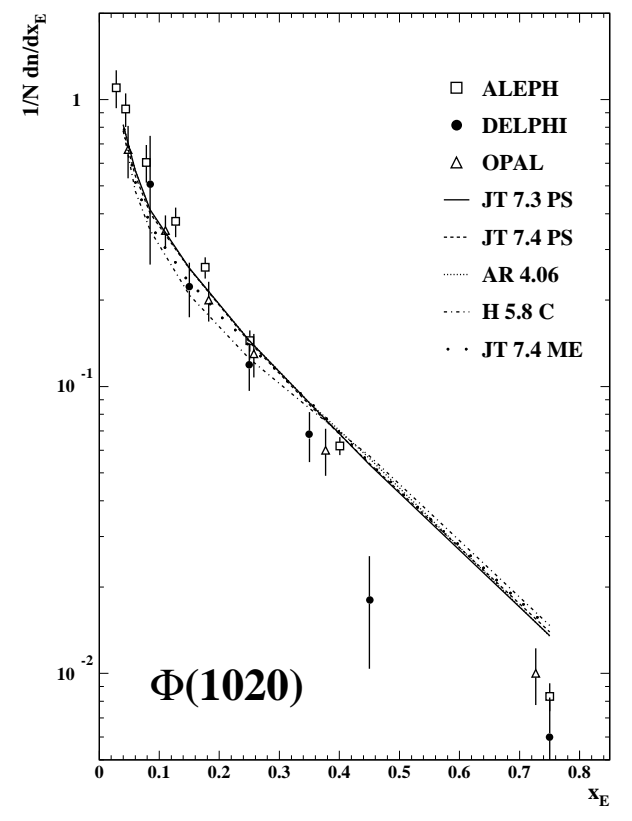

Figure 42: $x_{E}$ distribution of $\phi$

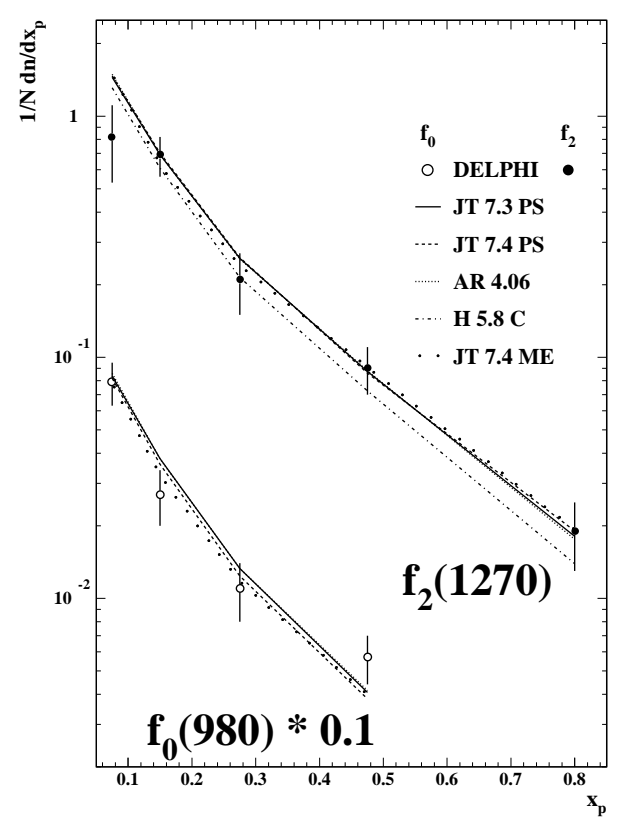

Figure 44: $x_{p}$ distribution of $f_{0}(980)$ and $f_{2}(1270)$ 

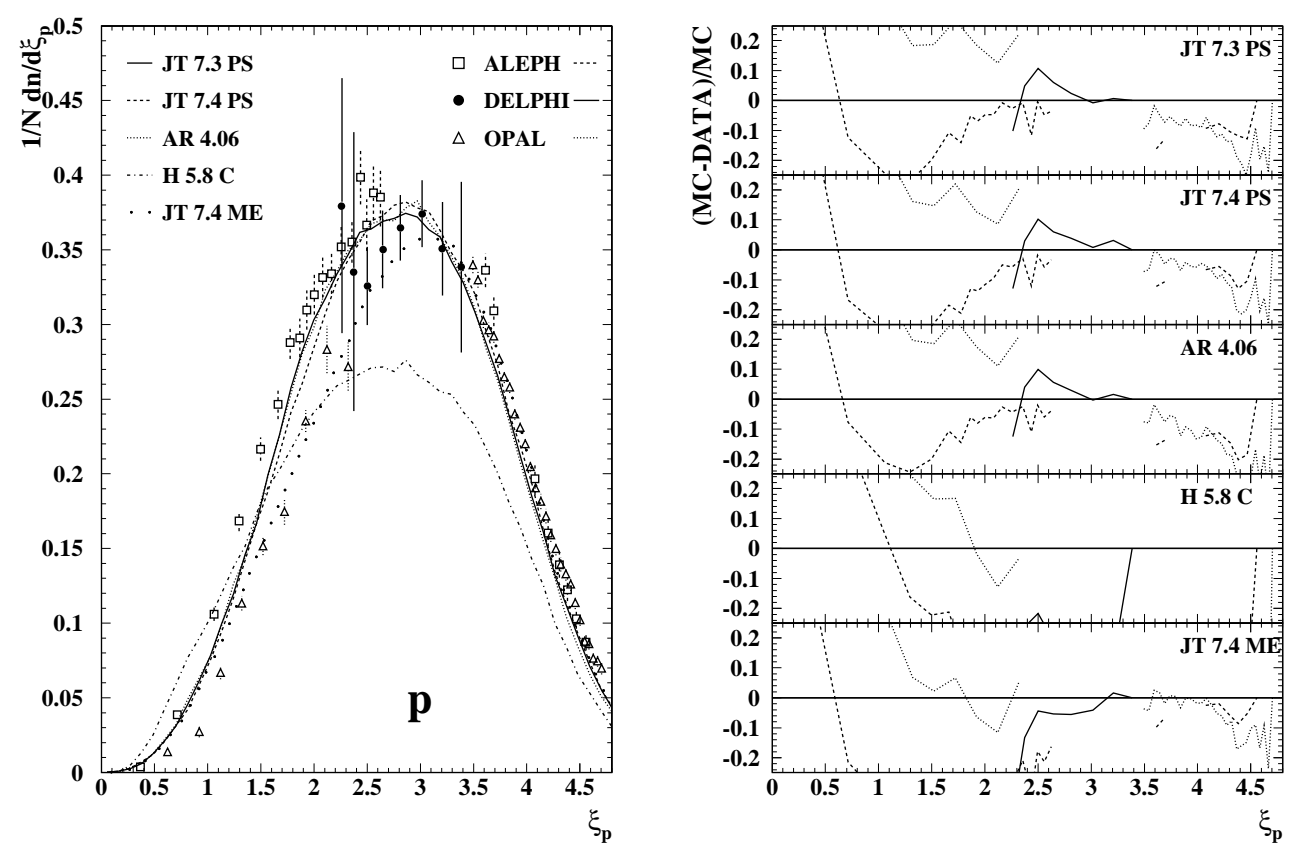

Figure 45: $\xi_{p}$ distribution of protons. The curves on the right refer to the different experiments given on the left plot.
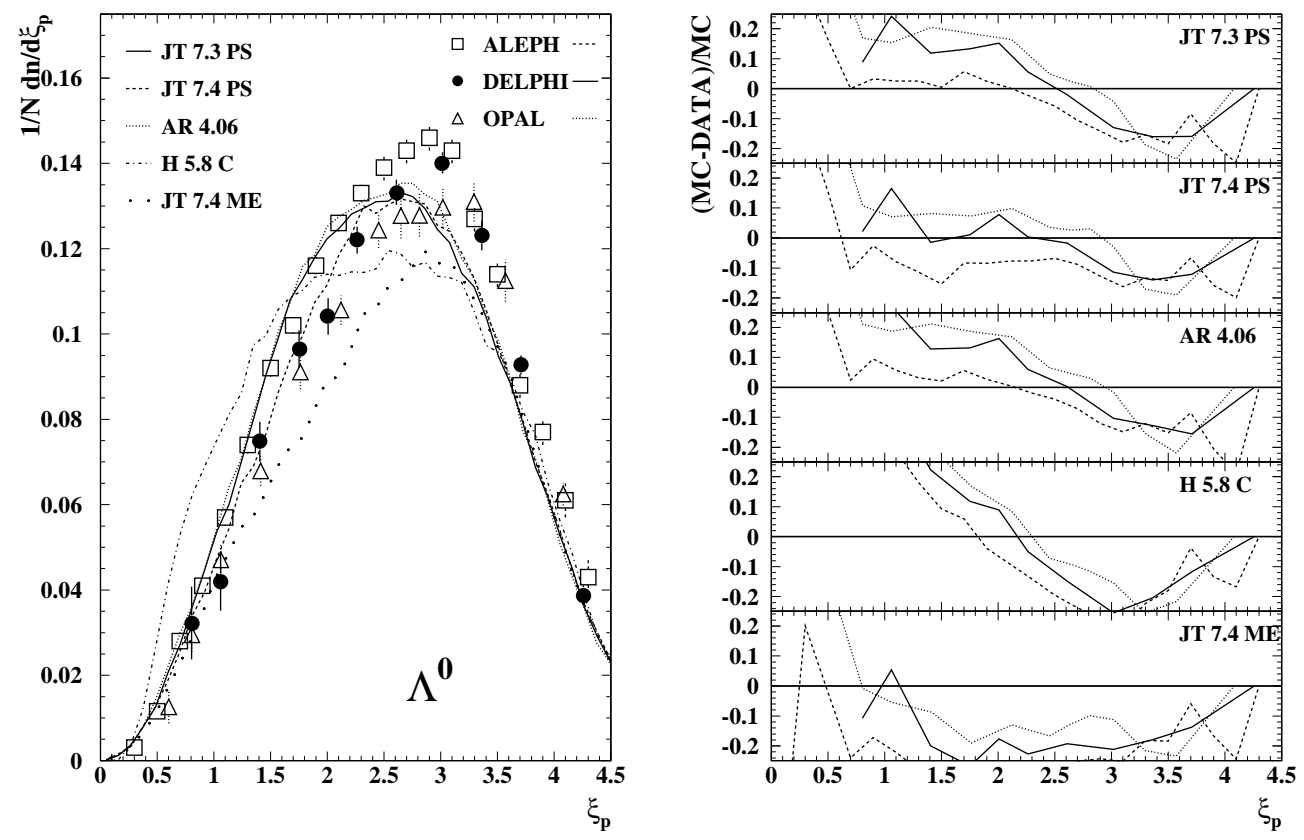

Figure 46: $\xi_{p}$ distribution $\Lambda^{0}$. The curves on the right refer to the different experiments given on the left plot. 

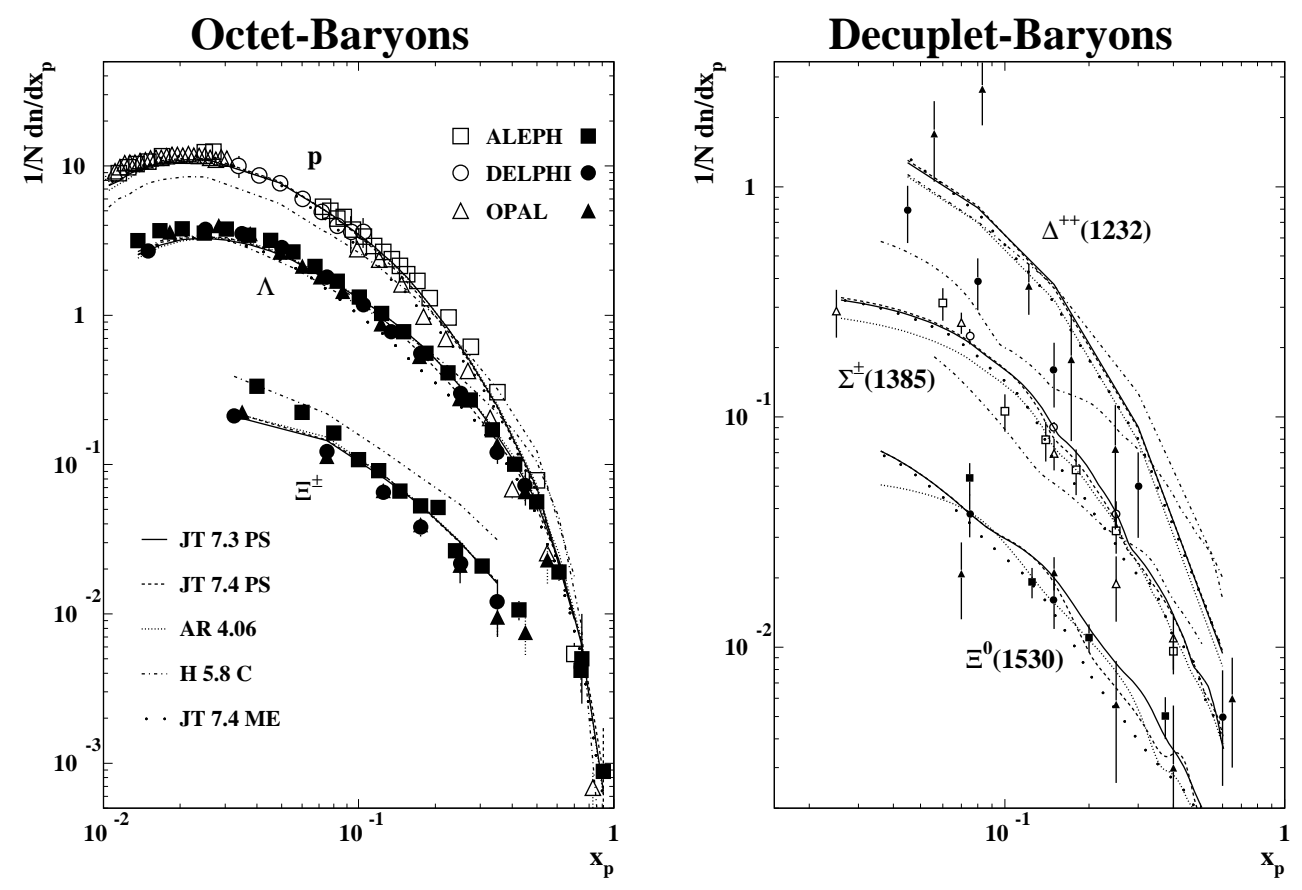

Figure 47: $x_{p}$ distributions of octet and decuplet baryons 


\section{References}

[1] DELPHI Coll., P. Aarnio et al., Nucl. Inst. Methods A303 (1991) 187.

[2] DELPHI Coll., P. Abreu et al., Nucl. Inst. Methods A378 (1996) 57.

[3] F. Jadach, B.F.L. Ward, Z. Was, Comp. Phys. Comm. 40 (1986) 285; Nucl. Phys. B253 (1985) 441.

[4] T. Sjöstrand, Comp. Phys. Comm. 39 (1986) 347;

T. Sjöstrand and M. Bengtsson, Comp. Phys. Comm. 46 (1987) 367.

[5] J.E. Campagne and R. Zitoun, Z. Phys. C43 (1989) 469.

[6] V. Blobel, 'Unfolding methods in high energy physics experiments',

Lecture given at the 1984 School of Computing, DESY 84-118;

V.B. Anikeev, V.P. Zhigunov, Phys. of Part. and Nucl. 24 (1993) 424.

[7] H. Fürstenau, Ph. D. Thesis, Univ. Karlsruhe, IEKP-KA 92-16, Karlsruhe (1992).

[8] I. G. Knowles et al., QCD Event Generators, CERN 96-01 Vol. 2.

[9] L. Lönnblad, Comp. Phys. Comm. 71 (1992) 15.

[10] G. Marchesini et al., Comp. Phys. Comm. 67 (1992) 465.

[11] C. Peterson, D. Schlatter, I. Schmitt and P. Zerwas, Phys. Rev. D27 (1983) 105.

[12] B. Andersson, G. Gustafson, G. Ingelman, T. Sjöstrand, Phys. Rep. 97 (1983) 31.

[13] ALEPH Coll., D. Busculic et al., Z. Phys. C64 (1994) 361.

[14] M. Weierstall, Dissertation, Bergische Univ. - GH Wuppertal, WUB-DIS 95-11, Wuppertal (1995).

[15] K. Münich, Diplomarbeit, Bergische Univ. - GH Wuppertal, WUD 95-5, Wuppertal (1995).

[16] F. Gutbrod, G. Kramer, G. Schierholz, Z. Phys. C21 (1984) 235;

K. Fabricius et al., Z. Phys. C11 (1981) 315.

[17] R.K. Ellis, D.A. Ross, E.A. Terrano, Phys. Rev. Lett. 45 (1980) 1225,

Nucl. Phys. B178 (1981) 421.

[18] compare discussion and references in: QCD Generators, B. Bambah et al. CERN 89-08 Vol. 3.

[19] P.M. Stevenson, Phys. Rev. D23 (1981) 2916.

[20] G. Gustavson, U. Petterson, Nucl. Phys. B308 (1988) 746.

[21] TASSO Coll., M. Althoff et al., Z. Phys. C26 (1984) 157;

TASSO Coll., W. Braunschweig et al. Z. Phys. C41 (1988) 359.

[22] ALEPH Coll., D. Decamp et al., Z. Phys. C55 (1992) 209.

[23] L3 Coll., B. Adeva et al., Z. Phys. C55 (1992) 39.

[24] W.H. Press, B.P. Flannery, S.A. Teukolsky, W.T. Vetterling, Numerical Recipes, The Art of Scientific Computing, Cambridge University Press.

[25] NAG library MK16, NAG Ltd., Oxford, GB.

[26] F. James, M. Goossens, MINUIT, Function Minimization and Error Analysis, Reference Manual, CERN Program Library Long Writeup D506 (1992).

[27] OPAL Coll., M.Z. Akrawy et al., Z. Phys. C47 (1990) 505.

[28] V. Gibson, 'Charm and Beauty Hadron Production at $\sqrt{s} \approx M_{Z}$ ', Proc. XXVII ICHEP Conference, Glasgow 1994, P.J. Bussey and I. G. Knowles (ed.)

[29] L3 Coll., O. Adriani et al., Phys. Lett. B286 (1992) 403.

[30] ALEPH Coll., D. Buskulic et al., Phys. Lett. B292 (1992) 210; ALEPH Coll., contrib. paper gls0535 to ICHEP Conference, Glasgow (1994).

[31] OPAL Coll., R. Akers et al., Z. Phys. C67 (1995) 389.

[32] DELPHI Coll., P. Abreu et al., Phys. Lett. B345 (1995) 598.

[33] ALEPH Coll., D. Buskulic et al., Z. Phys. C66 (1995) 355.

[34] DELPHI Coll., P. Abreu et al., Nucl. Phys. B444 (1995) 3.

[35] DELPHI Coll., P. Abreu et al., Z. Phys. C67 (1995) 543.

[36] OPAL Coll., R. Akers et al., Z. Phys. C63 (1994) 181.

[37] OPAL Coll., R. Akers et al., Z. Phys. C67 (1995) 389.

[38] A. De Angelis, 'Light Quark Hadrons in Hadronic Z Decays', Proc. EPS HEP Conference, Brussels, 1995.

[39] DELPHI Coll., P. Abreu et al., Z. Phys. C65 (1995) 587.

[40] L3 Coll., Y.J. Pei, 'Studies of meson production in Z decays', CERN Seminar, September 61994 (unpublished).

[41] OPAL Coll., R. Akers et al., Z. Phys. C68 (1995) 1.

[42] ALEPH Coll., D. Buskulic et al., Z. Phys. C69 (1996) 379;

Holger Hepp, Diplomarbeit, HD-IHEP 93-04, Heidelberg (1993).

[43] Review of Particle Properties 1994, Phys. Rev. D50 (1994) 3. 
[44] OPAL Collaboration, M.Z. Akrawy Z. Phys. C47 (1990) 505;

ALEPH Collaboration, D. Decamp et al., Phys. Lett. B273 (1991) 181;

DELPHI Collaboration, A. Abreu et al., Z. Phys. C50 (1991) 185;

OPAL Collaboration, P.D. Acton et al., Z. Phys. C53 (1992) 539.

[45] A. De Angelis, J. Phys. G19 (1993) 1233.

[46] DELPHI Coll. P. Abreu et al., Phys. Lett. B347 (1995) 447.

[47] DELPHI Coll., Contrib. paper eps0556 to EPS HEP Conference, Brussels (1995).

[48] ALEPH Coll., D. Buskulic et al., Phys. Lett. B305 (1993) 415.

[49] ALEPH Coll., Contrib. paper eps0419 to EPS HEP Conference, Brussels (1995).

[50] ALEPH Coll., D. Buskulic et al., CERN-PPE 96-09 (1996).

[51] S. Brandt et al., Phys. Lett. 12 (1964) 57;

E. Fahri, Phys. Rev. Lett. 39 (1977) 1587.

[52] J.D. Bjorken, S. Brodsky, Phys. Rev. D1 (1970) 1416.

[53] R.K. Ellis, D.A. Ross, A.E. Terrano, Nucl. Phys. B178 (1981) 421;

G. Parisi, Phys. Lett. B74 (1978) 65;

J.F. Donohue, F.E. Low, S.Y. Pi, Phys. Rev. D20 (1979) 2759.

[54] L. Clavelli, Phys. Lett. B85 (1979) 111.

[55] S. Catani, G. Turnock, B.R. Webber, Phys. Lett. B295 (1992) 269.

[56] JADE Coll., W. Bartel et al., Z. Phys. C33 (1986) 23;

JADE Coll., S. Bethke et al., Phys. Lett. B213 (1988) 235.

[57] S. Catani et al., Phys. Lett. B269 (1991) 432.

[58] JADE Coll., N. Magnussen et al., Z. Phys. C49 (1991) 29.

[59] C.L. Basham, L.S. Brown, S.D. Ellis, S.T. Love, Phys. Rev. D17 (1978) 2298, Phys. Rev. Lett. 41 (1978) 1585, and Phys. Rev. D19 (1979) 2018.

[60] DELPHI Coll., P. Abreu et al., Z. Phys. C63 (1994) 17;

DELPHI Coll., P. Abreu et al., Phys. Lett. B323 (1994) 242;

DELPHI Coll., P. Abreu et al., Phys. Lett. B355 (1995) 415. 